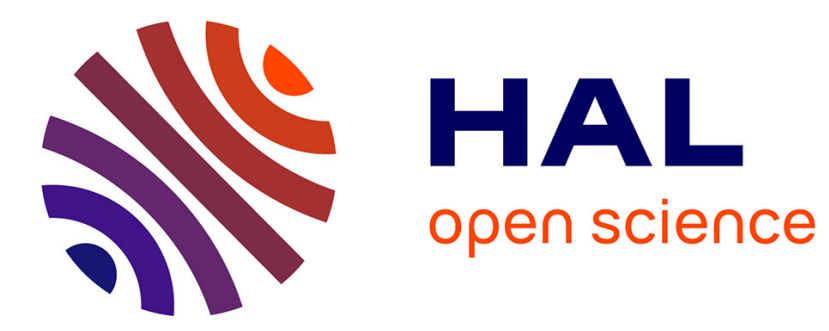

\title{
La place de la sage-femme dans la prise en charge des patientes atteintes de pathologies chroniques
}

\author{
Adeline Voisin
}

\section{To cite this version:}

Adeline Voisin. La place de la sage-femme dans la prise en charge des patientes atteintes de pathologies chroniques. Gynécologie et obstétrique. 2012. dumas-00742906

\section{HAL Id: dumas-00742906 https://dumas.ccsd.cnrs.fr/dumas-00742906}

Submitted on 17 Oct 2012

HAL is a multi-disciplinary open access archive for the deposit and dissemination of scientific research documents, whether they are published or not. The documents may come from teaching and research institutions in France or abroad, or from public or private research centers.
L'archive ouverte pluridisciplinaire HAL, est destinée au dépôt et à la diffusion de documents scientifiques de niveau recherche, publiés ou non, émanant des établissements d'enseignement et de recherche français ou étrangers, des laboratoires publics ou privés. 
CENTRE HOSPITALIER UNIVERSITAIRE DE ROUEN

DEPARTEMENT DES ETUDES DE SAGE-FEMME

\title{
La place de la sage-femme dans la prise en charge des patientes atteintes de pathologies chroniques
}

\author{
Mémoire présenté par \\ Mademoiselle Adeline VOISIN \\ Née le 19 Octobre 1989
}

DIPLOME D'ETAT DE SAGE-FEMME

PROMOTION 2012 


\section{Remerciements}

Dans ce préambule, je tiens à remercier toutes les personnes qui m'ont soutenue dans l'élaboration de mon mémoire :

Madame Deschamps, ma directrice de mémoire, pour tous ses conseils, sa littérature et le temps qu'elle a su m'accorder,

Madame Leguen, sage-femme enseignante, pour sa grande disponibilité, son efficacité et sa guidance,

Toutes les sages-femmes qui ont bien voulu répondre à mon enquête, et les cadres pour leur collaboration,

A tous mes proches, ma famille et mes amis pour leur soutien, leur compréhension et leur aide au cours de la réalisation de ce mémoire et tout au long de mes années d'études,

A toutes les promotions sages-femmes que j'ai connues au cours de ma formation.

MERCI 


\section{Glossaire :}

Ticket modérateur (page 2) : Le ticket modérateur correspond à la partie des dépenses de santé qui reste à la charge du patient après le remboursement de l'Assurance Maladie.

EEG (page 15) : L'électroencéphalogramme (EEG) est l'enregistrement de l'activité électrique du cerveau. Indolore, il consiste en l'application d'électrodes sur le cuir chevelu.

Régime cétogène (page 17) : Le régime cétogène est d'indication exceptionnelle chez certains patients épileptiques -essentiellement des enfants - lorsque le traitement ne diminue pas la sévérité des crises. Composé essentiellement de graisses, pauvre en protéines et glucides, il nécessite une hospitalisation en général d'une semaine, puis un suivi très strict qui consiste notamment en l'analyse régulière des urines (25).

Seuil épileptogène (page 19) : Seuil à partir duquel un stimulus va déclencher une crise chez un patient sujet souffrant d'épilepsie.

Antiépileptiques inducteurs enzymatiques (dans l'ANNEXE VIII) : Au niveau hépatique, les antiépileptiques sont inducteurs de l'izoenzyme 3A4 du cytochrome P450. Ils accélèrent le métabolisme des stéroïdes contraceptifs, ont une action antifolinique et interfèrent avec les métabolismes de la vitamine $\mathrm{K}$ et de la vitamine $\mathrm{D}$. 


\section{Sommaire}

Introduction.

\section{Revue de la littérature :}

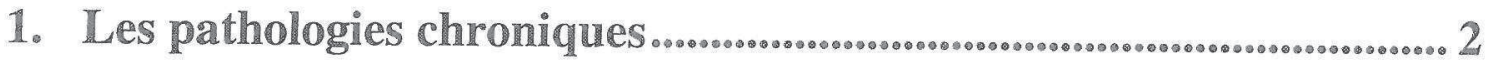

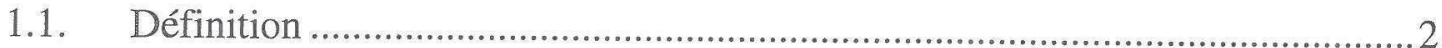

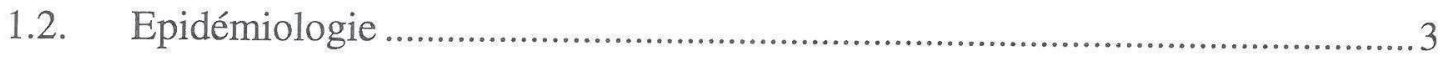

1.3. Les répercussions des pathologies chroniques ............................................. 3

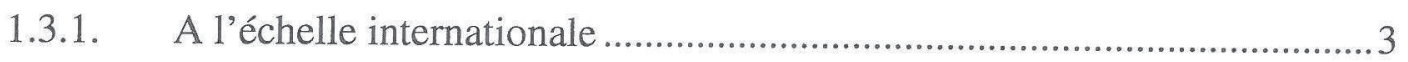

1.3.2. Sur les patients .........................................................................

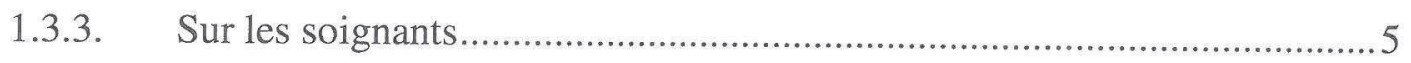

1.4. Pathologies chroniques et grossesse …......................................................

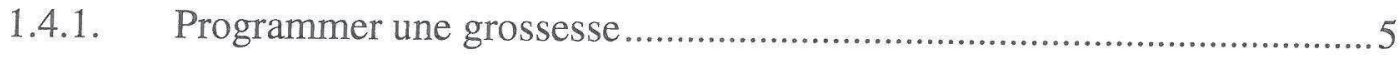

1.4.2. Médicaments et grossesse ................................................................6 6

1.4.3. La prise en charge pluridisciplinaire ................................................. 7

2. L'Education Thérapeutique du Patient ......................................... 8

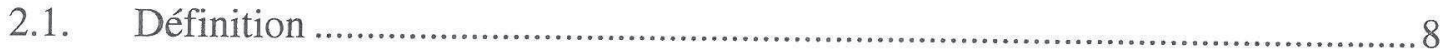

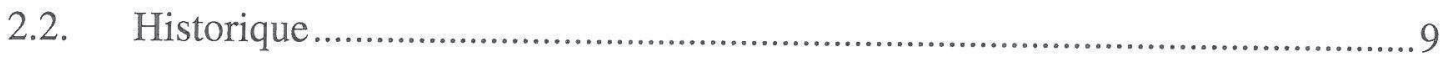

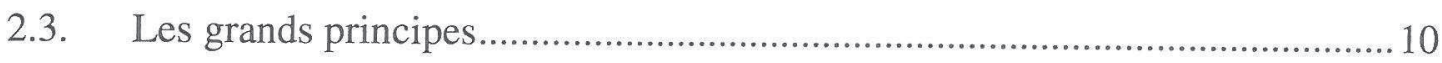

2.3.1. La population bénéficiaire de l'ETP ............................................... 10

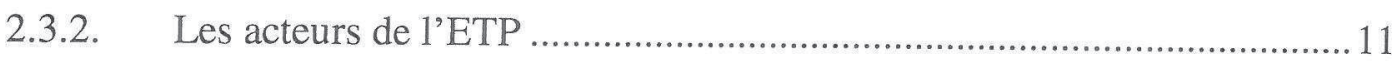

2.3.3. L'organisation de l'éducation thérapeutique du patient......................... 11

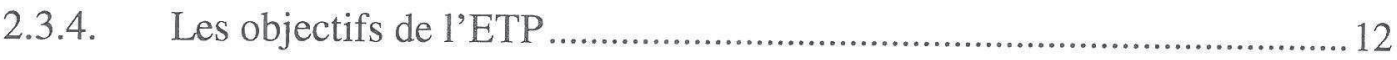

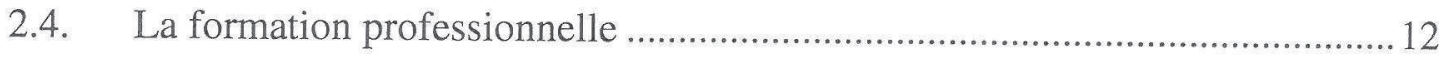

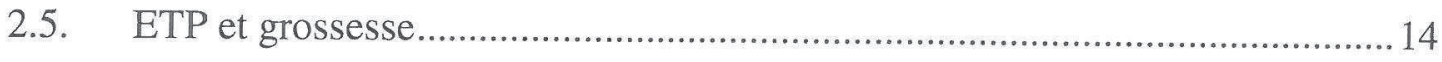

3. Un exemple de prise en charge : l'épilepsie ................................... 14

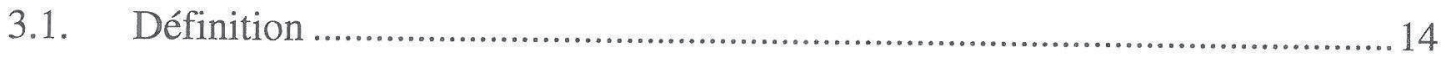

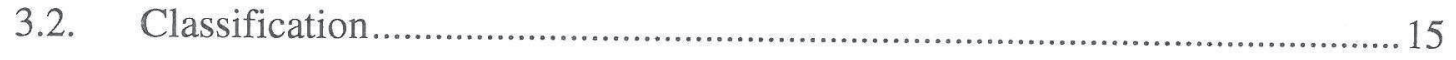

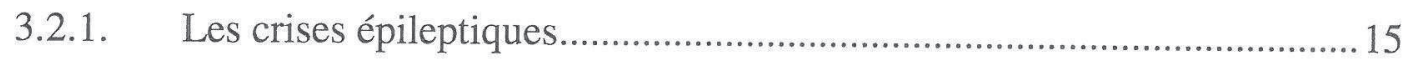

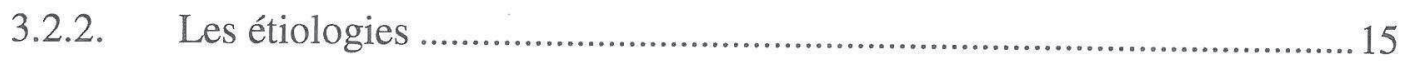

3.3. Diagnostic et prévention ................................................................. 15

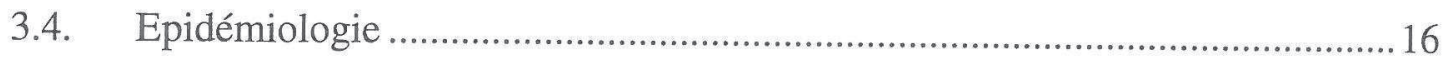

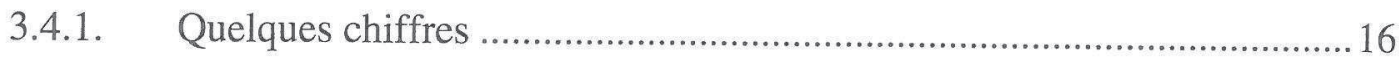

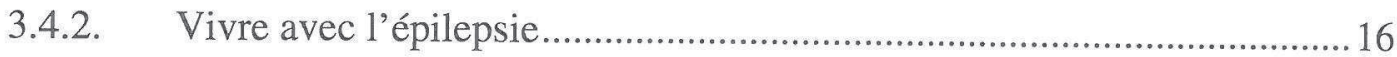




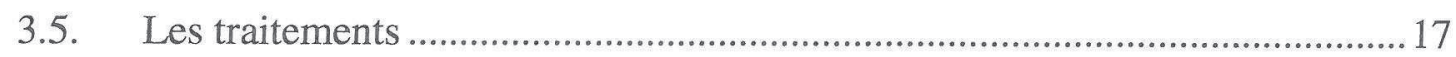

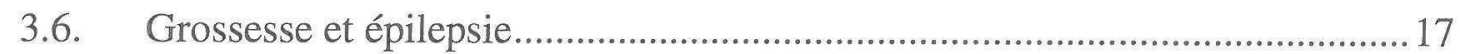

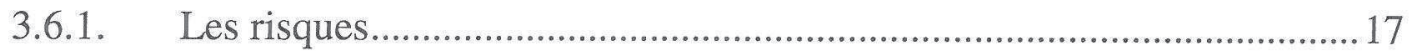

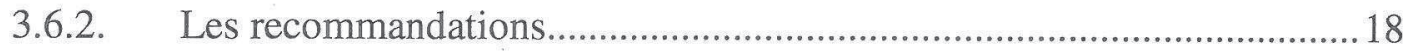

Etude :

1. Méthodologie

1.1 Objectifs de l'étude ...........................................................................20

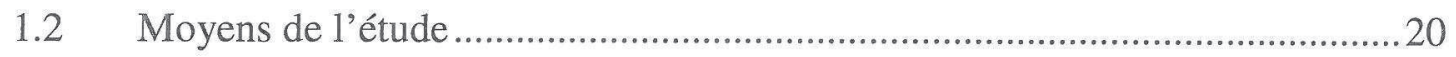

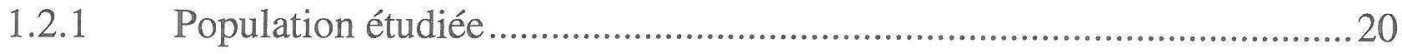

1.2.2 Distribution des questionnaires .......................................................2 21

1.2.3 Les difficultés rencontrées .............................................................22

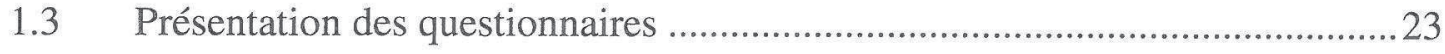

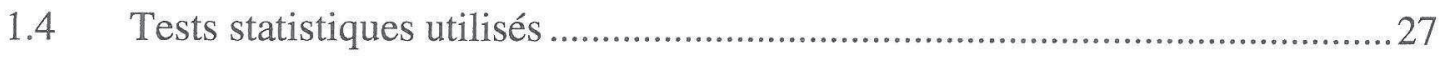

2. Présentation des résultats..................................................... 27

Discussion......................................................49

Conclusion..................................................................60 60

Bibliographie

Sommaire des Annexes

Annexes 


\section{Introduction}

D'après le Code de la Santé Publique, la sage-femme assure, en toute autonomie, le diagnostic, la déclaration et la surveillance de la grossesse normale, le travail et l'accouchement eutociques, ainsi que les soins post-nataux de la mère et de l'enfant. La sage-femme se doit également de dépister la pathologie et d'en référer aux médecins (66).

Selon les statistiques sanitaires mondiales, les pathologies chroniques sont en constante augmentation dans le monde. Elles représentent, aujourd'hui, un problème grave de santé publique.

La grossesse des femmes atteintes de pathologies chroniques, considérée «pathologique », nécessite alors normalement un suivi par un médecin. Pourtant, au cours de notre formation, nous avons pu observer que la sage-femme est tout de même amenée à les prendre en charge.

Notre mémoire ne se veut pas remettre en cause le champ de compétence de la sagefemme. Il se veut un état des lieux de la place qu'elle occupe dans la prise en charge de ces patientes.

Tout d'abord, par une revue de la littérature, nous aborderons successivement trois parties. Dans un premier temps, nous présenterons les pathologies chroniques, leurs définitions et leurs multiples conséquences, notamment en ce qui concerne la grossesse. Nous évoquerons également le concept récent de l'éducation thérapeutique du patient. Enfin, nous avons choisi d'illustrer notre sujet par un exemple de pathologie chronique. L'épilepsie, maladie impressionnante par ses crises et par la difficulté de sa prise en charge, nous a plusieurs fois interpelés au cours de nos stages.

Puis, nous présenterons notre étude réalisée auprès des sages-femmes de Haute Normandie et son analyse, afin de mettre en exergue leurs éventuelles difficultés face aux femmes enceintes malades chroniques.

Pour terminer, nous discuterons alors de la place de la sage-femme et tenterons d'y apporter des solutions. 


\section{Revue de la littérature}




\section{Les pathologies chroniques}

\subsection{Définition}

A contrario de la pathologie aigue, l'Organisation Mondiale de la Santé (OMS) définit la pathologie chronique comme "une maladie qui évolue à long terme, souvent associée à une invalidité ou à la menace de complications sérieuses, et susceptible de réduire la qualité de vie du patient. » (52)

Cette définition est complétée par les notions de :

- " présence d'une cause organique, psychologique ou cognitive ;

- une ancienneté de plusieurs mois ;

- un retentissement de la maladie sur la vie quotidienne : limitation fonctionnelle, des activités, de la participation à la vie sociale ; dépendance vis-à-vis d'un médicament, d'un régime, d'une technologie médicale, d'un appareillage, d'une assistance personnelle ; besoin de soins médicaux ou paramédicaux, d'aide psychologique, d'éducation ou d'adaptation. » (56)

Les pathologies chroniques sont nombreuses et différentes.

Elles sont également qualifiées par l'OMS de «maladies non transmissibles » (MNT). Elles progressent aux côtés des «maladies transmissibles » ou infectieuses (comme le VIH, la tuberculose, les hépatites...). Beaucoup de personnes meurent d'une combinaison des deux. Les systèmes de santé doivent donc s'occuper des deux types en même temps, les maladies infectieuses pouvant par ailleurs devenir chroniques.

Enfin en France, elles sont incluses dans les Affections de Longue Durée (ALD) : «des affections comportant un traitement prolongé et une thérapeutique particulièrement coûteuse, ouvrant droit, pour ceux qui en sont atteints, à l'exonération du ticket modérateur, c'est-à-dire à une prise en charge intégrale de leurs frais de traitement, dans la limite du périmètre remboursable » (51).

Originellement, l'ALD concernait quatre longues maladies. Aujourd'hui, sont exonérés du paiement du ticket modérateur les patients concernés par les maladies recensées dans trois listes (ANNEXE I). 


\subsection{Epidémiologie}

Les maladies chroniques sont en constante progression. Responsables de $63 \%$ des décès en 2008, soit 36 millions sur 57 millions de personnes décédées dans le monde (29\% avaient moins de 60 ans et la moitié étaient des femmes) (52) : elles sont la toute première cause de mortalité mondiale. Près de $80 \%$ des décès (52) surviennent dans les pays à revenu faible ou moyen. Ces affections ne touchent donc pas seulement les pays riches.

Les quatre principalement retrouvées sont :

- les maladies cardio-vasculaires (telles que les crises cardiaques et les accidents vasculaires cérébraux);

- le cancer;

- les maladies respiratoires chroniques (telles que la broncho-pneumopathie chronique obstructive et l'asthme);

- le diabète.

Elles tuent, à elles quatre, trois habitants de la planète sur cinq chaque année.

Dans la catégorie des pathologies chroniques, il ne faut pas oublier les pathologies psychiatriques (dépressions, psychoses, troubles bipolaires...). Celles-ci peuvent effectivement être récurrentes au cours de la vie. Elles nécessitent généralement un traitement à long terme.

En France, les maladies chroniques touchent 15 millions de personnes, atteintes plus ou moins sévèrement, soit $20 \%$ de la population française (56).

\subsection{Les répercussions des pathologies chroniques}

\subsubsection{A l'échelle internationalle}

Ces chiffres alarmants constituent un véritable problème de santé publique.

En effet, en Avril 2011, à Moscou, un Forum mondial de l'OMS sur les maladies non transmissibles a réuni près de 300 personnalités des secteurs civil et privé, des milieux universitaires. A cette occasion fut édité le Rapport sur la situation mondiale des maladies non transmissibles (MNT) dans 193 pays. (52)

Pour le Directeur général de l'OMS, le Dr Margaret Chan, «l'augmentation des maladies chroniques non transmissibles représente un énorme défi. Pour certains pays, 
il n'est pas exagéré de décrire la situation comme une catastrophe imminente, une catastrophe pour la santé, pour la société et surtout pour les économies nationales.»:

- En France, plus de 7 millions d'assurés sont atteints d'une Affection de Longue Durée (ALD). Ce nombre a augmenté de $60 \%$ en 10 ans. Ce qui implique chaque année 80 milliards d'euros consacrés à la prise en charge des ALD et près de $65 \%$ des remboursements de 1'assurance Maladie (56).

- Près de $30 \%$ des personnes qui meurent de maladies non transmissibles dans les pays à revenu faible ou moyen sont âgées de moins de 60 ans (52) et sont dans leurs années les plus productives. Ces «décès prématurés », en grande partie évitables, contribuent, comme dans un cercle vicieux, à la pauvreté.

Si les principaux facteurs de risque de maladies chroniques (le tabagisme, la sédentarité, l'usage nocif de l'alcool, l'alimentation déséquilibrée, le cholestérol,...) étaient éliminés, près des trois quarts des cardiopathies, des accidents vasculaires cérébraux et des cas de diabète de type 2 pourraient être évités ainsi que $40 \%$ des cancers.

Le Dr.Chan ajoute: «faute de mesures, on prévoit que l'épidémie des MNT tuera 52 millions de personnes chaque année d'ici $2030 »$.

Ce nouveau rapport est donc un élément clé du plan d'action 2008-2013 pour la mise en œuvre de la Stratégie mondiale OMS de lutte contre les maladies non transmissibles. Il a d'ailleurs été complété par la réunion de haut niveau des Nations Unies sur la prévention et la maîtrise des maladies non transmissibles, les 19 et 20 septembre 2011 à New York. (53)

\subsubsection{Sur les patients}

Un patient diagnostiqué malade chronique subit une rupture existentielle.

Cette maladie ni choisie, ni désirée peut se manifester par des douleurs physiques, avec des menaces parfois vitales, ou bien être silencieuse mais dans tous les cas, elle signe une perte de l'intégrité et a des répercussions sur tous les plans (la vie sociale et professionnelle, mais aussi la vie affective et familiale).

D'un point de vue psychologique, être atteint d'une pathologie chronique, c'est-àdire non guérissable, implique «la perte de son état de santé antérieur».

Le porteur de la maladie doit opérer une sorte de deuil. 
Ainsi, en se basant sur le modèle du travail du deuil de Freud, on trouve deux processus différents face à la maladie : l'acceptation ou la résignation. (ANNEXE II). Dans les deux cas, le choc de l'annonce de la pathologie provoque une stupeur, phénomène normal. Le modèle théorique expose :

- dans le premier cas, l'individu passe par une étape de révolte puis de tristesse profonde (la nostalgie de ce qui est perdu) et enfin il cède devant l'évidence du fait.

- dans le processus de résignation ou de distanciation, il est dans le déni, d'où la difficulté des thérapeutiques, et sombre dans la dépression.

Dans la réalité, les différentes phases s'entrecroisent. Au final, ce travail de deuil lui permet, avec de l'aide, d'admettre un jour la réalité de son état de santé.

D’un point de vue médico-social, à long terme, les pathologies chroniques entrainent déficience, incapacité et désavantage. Elles nécessitent une surveillance plus accrue au cours de la vie: grossesse, anesthésie, opération... et de nombreuses contraintes: traitements à vie, modifications des habitudes de vie (hygiène, alimentation)...

\subsubsection{Sur les soignants}

En règle générale, avec les progrès en médecine, le soignant a l'impression de pouvoir tout réparer, tout guérir, en particulier avec les pathologies aiguës (courte durée du traitement, nombre limité d'intervenants, passivité du patient et guérison). Avec les pathologies chroniques, la prise en charge a des caractéristiques inverses : traitement de longue durée, souvent la vie entière du patient, des interventions nombreuses et variées de professionnels différents. Le personnel soignant et notamment les médecins se doivent au mieux de freiner la dégradation ou la survenue de complications, sans espérer la guérison. Ils ressentent une frustration : «Autant dur pour le soignant que pour le patient de renoncer à l'idée de pouvoir guérir » (2)

\subsection{Pathologies chroniques et grossesse}

La femme enceinte atteinte d'une pathologie chronique a une grossesse considérée plus à risque que la femme sans problème de santé.

\subsubsection{Programmer une grossesse}

L'abrogation de la consultation prénuptiale en 2007, considérée inefficace compte tenu de la naissance hors mariage d'un enfant sur deux, aurait dû entrainer l'essor de la consultation pré-conceptionnelle. 
En effet, pour de nombreux obstétriciens, celle-ci devrait être proposée à l'ensemble des futures mères afin que des situations dangereuses puissent être évitées dès le début de grossesse, des mesures hygiéno-diététiques mises en place, la prise en charge des addictions anticipée.

En France, elle n’est proposée qu'aux femmes présentant une pathologie médicale susceptible de mettre la santé de leur fotus et la leur en danger, notamment dans le cadre des pathologies chroniques.

Au cours de cette consultation, le spécialiste de la pathologie se doit de programmer une surveillance en alternance avec l'équipe obstétricale. Il peut supplémenter les patientes, prescrire des examens complémentaires nécessaires avant la conception.

Surtout, il est amené à adapter le traitement (dosage, molécule...) en fonction de l'arrêt d'une contraception par exemple, ou encore pour éviter la survenue de fausses couches précoces ou de malformations fœtales.

\subsubsection{Médicaments et grossesse}

Tous les médicaments administrés à une femme enceinte sont considérés comme potentiellement dangereux pour le foetus.

La femme enceinte présente un terrain particulier pour la pharmacocinétique d'un médicament du fait des interactions entre son corps, le placenta et le foetus.

- Au niveau de la résorption du médicament

L'augmentation de la progestérone entraine un ralentissement du tractus gastroduodénal et donc retarde le passage du principe actif dans le duodénum. La résorption en est retardée.

Le $\mathrm{pH}$ gastrique augmente au $1^{\mathrm{er}}$ trimestre, entrainant une fraction libre (active) plus importante du médicament.

- Au niveau de la distribution du médicament

Pour être distribué aux différents organes, le principe actif doit être lié aux protéines plasmatiques. Or, pendant la grossesse, s'ajoutent des nouveaux compartiments : l'utérus, le placenta, le foetus. Ceux-ci entrainent des variations du volume plasmatique.

La fraction libre (non liée) du principe actif peut se trouver augmentée et provoquer un surdosage. Lorsque plusieurs traitements sont administrés, on observe des interactions médicamenteuses entrainant une compétition des différentes fractions libres à se fixer aux protéines. 
- Au niveau de l'élimination du médicament

Le médicament est éliminé par l'excrétion rénale et le métabolisme hépatique.

Dès le début de grossesse, les reins ont une activité accrue (augmentation du flux sanguin rénal, de la filtration glomérulaire et de la clairance de la créatinine). Il en découle une augmentation de l'excrétion des médicaments administrés et donc une concentration plasmatique diminuée.

- Le placenta

Le placenta est une véritable «passoire» plutôt que barrière. Par différents mécanismes (diffusion simple, diffusion passive, transport actif, pinocytose), la plupart des médicaments contenus dans la circulation maternelle passe le placenta.

La problématique restant à savoir : est-ce qu'ils passent dans la circulation fotale ?

Entrainent-ils des conséquences chez le fotus ? En combien de temps les éliminent-ils ? Un grand nombre de ces réponses a été étudié et des recommandations ont été faites par le CRAT (centre de référence sur les agents tératogènes).

D'où la nécessité de réaliser des contrôles sérologiques de la concentration plasmatique de certains traitements de pathologies chroniques pendant la grossesse (exemple notamment des traitements antiépileptiques). Le but recherché est d'adapter leur posologie pour qu'ils restent efficaces en traitant la pathologie tout en évitant le risque de tératogénèse.

\subsubsection{La prise en charge pluridisciplinaire}

Pour les femmes atteintes d'une pathologie chronique, le maintien de leur santé et le suivi de leur pathologie doit rester une priorité pendant la grossesse. C'est pourquoi le spécialiste de 1' « organe » doit être consulté régulièrement.

La grossesse considérée «pathologique » nécessite un suivi régulier par un médecin (généraliste, obstétricien ou gynécologue). Elle s'inscrit, en effet, dans la limite du champ de compétence de la sage-femme et ne peut faire l'objet d'un suivi exclusif par celle-ci.

D'autres intervenants, tout aussi importants, interviennent du fait des complications qu'entrainent ces pathologies : les anesthésistes, les pédiatres, les échographistes... 
Depuis quelques années, une nouvelle discipline évolue dans le cadre du suivi des pathologies chroniques afin d'en améliorer la prise en charge. On parle de l'éducation thérapeutique du patient.

\section{L'Education Thérapeutique du Patient}

\subsection{Définition}

Il y a quelques années, on parlait simplement d'éducation du malade.

Aujourd'hui, on fait la différence entre deux notions: éducation thérapeutique et éducation pour la santé.

L'éducation pour la santé concerne la population générale, avec des programmes tels que des mesures de vie saine, l'alimentation équilibrée, la prévention du sida, de l'alcoolisme, du tabagisme...

L'éducation thérapeutique du patient (ETP), elle, est un concept nouveau apparu depuis quelques années et qui ne peut être dispensé que par des professionnels de soins.

Le terme a été défini par l'OMS (52) pour éviter les confusions avec «l'éducation pour la santé » et la simple « information des patients».

L'éducation thérapeutique «vise à aider les patients à acquérir ou maintenir les compétences dont ils ont besoin pour gérer au mieux leur vie avec une maladie chronique. Elle fait partie intégrante et de façon permanente de la prise en charge du patient. Elle comprend des activités organisées, y compris un soutien psychosocial, conçues pour rendre les patients conscients et informés de leur maladie, des soins, de l'organisation et des procédures hospitalières, et des comportements liés à la santé et à la maladie. Ceci a pour but de les aider, ainsi que leurs familles, à comprendre leur maladie et leur traitement, à collaborer ensemble et à assumer leurs responsabilités dans leur propre prise en charge, dans le but de les aider à maintenir et améliorer leur qualité de vie. »

Le patient devient acteur. L'ETP s'appuie sur son vécu et son expérience antérieure. Elle est multi professionnelle, interdisciplinaire et intersectorielle.

Cette activité spécifique n'existe pas dans la formation médicale initiale (médecins, sages-femmes...). En revanche, il s'agit d'une unité d'enseignement dans les études d'infirmiers.

Le terme «d'éducation des patients » vient des anglo-saxons. En France, les professionnels aiment souvent mieux parler de «formation thérapeutique » : cela donne éthiquement moins la notion d'autorité sur autrui. Aux Etats-Unis, le « disease managment » est plus un modèle de rentabilité, plus pragmatique (2). 
Cependant, l'ETP n'est qu'une composante des stratégies d'accompagnement du malade au cours des années. Elle s'inscrit dans le cadre de la prévention tertiaire.

\subsection{Historique (2)}

Dans l'Antiquité déjà, Hippocrate proclamait que le médecin doit collaborer avec le malade. La personne atteinte ne doit plus «subir » la maladie mais assumer une part active dans le processus thérapeutique.

La France a été lente à la mise en place d'éducation pour la santé notamment du fait de ses traditions hygiénistes strictes et de sa formation initiale des médecins centrée sur les soins curatifs. Depuis 15 ans seulement, les responsables politiques et institutionnels prennent conscience de la nécessité de l'éducation des citoyens à la santé, du fait de l'évolution de l'état de santé de la population et de la crise que traverse notre système de protection sociale.

Le diabète a été la pathologie «charnière » de $1^{\prime} E T P$ avec la création des premières associations de patients en 1956 «Aide aux jeunes diabétiques », de La Maison du diabète en 1987 et des associations de professionnels en 1989 : « Diabetes Education Study Group ».

D’une manière plus générale, en 1994, se mettent en place les premières formations pour les professionnels, en particulier les infirmiers, par le biais des Ipcem (Instituts de perfectionnement en communication et éducation médicales) et s'appuyant sur la Charte d'Ottawa publiée en 1986 par l'OMS recommandant aux services de santé de se centrer «sur la totalité des besoins de l'individu considéré dans son intégralité ».

En 1995, le Comité Français d'Education pour la Santé (CFES) encourage et soutient l'implication des professionnels de santé, notamment les médecins généralistes et les pharmaciens d'officine dans l'éducation des patients.

A partir de 1998, La Direction Générale de la Santé (DGS) met en place un groupe de travail multidisciplinaire en accord avec la Direction des hôpitaux et la sécurité sociale pour enquêter sur les pratiques éducatives dans les établissements de santé français.

L'apparition de l'éducation thérapeutique dans les textes réglementaires commence alors en 1999 :

- l'Agence Nationale d'Accréditation et d'Evaluation en Santé (ANAES) mentionne l'éducation du patient dans son référentiel OPC5 (Organisation et 
prise en charge): tous les quatre ans, les établissements de santé doivent s'assurer de leurs actions en matière d'éducation du patient et les améliorer si besoin.

- En 2001, le plan national d'éducation pour la santé présenté par Bernard Kouchner vise à développer, généraliser et valoriser l'ETP.

- Entre 2001 et 2002 s'ajoutent d'autres plans : le programme national nutrition santé et des programmes notamment sur le diabète de type 2, 1'asthme et les risques cardio-vasculaires. Chacun comprend un volet relatif à l'ETP.

- Le 4 Mars 2002, la loi relative au code de la santé publique déclare que le patient peut participer aux décisions thérapeutiques qui le concernent.

Avec la création de 1'INPES (Institut national de prévention et d'éducation pour la santé), la première circulaire sur l'ETP est publiée.

- L'article 84 de la loi Hôpital, Patients, Santé et Territoires de 2009 inscrit l'éducation thérapeutique dans le code de Santé publique. Elle s'inscrit dans le parcours de soins.

La HAS et l'INPES ont donc investi des programmes, des plans et guides disponibles pour tous, conformes à un cahier des charges national (23). Les programmes d'ETP sont mis en œuvre au niveau local après autorisation des Agences Régionales de Santé (ARS). Une grille d'aide à l'évaluation élaborée par l'HAS permet de vérifier la qualité du programme dans le but de son autorisation.

Chaque ARS bénéficie d'une somme issue de l'enveloppe des Missions d'Intérêt général pour financer les programmes d'ETP (66) (67).

\subsection{Les grands principes}

\subsubsection{La population bénéficiaire de $l^{9} \mathrm{ETP}$}

L'ETP est proposée à une personne (enfant, adolescent ou adulte) atteinte d'une maladie chronique quels que soient son âge, le type, le stade et l'évolution de sa maladie et à son entourage, s'il le souhaite (parents d'enfants ayant une maladie chronique, conjoint ou compagnon, fratrie, enfants de parents malades, personne de confiance...). Le patient choisit de participer ou non à l'ETP et d'impliquer ou non ses proches dans l'aide à la gestion de sa maladie. 


\subsubsection{Les acteurs de l’'ETP}

Tout professionnel de santé, selon la liste du Code de santé publique, et formé à l'ETP peut intervenir ainsi que d'autres professionnels : psychologue, travailleur social, éducateur en activité physique adaptée, pédagogue de la santé, etc.

Il nécessite entre eux une bonne coordination et une transmission d'informations.

\subsubsection{L'organisation de l'éducation thérapeutique du patient}

Un programme personnalisé en éducation thérapeutique se compose de plusieurs étapes :

- Formuler un diagnostic éducatif : la première étape consiste à identifier les besoins du patient puis à définir avec lui les compétences (ANNEXE III) qu'il souhaite acquérir (séries d'entretiens pour définir ce qu'a le patient, ce que fait le patient, ce que sait le patient, ce qu'il est et enfin ses projets).

- Planifier et mettre en ouvre les séances d'éducation thérapeutique avec différentes méthodes pédagogiques variées: collectives ou individuelles, pour adulte ou pour enfant, des patients tuteurs, des associations de patients... Elles facilitent les interactions et les médiations avec le patient et ses proches et permettent l'acquisition de compétences.

Le programme d'ETP mis en place doit également respecter et s'intégrer à la stratégie thérapeutique. Il se déroule en hôpital et/ou en libéral pour assurer un continuum.

- Identifier des situations conduisant à proposer au patient des interventions spécifiques : la reprise éducative.

De nouveaux évènements dans la vie (la grossesse pour les femmes), des situations de vulnérabilité psychologique et sociale, des co-morbidités, des troubles psychiques (stress, anxiété, troubles du sommeil, dépression), des addictions peuvent survenir au fil du temps. Une orientation vers un professionnel du champ médico-social peut être nécessaire.

Les professionnels de santé doivent donc être attentifs à ces situations et aux signes d'alerte à n'importe quelle étape du programme.

Leurs prises en charge peuvent amener à une modification des besoins du patient. 
- L'évaluation individuelle des compétences acquises et du déroulement du programme: il s'agit de l'évaluation par le patient de ce qu'on lui a enseigné. Le but est d'évaluer tous les domaines entrant dans le cadre de 1'ETP (cognitif, sensori-moteur, psycho-affectif ). L'évaluation indique ainsi au patient et aux professionnels de santé ce que le patient sait, ce qu'il a compris, ce qu'il sait faire et appliquer, ce qu'il lui reste éventuellement à acquérir.

L'évaluation individuelle de l'éducation thérapeutique est proposée au minimum à la fin de chaque programme mais aussi à tout moment de la prise en charge si le professionnel de santé le juge nécessaire ou si le patient la sollicite.

\subsubsection{Les objectifs de $1^{9} E T P$}

Le patient doit être capable de gérer sa pathologie afin de diminuer sa dépendance et intégrer son handicap à sa vie quotidienne.

A. LACROIX, et J.P. ASSAL, auteurs de nombreux ouvrages sur ce concept (2), précisent que pour y parvenir, l'ETP aide le patient à acquérir des étapes intermédiaires :

1) Exprimer ses besoins

2) Comprendre (son corps, sa maladie...)

3) Repérer les signes d'alerte

4) Faire face à une crise, prendre une décision

5) Ajuster sa thérapeutique, aménager son environnement

6) Pratiquer des techniques, 1'auto-soin

7) Adapter la thérapeutique à un autre contexte de vie (comme la grossesse)

8) Faire valloir ses droits.

\subsection{La formation professionnelle}

Les maladies chroniques obligent le soignant à se centrer sur le porteur de la maladie, le patient, qui vit toute l'année, jour après jour avec sa souffrance.

Passer du stade de l'aigu à celui de l'accompagnement de longue durée implique que le soignant fasse une conversion dans sa manière de traiter la maladie : il est formé aux techniques de communication et aux techniques pédagogiques, au travail en équipe et à la coordination des actions.

Cette formation est un moyen de lutte contre la non-observance du patient. 
Les intervenants en ETP doivent justifier de compétences en éducation thérapeutique (relationnelles, pédagogiques et d'animation, méthodologiques et organisationnelles, biomédicales et de soins).

Ces compétences doivent s'acquérir par une formation d'une durée minimale obligatoire de 40 heures d'enseignements théoriques et pratiques (68) (69), soit dans le cadre de la formation initiale (exemple de la formation des infirmiers : en $2^{\text {ème }}$ année, l'ETP fait partie d'une unité d'enseignement d'un peu plus de 50h de cours magistraux, Travaux Pratiques et Travaux Dirigés), soit dans le cadre de la formation continue à deux niveaux :

- Le niveau 1: Diplôme Universitaire (DU) ou Interuniversitaire (DIU). Il s'agit d'une formation de base pour les professionnels en charge d'éduquer les patients.

Qualifiée de courte (50 à 120 heures sur une année), elle est délivrée par des organismes de formation publics comme l'IPCEM (Institut de perfectionnement en communication et éducation médicales), privés ou associatifs et des universités.

Elle est plus souvent choisie par les professionnels de santé déjà formés au cours de leur cursus (infirmiers, kinésithérapeutes,...).

Elle respecte le cahier des charges et les recommandations fixés par l'HAS.

- Le niveau 2 ou formation longue (plus de 400 heures) : le master. Il permet à terme d'acquérir des fonctions de responsabilités dans la coordination institutionnelle, l'encadrement, l'évaluation, de gérer un programme d'ETP.

A l'université de Rouen est délivré le Master 2 Professionnel Ingénierie de l'éducation thérapeutique.

Ces deux types de formations s'adressent aux :

- Diplômés : médecin, infirmier, diététicien, kinésithérapeute, podologue, ergothérapeute, pharmacien, psychologue, médecin, orthophoniste, sagefemme...

- Public pluri-professionnel et pluridisciplinaire: les professionnels de santé hospitaliers, libéraux ou travaillant dans une autre institution ou dans un réseau de soin, concernés par l'éducation des patients (éducateurs sportifs, assistants sociaux des services de soin...).

- Patient-expert 
L'ensemble des professions de santé devrait acquérir des compétences minimales en formation initiale. Les récentes enquêtes de l'INPES à ce sujet ont montré que des progrès importants restent à faire.

En première ligne, on retrouve le plus souvent les infirmier (ère)s. Aujourd'hui, plus de médecins l'investissent.

\subsection{ETP et grossesse}

L'OMS a défini une liste de soixante maladies chroniques ou états du malade, où l'efficacité du traitement est déterminée par le degré de formation thérapeutique du malade. On y retrouve en deuxième position : «Affections liées au cycle de la vie (ex : grossesse, enfance, vieillesse) ».

Ainsi, la grossesse est déjà considérée comme une affection à courte durée nécessitant une éducation thérapeutique. On devine donc l'intérêt que devrait avoir l'ETP lorsqu'une femme est atteinte d'une pathologie chronique et enceinte. Pourtant, il s'agit d'une discipline peu connue par les sages-femmes et dans laquelle on la retrouve rarement.

Parmi les pathologies chroniques, nous verrons dans cette troisième partie 1'exemple de l'épilepsie.

\section{Un exemple de prise en charge : l'épilepsie}

\subsection{Définition}

Il est difficile de parler de l'épilepsie au singulier mais plutôt « des » épilepsies. Cette pathologie a en effet différentes étiologies et se traduit par des expressions cliniques et des évolutions multiples.

Le mot épilepsie vient du grec « epilepsia » : attaquer, saisir par surprise.

Il s'agit d'une affection chronique d'origine cérébrale se caractérisant par la répétition de crises épileptiques chez un même sujet.

Ces crises se traduisent par des manifestations paroxystiques motrices, sensitives, sensorielles ou psychiques, accompagnées ou non d'une altération de la conscience. Elles sont le résultat de décharges électriques excessives et hypersynchrones d'une population plus ou moins étendue de neurones du cortex cérébral.

Une crise unique ne signe pas l'épilepsie (chacun, jusqu'à 10\% de la population mondiale, peut en avoir une au cours de sa vie). Elle se définit par la survenue spontanée d'au moins deux crises. 


\subsection{Classification}

Les épilepsies sont classées en fonction des types de crises mais aussi en fonction des étiologies retrouvées.

\subsubsection{Les crises épilleptiques}

On distingue les crises généralisées où la décharge épileptique intéresse d'emblée tout le cerveau de façon bilatérale, synchrone et symétrique, et les crises partielles où la décharge a un début focal, unilatéral. Une crise partielle peut se généraliser secondairement.

La situation d'urgence reste l'état de mal épileptique: crises récurrentes sans intervalle de conscience normale ou crise unique prolongée (supérieure à 5 minutes) (ANNEXE IV).

\subsubsection{Les étiologies}

On retrouve les épilepsies d'origine symptomatique (conséquence d'une affection définie : accidents vasculaires cérébraux, tumeurs, alcoolisme, traumatismes, troubles métaboliques...), idiopathique (des crises à symptomatologie clinique précise et dont l'origine génétique est probable) et cryptogénique dans deux tiers des cas (les épilepsies n'entrant dans aucune des deux catégories précédentes, sans cause connue).

\subsection{Diagnostic et prévention}

Les manifestations cliniques de l'épilepsie et la fréquence des crises étant très diverses, le diagnostic repose essentiellement sur une anamnèse détaillée et une observation de la crise afin de faire la distinction d'emblée entre crise épileptique et crises psychogènes (crise de tétanie, attaque de panique, crises psychiques, crises neurogènes), syncopes (perte de connaissance avec plus ou moins perte du tonus postural) ou d'autres étiologies (aura migraineuse, accident ischémique transitoire, ictus amnésique, crises hémodynamiques, crises métaboliques...).

C'est souvent à l'occasion d'une crise généralisée inaugurale que le diagnostic est posé. La confirmation du diagnostic se fait par électroencéphalographie. 
La prévention est impossible pour l'épilepsie idiopathique. En revanche, on peut prendre des mesures pour éviter les causes connues d'épilepsie secondaire: la prévention des traumatismes de la tête pour éviter l'épilepsie post-traumatique, la qualité des soins périnataux pour réduire le nombre des nouveaux cas d'épilepsie dus à des traumatismes à la naissance, les préventions contre la fièvre chez l'enfant...

\subsection{Epidémiologie}

\subsubsection{Quelques chiffres}

En 2009, 1’Organisation Mondiale de la Santé estimait que 4 à 10 personnes pour 1000 souffraient d'épilepsie évolutive dans la population générale soit environ 50 millions de personnes: c'est-à-dire autant que pour le SIDA (52). Dans les pays développés, le nombre annuel des nouveaux cas se situe entre 40 et 70 pour 100000 habitants (52). Dans les pays en développement, les chiffres sont fréquemment deux fois plus élevés en raison toujours du manque de soins. En France, environ 500000 personnes sont répertoriées comme épileptiques dont environ 300000 sont sous traitement. Chaque année, on recense plus de 20000 nouveaux cas (42).

\subsubsection{Vivre avec l'épilepsie}

L'épilepsie augmente de deux à trois fois environ le risque de décès prématuré par rapport au reste de la population.

Il s'agit d'une des affections les plus anciennement connues de l'humanité. Elle a suscité pendant des siècles la crainte, l'incompréhension, les discriminations et la stigmatisation sociale. Cela continue de nos jours dans de nombreux pays.

Cette pathologie fait peur par ses crises mais le handicap généré ne se limite pas à cela, la maladie pouvant également entraver le fonctionnement de l'ensemble du cerveau (mémoire, langage, praxies, ou moins spécifiquement les fonctions neurocognitives: concentration, fatigabilité, apprentissage, hyperactivité). C'est pourquoi contrairement aux autres malades chroniques (cancer, asthme, diabète...), les épileptiques ont des difficultés d'insertion scolaire, professionnelle, et l'aptitude à la conduite automobile ne peut être attestée que par un neurologue. Les épileptiques se doivent de tout planifier au cours de leur vie : anesthésie, grossesse... 
L'OMS et ses partenaires reconnaissent qu'il s'agit d'un grand problème de santé publique. Ils mènent une campagne mondiale pour informer et faire mieux connaître l'épilepsie, ainsi que pour renforcer l'amélioration des soins (19).

\subsection{Les traitements}

Les crises peuvent être maîtrisées dans 60 à $70 \%$ des cas grâce à des médicaments antiépileptiques (AE) (ANNEXE V).

La plupart des $\mathrm{AE}$ peuvent entrainer somnolence, troubles de la concentration et ataxie, à doses élevées. Beaucoup sont inducteurs enzymatiques. Dans 30\% des cas, 1'épilepsie est dite "pharmaco-résistante". D'autres solutions sont alors à envisager telles que l'intervention neurochirurgicale si la lésion est bien repérée ou le régime cétogène.

Un suivi par EEG est nécessaire tous les deux ans si l'état est stable, plus fréquent au besoin.

\subsection{Grossesse et épillepsie}

On compte près de 100000 femmes françaises épileptiques en âge de procréer. Ce qui fait de l'épilepsie la maladie neurologique chronique la plus fréquemment retrouvée chez la femme enceinte (52).

L'association « épilepsie et grossesse » n'est donc pas exceptionnelle $(0.5 \%$ des grossesses) (43). 5000 grossesses environ en France sont menées à terme chaque année par ces femmes (4).

\subsubsection{Les risques}

Dans la littérature et notamment dans des études de qualité (15) (20), les risques retrouvés sont de deux ordres :

a) un retentissement de la grossesse sur l'épilepsie par une modification de la fréquence des crises épileptiques.

Le pourcentage de patientes dont la fréquence des crises restait inchangée variait de 54 à $80 \%$. Une diminution de la fréquence des crises se retrouvait dans 3 à $24 \%$ des cas et une augmentation dans 14 à 32\%, souvent, malgré la poursuite de prise des antiépileptiques (cf partie I, 4.2).

Une absence de crise pendant au moins 9 mois avant la grossesse est probablement associée à une probabilité élevée (84-92\%) de ne pas en faire pendant la grossesse. 
Une des principales causes du déséquilibre de l'épilepsie pendant la grossesse reste la non compliance au traitement.

b) Réciproquement, une influence de l'épilepsie et notamment des traitements antiépileptiques sur la grossesse: les risques tératogènes sont multipliés par 2 voire 3 par rapport à une grossesse sans traitement. Le plus important facteur de risque de malformations est la polythérapie. (ANNEXE VI)

Cependant, les crises épileptiques non maitrisées, en particulier généralisées tonicocloniques ou l'état de mal épileptique, sont dangereuses pendant la grossesse et l'abandon de prise d'antiépileptiques posent un risque plus élevé de mortalité pour la mère et pour le fœtus que les effets secondaires possibles du médicament.

Il n'est pas observé plus de complications obstétricales spécifiques par rapport à une grossesse normale :

- Ces femmes ont un risque modérément accru d'accouchement par césarienne.

- Aucune preuve solide d'une augmentation des risques de pré-éclampsie, d'hypertension gravidique et d'avortement spontané.

- Pas de risque accru de menace d'accouchement prématuré ni d'accouchement prématuré. Toutefois, 1'association tabac et/ou alcool est beaucoup plus à risque par rapport aux femmes non épileptiques.

- Un risque modérément accru de complications hémorragiques.

Les risques foetaux et néonataux sont de plusieurs ordres :

- Malformations congénitales

- Retard de croissance intra-utérin avec les carbamazépines (Tégrétol )

- Anomalie de la minéralisation osseuse

- Morbi-mortalité périnatale suite à une ou plusieurs crises maternelles majeures (bradycardie, acidose, hypoxo-ischémie), prématurité

- Syndrome hémorragique par déficit en vitamine K

- Imprégnation par les traitements anti-épileptiques: syndrome de sevrage ou léthargie (hypotonie, mauvaise succion, dépression respiratoire possible)

- Risque de développement d'une épilepsie dépendant de l'étiologie maternelle ou intégrant un syndrome génétique estimé entre 2 et $5 \%$ (5)

- Risque de troubles cognitifs si l'acide valprö̈que est utilisé pendant la grossesse

- Rarement hypotrophie, anémie macrocytaire, hypocalcémie 


\subsubsection{Les recommandations}

Les dernières recommandations du CNGOF (Collège National des Gynécologues et Obstétriciens Français) retrouvées à ce sujet remontent à 2007. Après une revue exhaustive de la littérature, voici en ANNEXE VII les recommandations générales souhaitables d'être appliquées.

Il est évident qu'il faut connaître les gestes d'urgence à réaliser en temps de crise (ANNEXE IX).

Certaines études (34) (40) ont également proposé des conduites à tenir intéressantes dans leurs conclusions :

$>$ Déclarer de façon systématique à la pharmacovigilance toutes les patientes exposées à des traitements antiépileptiques car en règle général, ne sont déclarées que celles dont le foetus présente une malformation. Ce qui implique encore des biais.

$>$ En cas d'antécédent d'épilepsie non «active », rester attentif au risque de survenu de crises en raison de la diminution du seuil épileptogène au cours de la grossesse.

Ces nombreuses recommandations montrent à quel point les pathologies chroniques, ici l'épilepsie, nécessitent une prise en charge bien plus complexe que pour des femmes bien portantes ayant une grossesse physiologique.

Un programme d'ETP proposé à une femme atteinte d'une pathologie chronique en âge de procréer semble un excellent moyen de l'informer, l'éduquer et la rassurer. Les professionnels de la naissance peuvent tous y trouver un rôle. 


\section{ETUDE}




\section{Méthodologie}

\subsection{Objectifs de l'étude}

Les sages-femmes sont les interlocutrices principales de la femme et du couple au cours de la grossesse. Elles sont autonomes dans la physiologie mais leur champ de compétence se trouve restreint en cas de pathologie.

Or, comme nous l'avons vu, les pathologies chroniques sont en constante augmentation. Les sages-femmes sont souvent amenées à rencontrer des patientes porteuses de ces maladies quelque soit leur lieu d'exercice.

Ainsi, cette étude se veut être un état des lieux sur la place de la sage-femme dans la prise en charge de patientes atteintes de pathologies chroniques.

Plusieurs hypothèses en découlent :

1. Du fait de leur rôle fondamental en prévention, dépistage et éducation des femmes vis-à-vis de leur santé, et malgré une compétence qui dépasse le cadre de la physiologie, les sages-femmes se sentent impliquées face à ces patientes.

2. Les sages-femmes méconnaissent le concept récent de l'éducation thérapeutique. Elles ignorent qu'elles peuvent en être actrices. Elles pourraient même avoir les compétences de participer à des programmes d'éducation thérapeutique pour des femmes présentant des maladies chroniques pendant la grossesse (comme il en existe déjà pour le diabète).

3. Devant certaines pathologies chroniques comme l'épilepsie, les sages-femmes se sentent souvent démunies. Elles n'ont peut-être pas les moyens nécessaires et suffisants pour assurer ces prises en charge en terme de connaissances initiales et continues mais aussi du point de vue des aides qui leurs sont proposées (protocole, schéma thérapeutique...).

\subsection{Moyens de l'étude}

\subsubsection{Population étudiée}

Notre étude repose sur une enquête prospective, multicentrique par questionnaires anonymes distribués aux sages-femmes hospitalières, de PMI et libérales (ANNEXE X). 


\subsubsection{Distribution des questionnaires}

Nous souhaitions distribuer le questionnaire aux sages-femmes de Seine-Maritime et de l'Eure pour avoir un grand nombre de retours. Notre enquête se voulait également interroger les sages-femmes des maternités des trois niveaux.

Nous avons réalisé une étude préalable au mois de juillet 2011. Nous avons pris contact de manière téléphonique avec 14 cadres de maternité afin de présenter notre étude et obtenir leurs adresses mail.

Dans un deuxième temps, nous leur avons envoyé notre questionnaire par mail pour qu'ils l'étudient et nous donnent un accord préalable, une date de distribution et le nombre exact de sages-femmes de leurs services.

Pour le CHU, nous avons eu un entretien avec la sage-femme cadre supérieure.

Ainsi, notre distribution a pu débuter dès août 2011 dans 12 maternités de SeineMaritime et de 1'Eure.

\begin{tabular}{|l|c|}
\hline \multicolumn{1}{|c|}{ Maternités } & Nombre de questionnaires distribués \\
\hline \multicolumn{1}{|c|}{ Niveau 1 } & 8 \\
\hline Gisors & 20 \\
\hline l'Hôpital Privé de l'Estuaire (HPE) du Havre & 11 \\
\hline Bernay & 20 \\
\hline Lillebonne Niveau 2 & \\
\hline \multicolumn{1}{|c|}{} & 31 \\
\hline Evreux-Vernon Niveau 3 & 20 \\
\hline Dieppe & 13 \\
\hline Fécamp & 57 \\
\hline Le Belvédère & 36 \\
\hline La Clinique Mathilde & 32 \\
\hline Elbeuf & \\
\hline \multicolumn{1}{|c|}{} & 56 \\
\hline Jacques Monod & 65 \\
\hline CHU & \\
\hline
\end{tabular}

Pour toutes les maternités, nous avons fait le déplacement pour pouvoir solliciter l'équipe et nous présenter aux cadres sages-femmes des différents services.

Les cadres de Fécamp et Bernay ont géré eux-mêmes la reproduction, la distribution et la récupération des questionnaires. Ils nous les ont remis par voie postale en septembre 2011. Pour le CHU et les maternités de Jacques Monod et d'Elbeuf, nous avons distribué les questionnaires en septembre-octobre 2011. 
Les questionnaires ont été distribués de façon nominative. Il suffisait à chaque sagefemme de retirer la $1^{\text {ère }}$ page pour les rendre anonymes. Nous pensions, par cette démarche, intéresser un grand nombre de sages-femmes.

Les questionnaires nominatifs étaient disposés dans des enveloppes ou urnes mises à la disposition des professionnels dans les offices ou les bureaux.

Généralement, le retour des questionnaires s'est fait par voie postale, déplacement sur le terrain ou par l'intermédiaire de camarades de promotion.

Pour les sages-femmes de PMI et libérales d'Eure et de Seine-Maritime, les questionnaires ont été envoyés par voie postale, accompagnés d'un courrier explicatif et de nos coordonnées pour un éventuel retour. Nos 30 sages-femmes de PMI et libérales ont été contactées grâce aux terrains de stages proposés cette année et par l'aide d'une cadre du Belvédère.

La récupération des questionnaires a été arrêtée au 27 janvier 2012.

\subsubsection{Les difficultés rencontrées}

Notre enquête a été exhaustive en ce qui concerne la distribution mais sur 399 questionnaires distribués, seuls 160 questionnaires ont été récupérés. 9 d'entre eux ont été écartés car étaient mal remplis. Au total, 151 questionnaires ont été utilisés pour notre étude. Le taux de récupération est faible $\approx 38 \%$.

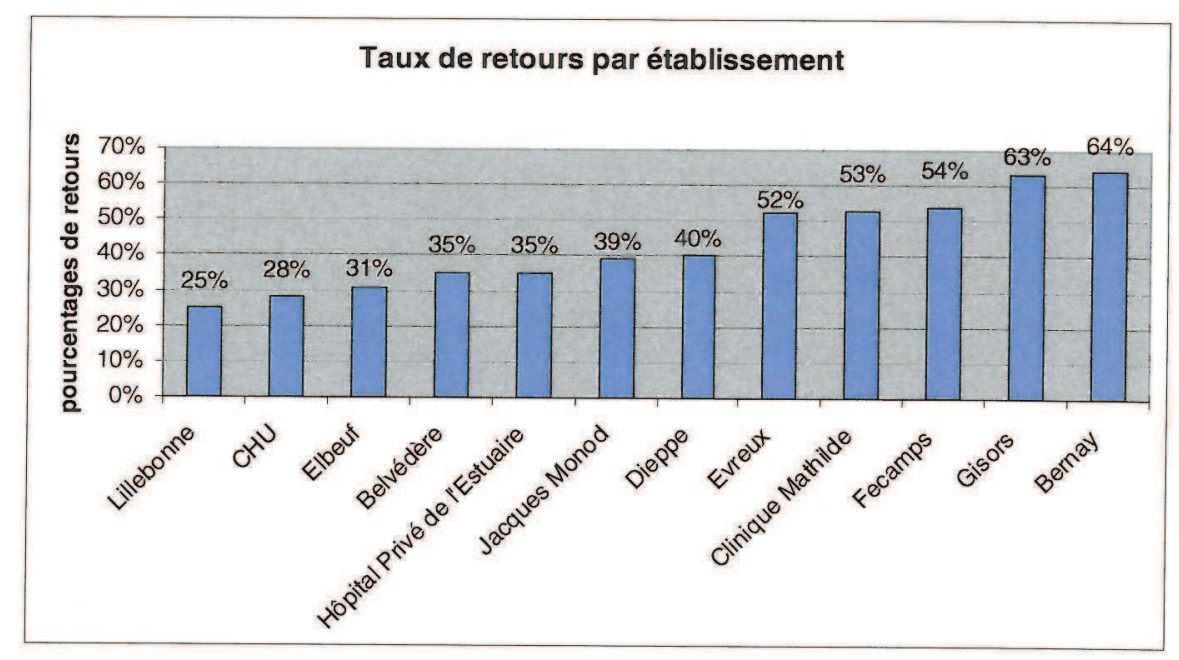

Le taux minimum de récupération est de $25 \%$, le taux maximum est de $64 \%$.

Pour les sages-femmes de PMI et libérales, 7 questionnaires sur 30 envoyés sont revenus soit $23 \%$ de récupération.

Nous savons que la population des sages-femmes est largement sollicitée pour répondre à de nombreux questionnaires d'étudiants. Notre questionnaire était par 
ailleurs long et compliqué du fait des questions ouvertes et nécessitant réflexion sur plusieurs sujets.

Les questions ouvertes, notamment celles justifiant les réponses « oui » ou « non », ont souvent suscité des non-réponses.

Les sages-femmes ont eu du mal à se restreindre à une seule réponse en ce qui concerne la pathologie chronique la plus rencontrée chez leurs patientes et leur justification quant à l'attention portée aux femmes malades chroniques (questions 7 et 10).

\subsection{Présentation des questionnaires}

Le questionnaire comporte 39 questions réparties en quatre parties.

La majorité des réponses est à choix multiples mais certaines questions nécessitent des réponses libres (âge, année du diplôme d'état, définitions, justifications...).

La première partie avait pour objet de dresser le profil de la population testée :

Le sexe : une variable qualitative à deux classes (féminin ou masculin)

$>$ L'âge : une variable quantitative continue, transformée en variable qualitative à huit classes : [20 à 25 ans[, [25 à 30 ans[, [30 à 35 ans[, [35 à 40 ans[, [40 à 45 ans[, [45 à 50 ans[, [50 à 55 ans[ et [55 à 60 ans[ .

$>$ L'année d'obtention du diplôme d'état : une variable quantitative continue, transformée en quatre classes pour évaluer l'ancienneté et l'expérience : [0 à 10 ans[, [10 à 20 ans[, [20 à 30 ans[ et [30 à 40 ans[ .

$>$ Le lieu d'exercice : une variable qualitative à quatre classes (hôpital public, clinique privée, cabinet libéral et PMI).

L'atteinte personmelle : une variable qualitative à deux classes (oui, non). Cette donnée permet de savoir si le fait d'être confronté (e) s à une ou plusieurs pathologies chroniques dans leur vie privée influence les connaissances et la prise en charge des sages-femmes dans ces situations.

La deuxième partie portait sur les pathologies chroniques en général. Nous voulions faire un état des lieux des connaissances des sages-femmes à ce sujet et leur ressenti vis-à-vis des patientes malades chroniques.

La définition de «pathologie chronique » : question ouverte dont les réponses ont été réunies en variables qualitatives à 8 classes (huit critères).

Les exemples de pathologies chroniques : question ouverte. 
La pathologie chronique la plus rencontrée au cours de la grossesse : question ouverte.

L'attention portée aux femmes enceintes atteintes de pathologies chroniques : une variable qualitative à deux classes (oui, non).

$>$ Le critère influençant ou non cette attention : variable qualitative à 9 classes.

Les facteurs influençant la non-observance d'un traitement: variable qualitative à 10 classes.

Les critères complexifiant la grossesse d'une patiente malade chronique pour la patiente elle-même et pour le professionnel qui la prend en charge : variable qualitative à 10 classes sous forme d'un VRAI/FAUX.

Dans la troisième partie, nous avons pu tester les connaissances des sages-femmes en matière d'éducation thérapeutique du patient et nous avons voulu savoir si ce concept les intéressait.

La connaissance de l'ETP : une variable qualitative à deux classes (oui, non).

$>$ Le lieu : question ouverte.

$>$ La définition d' «éducation thérapeutique » : question ouverte dont les réponses ont été réunies en variables qualitatives à 3 classes (bonnes réponses, mauvaises réponses et pas de réponses).

Les bonnes réponses comprennent : apprendre au patient à gérer son traitement, sa maladie ; apprendre à être autonome, acteur; accompagner, aider à vivre avec la maladie, le traitement; plusieurs intervenants; pour les pathologies chroniques; continu, prévention tertiaire.

Les mauvaises réponses comprennent: donner des informations, expliquer le traitement.

L'ETP dans leur pratique professionnelle : une variable qualitative à deux classes (oui, non). Les justifications : question ouverte.

$>$ La sage-femme dans des programmes d'ETP : une variable qualitative à deux classes (oui, non).

$>$ La sage-femme dans un programme d'ETP pendant la grossesse : une variable qualitative à deux classes (oui, non). Les justifications : question ouverte.

L'existence de formation en ETP : une variable qualitative à deux classes (oui, non). 
Les formations existantes : question ouverte dont les réponses ont été réunies en variables qualitatives à 4 classes.

Leur intérêt pour une formation d'ETP : une variable qualitative à deux classes (oui, non). Les justifications : question ouverte.

La quatrième partie de ce questionnaire permet d'illustrer la notion de pathologie chronique pendant la grossesse: la prise en charge des patientes épileptiques. Nous testons les sages-femmes sur leurs connaissances cette fois-ci en matière d'épilepsie et tentons de déterminer les outils supplémentaires qu'elles estiment nécessaires pour une meilleure prise en charge des ces femmes.

Le premier quizz sur l'épilepsie en général : variable qualitative à 4 classes selon le score sur 4 points obtenu.

\begin{tabular}{|c|c|c|c|}
\hline Questions & \multicolumn{3}{|c|}{ Points } \\
\hline $\begin{array}{l}\text { 1. Taux mondial des personnes } \\
\text { atteintes d'épilepsie : } 50 \text { millions }\end{array}$ & $\begin{array}{c}1 \\
\text { (bonne réponse sur la } \\
\text { flèche) }\end{array}$ & $\begin{array}{c}0,5 \\
\text { (valleur proche) }\end{array}$ & $\begin{array}{c}0 \\
\text { (valeur très } \\
\text { éloignée) }\end{array}$ \\
\hline $\begin{array}{l}\text { 2. Valeur seuil de crises épileptiques } \\
\text { pour parler d'épilepsie }: \geq 2 \text { crises }\end{array}$ & $\mathbb{1}$ & & \\
\hline $\begin{array}{l}\text { 3. Symptômes de l'épilepsie: } 7 \\
\text { symptômes }\end{array}$ & $\begin{array}{c}1 \\
\text { (7 bonnes réponses) }\end{array}$ & $\begin{array}{c}0,5 \\
\text { ( } \geq 4 \text { bonnes } \\
\text { réponses) }\end{array}$ & $\begin{array}{c}0 \\
(<4)\end{array}$ \\
\hline $\begin{array}{l}\text { 4. Conduites-à-tenir : } \\
\rightarrow \text { Alerter, appeler de l'aide (obstétricien, } \\
\text { anesthésiste, collègues) } \\
\rightarrow \text { Eviter les traumatismes (PLS, } \\
\text { protéger...) } \\
\rightarrow \text { Eviter l'asphyxie (canule de Guedel, } \\
\text { oxygène) } \\
\rightarrow \text { Vérifier les bruits du cœur fotaux } \\
\text { (enregistrement du rythme cardiaque, } \\
\text { sonicaid, éventuellement stéthoscope de } \\
\text { Pinard). } \\
\rightarrow \text { Préparer un anti convulsant et } \\
\text { l'administrer si besoin }\end{array}$ & $\begin{array}{c}1 \\
(\geq 4 \text { actions citées) }\end{array}$ & $\begin{array}{c}0,5 \\
(\geq 2 \text { actions } \\
\text { citées })\end{array}$ & $\begin{array}{c}0 \\
(<2 \text { actions } \\
\text { citées })\end{array}$ \\
\hline
\end{tabular}

Leur expérience face à une femme enceinte épileptique : une variable qualitative à deux classes (oui, non). 
Si oui, le ou les service(s) de cette prise en charge : variable qualitative à 14 classes.

Et les moyens auxquels elles ont eu recours : variable qualitative à 7 classes.

Si non, le ressenti qu'elles pourraient avoir face à cette situation : variable qualitative à 8 classes.

Le second quizz sur « épilepsie et grossesse » : variable qualitative à 4 classes selon le score sur 10 points obtenu.

\begin{tabular}{|c|c|c|c|c|c|}
\hline Questions & \multicolumn{5}{|c|}{ Points } \\
\hline $\begin{array}{l}\text { 1. Contraception : Les dispositifs intra-utérins sont } \\
\text { recommandés }\end{array}$ & \multicolumn{2}{|c|}{1} & \multicolumn{2}{|c|}{0} & \\
\hline $\begin{array}{l}\text { 2. Conception : Programmer sa grossesse. } \\
\text { Dose plus faible de son traitement avant la grossesse. }\end{array}$ & \multicolumn{2}{|c|}{$\begin{array}{c}1 \\
(2 \text { réponses }(\mathbf{r}))\end{array}$} & \multicolumn{2}{|c|}{$\begin{array}{c}0.5 \\
(1 \mathrm{r})\end{array}$} & 0 \\
\hline 3. L'échographie supplémentaire : 17-18 SA & \multicolumn{2}{|c|}{1} & \multicolumn{2}{|c|}{0} & \\
\hline $\begin{array}{l}\text { 4. Pourquoi ?: dépister les anomalies de fermeture du tube } \\
\text { neural }\end{array}$ & \multicolumn{2}{|c|}{1} & \multicolumn{2}{|c|}{0} & \\
\hline $\begin{array}{l}\text { 5. Les risques « épilepsie et grossesse » : } 5 \text { derniers risques } \\
\text { du tableau }\end{array}$ & \multicolumn{2}{|c|}{$\begin{array}{c}1 \\
(4 \text { ou } 5 \mathrm{r})\end{array}$} & \multicolumn{2}{|c|}{$\begin{array}{c}0.5 \\
(3 \mathrm{r})\end{array}$} & 0 \\
\hline 6. La molécule de la Dépakine $($ : valproate de sodium & \multicolumn{2}{|c|}{1} & \multicolumn{2}{|c|}{0} & \\
\hline 7. Continuer ce traitement : non & \multicolumn{2}{|c|}{1} & \multicolumn{2}{|c|}{0} & \\
\hline 8. Pourquoi ?: risques malformatifs & \multicolumn{2}{|c|}{1} & \multicolumn{2}{|c|}{0} & \\
\hline $\begin{array}{l}\text { 9. Informations sur la grossesse : } \\
\text { - doit bénéficier d'une supplémentation en folates } \\
\text { - l'angoisse n'est pas propice au déroulement de sa grossesse } \\
\text { - elle doit avoir un suivi régulier avec son neurologue } \\
\text { - son foetus a plus de risques de malformations }\end{array}$ & $\begin{array}{c}1 \\
(4 r)\end{array}$ & $\begin{array}{l}0.75 \\
(3 \mathrm{r})\end{array}$ & $\begin{array}{l}0.5 \\
(2 \mathrm{r})\end{array}$ & $\begin{array}{l}0.25 \\
(1 \mathrm{r})\end{array}$ & 0 \\
\hline 10. Risques du foetus : 4 risques attendus & $\begin{array}{c}1 \\
(4 \mathrm{r})\end{array}$ & $\begin{array}{l}0.75 \\
(3 \mathrm{r})\end{array}$ & $\begin{array}{l}0.5 \\
(2 \mathrm{r})\end{array}$ & $\begin{array}{l}0.25 \\
(1 \mathrm{r})\end{array}$ & 0 \\
\hline
\end{tabular}

$>$ Le besoin d'améliorer la prise en charge : une variable qualitative à deux classes (oui, non).

La présence de protocole de service : une variable qualitative à deux classes (oui, non).

$>$ L'aptitude à prendre en charge une femme épileptique à la sortie de l'école : une variable qualitative à deux classes (oui, non).

Les outils supplémentaires pour une prise en charge optimale : variables qualitatives à 8 classes. 


\subsection{Tests statistiques utilisés}

L'analyse statistique a été réalisée en utilisant le logiciel stata 7.0 (Stata Corporation, 4905 Lakeway Drive, College Station, Texas USA).

Dans le cadre de l'analyse uni-variée, les tests du Khi-2 et de Fisher ont été utilisés. Les différences observées ont été considérées statistiquement significatives pour p<0,05.

La représentation graphique des résultats a été réalisée par le logiciel Microsoft Office Excel 2003.

\section{Présentation des résultats}

$\underline{1^{\text {ère }} \text { partie : Profil de la population: }}$

\section{Question 1 :}

Sur 151 questionnaires remplis par les sages-femmes, nous avons 95\% de femmes $(n=143)$ et $5 \%$ d'hommes $(n=8)$.

\section{Question 2 : Quel est votre âge ?}

La moyenne des âges de notre population est de 34,43 ans. La médiane est à 32 ans. L'âge minimum est de 23 ans, l'âge maximum de 57 ans. Notre population est plus ciblée à la tranche d'âge 25-30 ans (44\% de notre population, n= 67 sages-femmes).

\section{Question 3 : En quelle année avez-vous obtenu votre diplôme d'état (DE) ?}

\section{Graphique $n^{\circ} 1$}

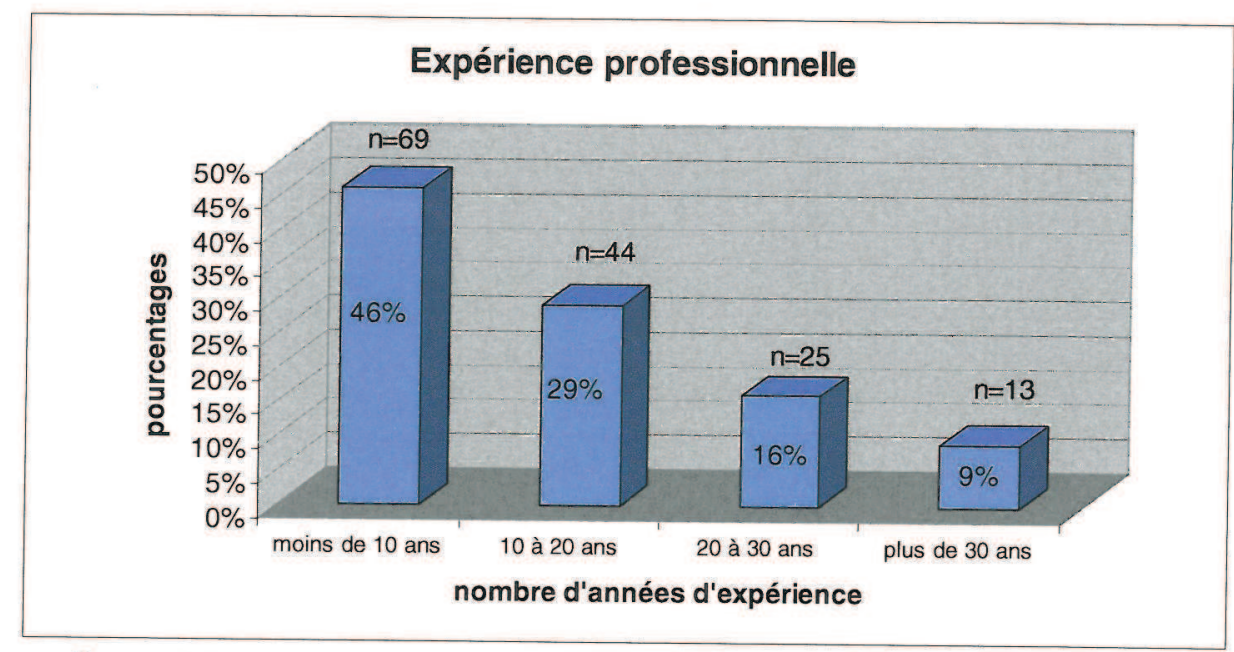

La médiane de notre échantillon est de 8 ans d'expérience professionnelle. La catégorie de professionnels la plus représentée est celle des moins de 10 ans d'ancienneté. 


\section{Question 4 : Quel est votre lieu d'exercice?}

\section{Graphique ${ }^{\circ} 2$}

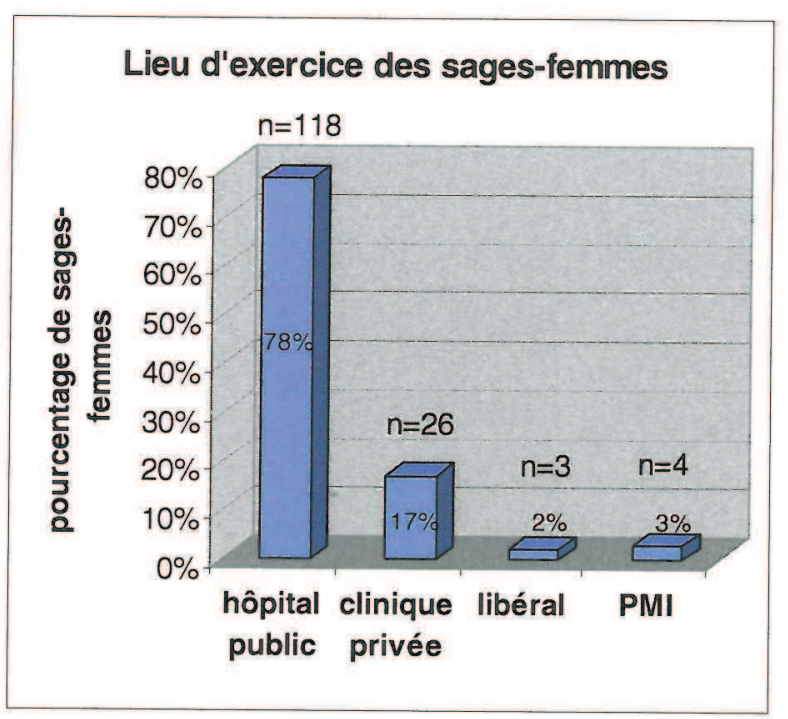

Notre étude a majoritairement été réalisée en milieu public hospitalier (78\%).

Nous avons recensé 24 sages-femmes de niveau 1 (17\%), 80 travaillant en niveau 2 (55\%) et 40 en niveau 3 (28\%).

\section{Question 5 : Etes-vous déjà confronté (e), à titre personnel, à une pathologie chronique?}

Plus d'un quart des sages-femmes de notre étude $(n=44)$ déclare être confronté, à titre personnel, à une pathologie chronique.

\section{$\underline{2^{\text {ème }}}$ partie : A propos des pathologies chroniques ...}

\section{Question 6 : Donnez votre propre définition de « pathologie chronique » :} Après avoir répertorié les principales idées données par les sages-femmes à cette question, nous en avons déduit huit critères. Certaines sages-femmes ont mentionné plusieurs critères dans leur définition.

Tableau $\mathrm{n}^{\circ} 1$

\begin{tabular}{|l|c|}
\hline \multicolumn{1}{|c|}{ Critères } & Fréquence de citation \\
\hline «longue durée, à vie » & 58 fois \\
\hline « traitement et prise en charge à vie » & 44 fois \\
\hline «long et incurable » & 34 fois \\
\hline «évolutive » & 21 fois \\
\hline « pré-existente à la grossesse » & 18 fois \\
\hline « répercussions sur la vie », «plus ou moins & 7 fois \\
\hline invalidante » & 4 fois \\
\hline « atteinte d'un organe ou d'un système » & 1 fois \\
\hline « maladie congénitale et/ou acquise » & \\
\hline
\end{tabular}


La notion de durée dans le temps de la maladie revient fréquemment dans la définition donnée par les professionnels.

Quatre sages-femmes (3\% de notre population) ont donné une définition précise de la «pathologie chronique » en utilisant trois des critères ci-dessus.

$21 \%$ d'entre elles $(n=32)$ l'ont défini en utilisant deux critères et les $76 \%(n=115)$ restant on donné une définition avec un critère : la durée.

Nous avons voulu savoir si le fait d'être confronté, à titre personnel, à une pathologie chronique influençait la réponse à cette question.

Graphique $n^{\circ} 3$

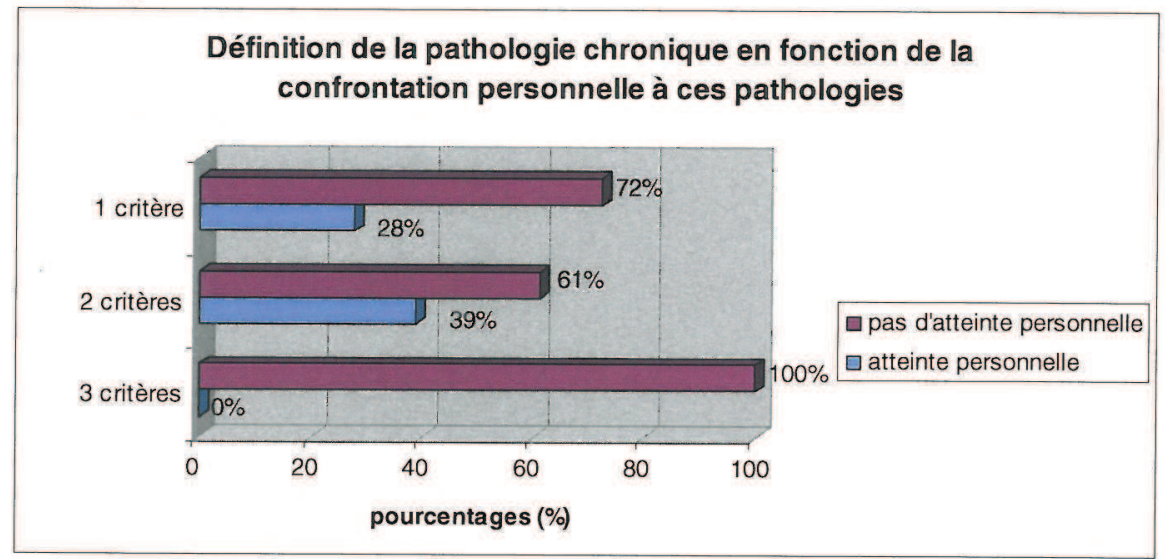

Le test de Fisher réalisé montre que les meilleures définitions sont données par les sages-femmes non confrontées à titre personnel aux pathologies chroniques. Ce test est non significatif $(\mathrm{p}=0.24)$.

\section{Question 7 : Donnez 3 exemples de pathologies chroniques}

\section{Graphique $\mathbf{n}^{\circ} 4$}

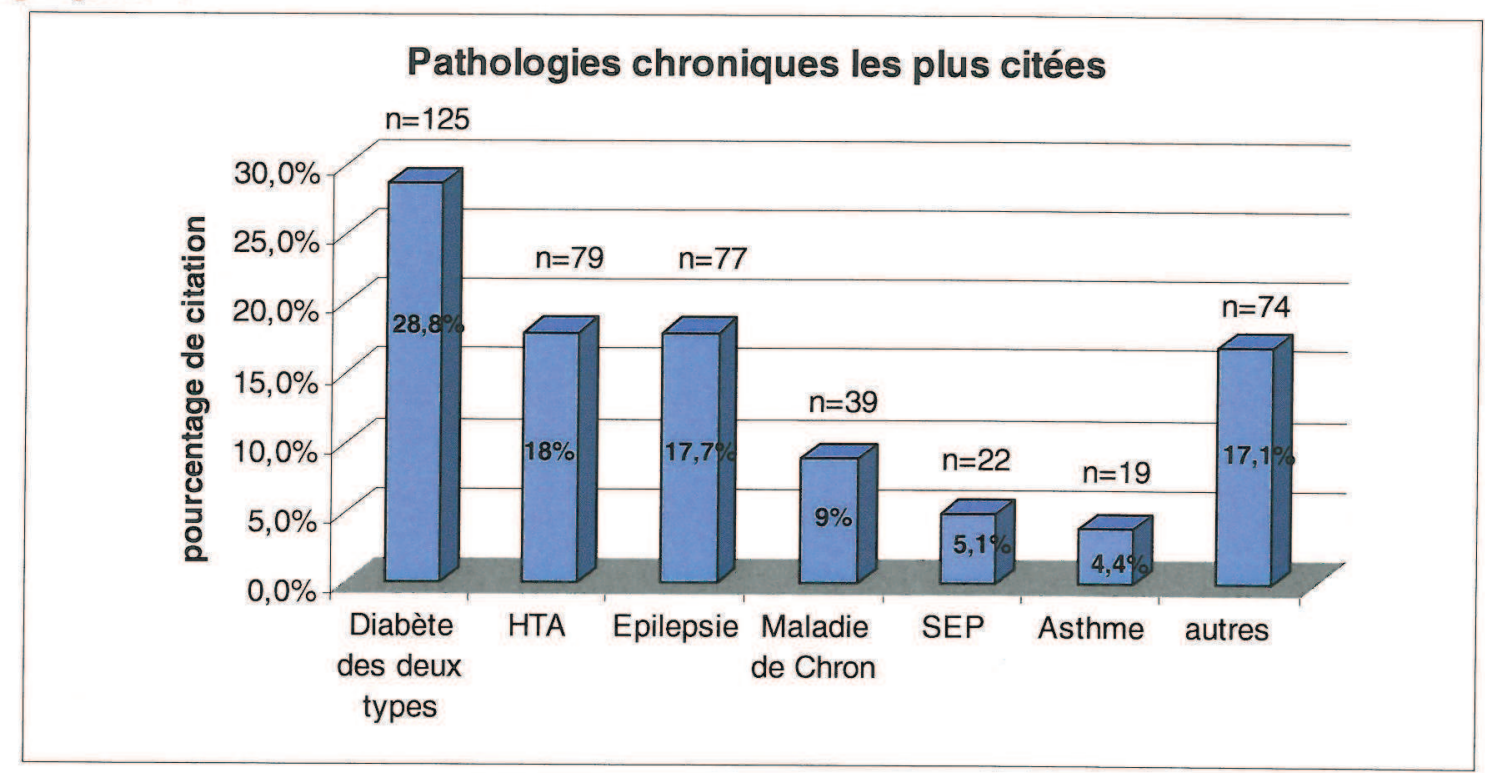


Le trio des pathologies chroniques les plus citées est : diabète insulinodépendant et non insulinodépendant, l'hypertension artérielle chronique suivie de l'épilepsie. Nous pensons que cette dernière est biaisée par notre questionnaire.

\section{Graphique ${ }^{\circ} 5$}

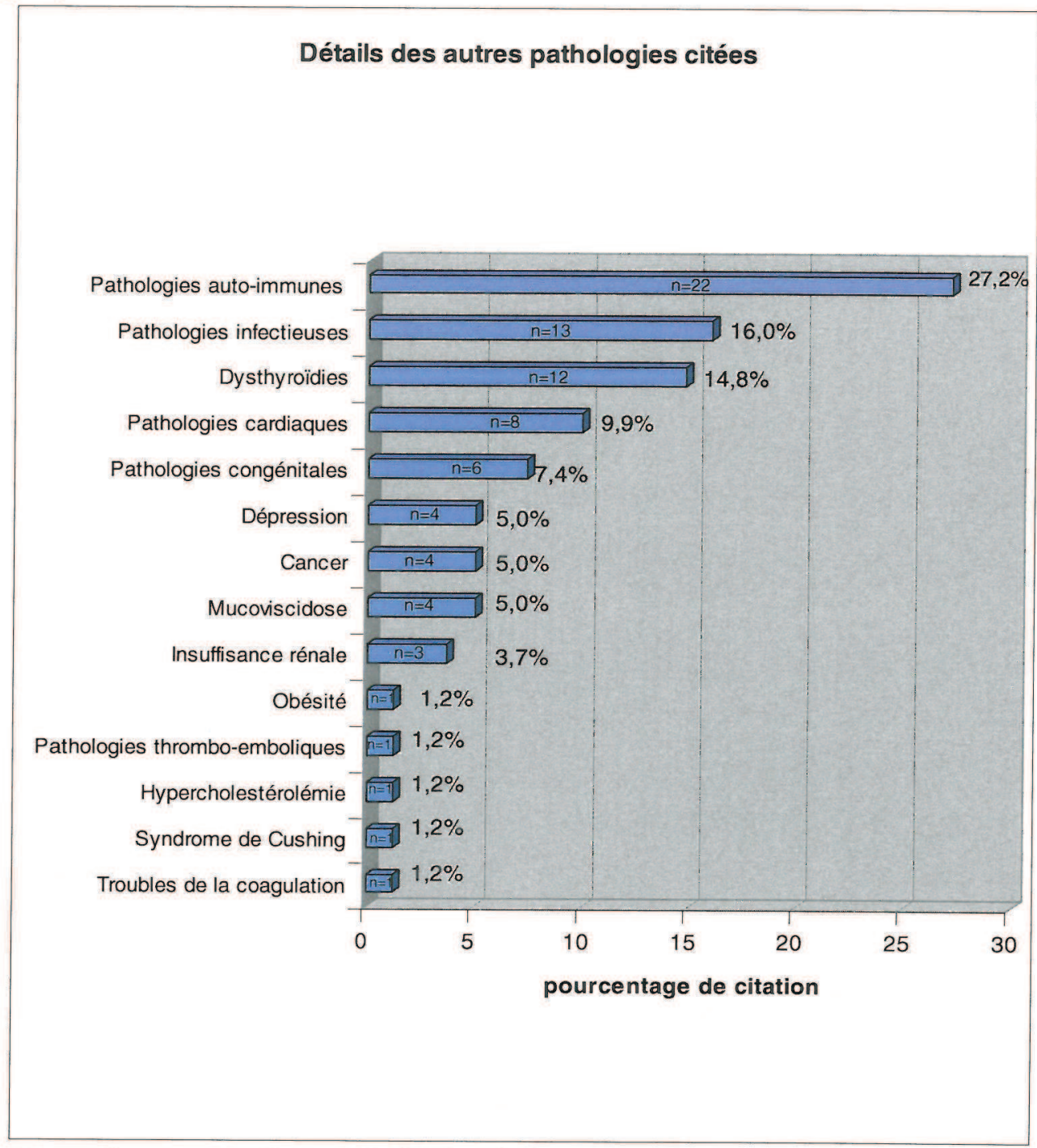

Dans le groupe des pathologies infectieuses, nous retrouvons les hépatites et l'infection à VIH.

Le taux de non réponse à cette question a été de $4 \%$ (n=6 sages-femmes). 
Question 8 : Quelle pathologie chronique rencontrez-vous le plus souvent chez vos patientes ?

Graphique $n^{\circ} 6$

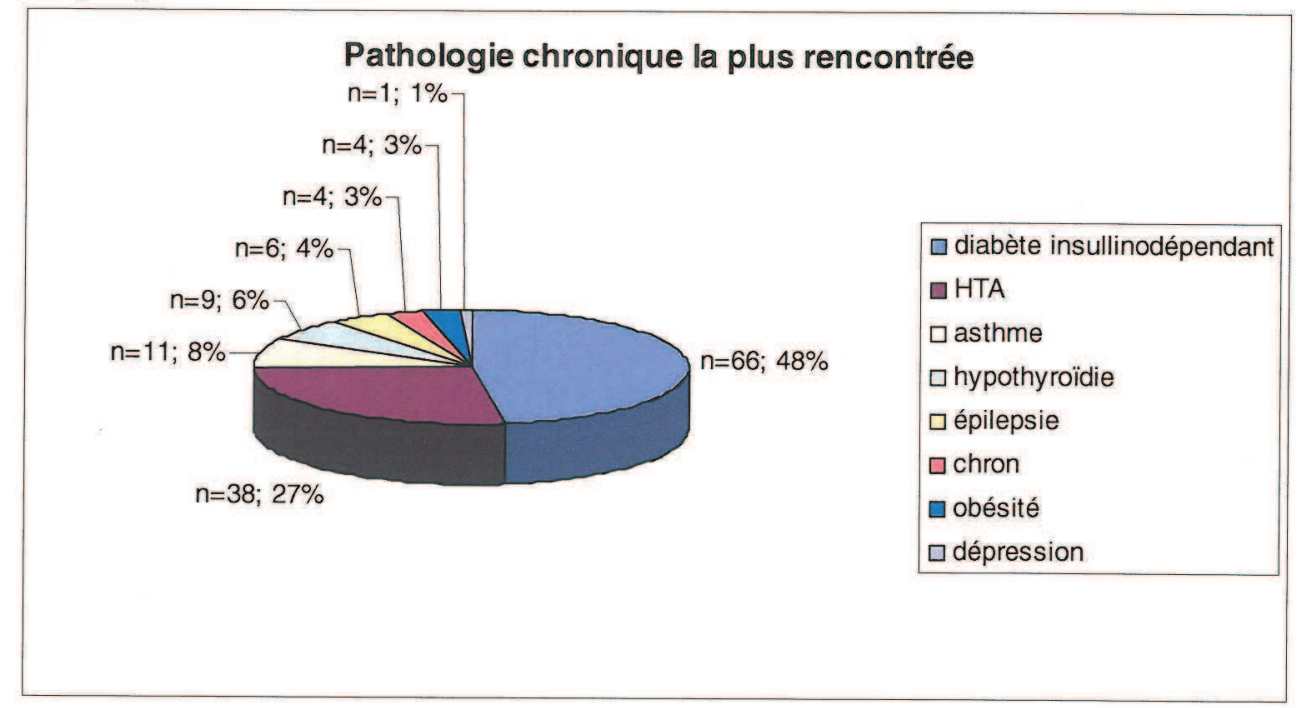

$8 \%$ des sages-femmes ont mal répondu à cette question: une personne n'a pas répondu et les autres n'ont pas respecté la consigne (trois pathologies ont été citées au lieu d'une seule).

La pathologie chronique la plus largement citée par notre population est le diabète insulinodépendant (48\% des réponses). Il est suivi de 1'hypertension artérielle chronique mentionnée dans $27 \%$ des réponses.

Question 9: Portez-vous une attention plus particulière aux femmes enceintes atteintes de pathologies chroniques?

Une personne n'a pas répondu à cette question.

$94 \%$ d'entre elles $(\mathrm{n}=141)$ ont répondu accorder une attention plus particulière aux femmes enceintes atteintes de pathologies chroniques. Seulement $6 \%$ d'entre elles ont répondu « non ».

Une fois encore, nous avons analysé si la notion de confrontation à la maladie à titre personnel pouvait influencer l'attention portée à ces femmes. 


\section{ERRATUM}

Je vous prie d'excuser mon erreur sur le graphique $\mathrm{n}^{\circ} 7$ (page 32) :

Ce graphique :

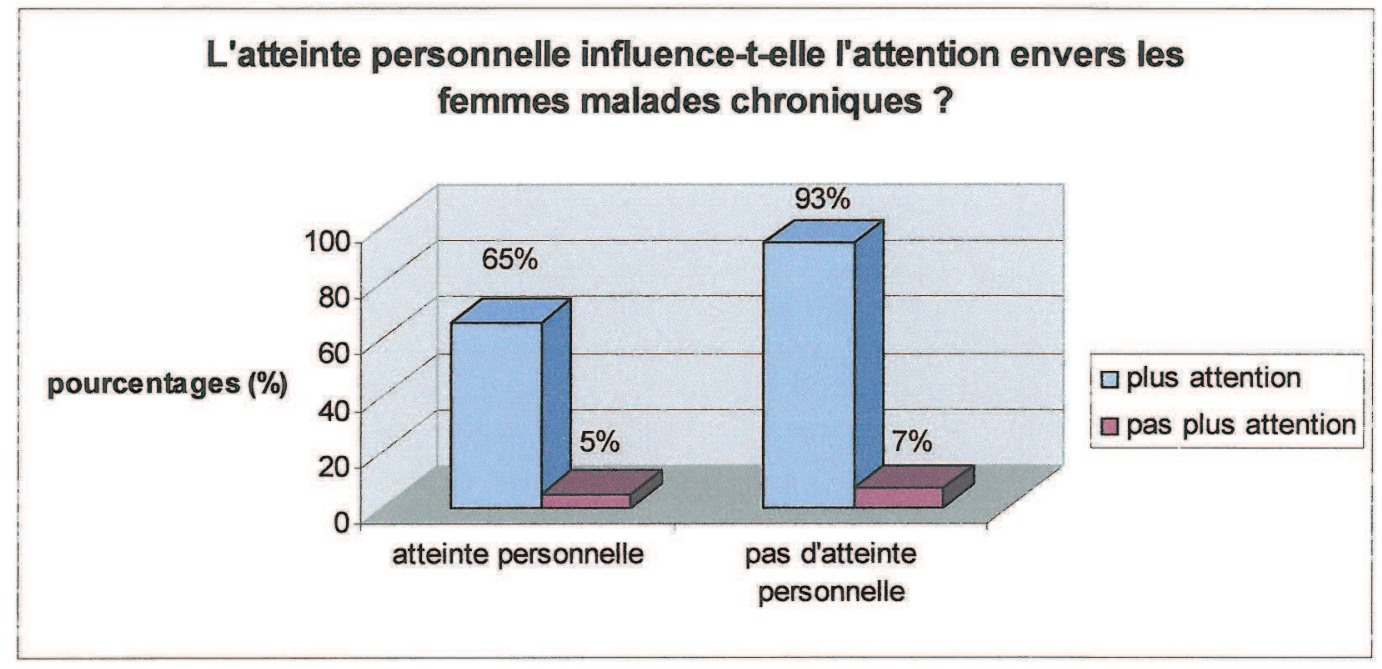

doit être remplacé par celui-ci :

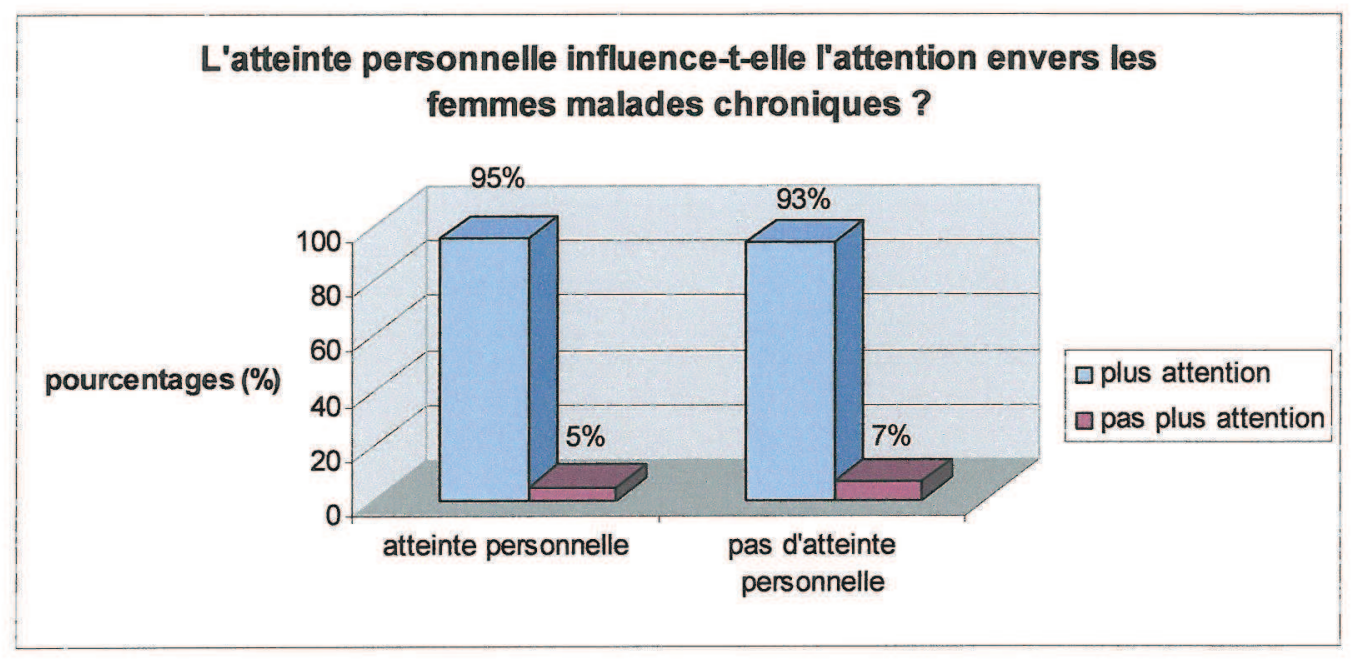

L'atteinte personnelle n'influence pas l'attention portée à une patiente malade chronique (test de Fisher $(\mathrm{p}=1)$ ).

Par contre, on ne peut plus dire que le pourcentage des sages-femmes portant plus d'attention est plus élevé dans le groupe « pas d'atteinte personnelle ». 


\section{Graphique ${ }^{\circ} 7$}

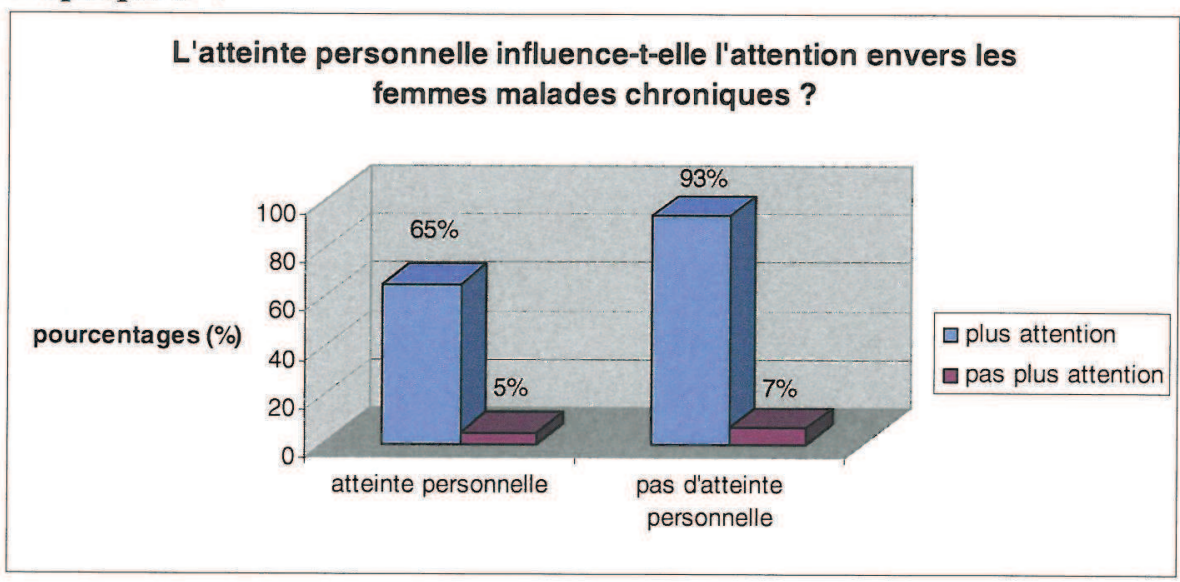

Par le test de Fisher, nous faisons le constat que l'atteinte personnelle n'influence pas l'attention portée à une patiente malade chronique. Le pourcentage des sagesfemmes portant plus d'attention est d'ailleurs plus élevé dans le groupe «pas d'atteinte personnelle ». Ce test est non significatif $(p=1)$.

\section{Question 10 : Pourquoi ?}

Pour cette question, nous avions souhaité une seule réponse afin de connaître la plus pertinente du praticien interrogé. 31 sages-femmes ( $21 \%$ de notre population) n'ont pas respecté cette consigne.

\section{Graphique $n^{\circ} 8$}

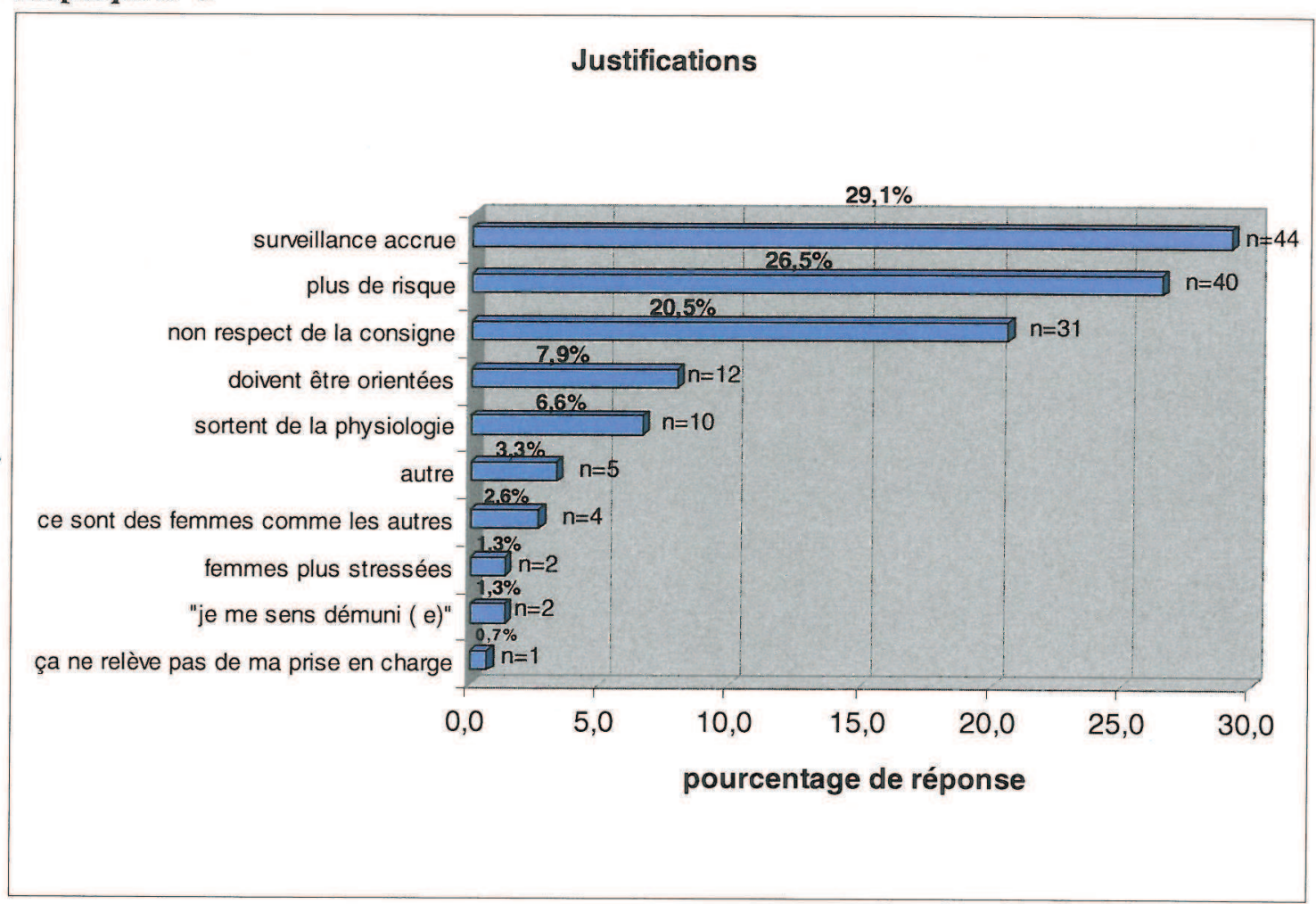


$55,6 \%$ des sages-femmes $(n=84)$ de notre échantillon ont souligné le caractère dangereux de ces grossesses (« surveillance accrue », «plus de risques »).

$14,5 \%$ d'entre elles ont rappelé le caractère pathologique de la grossesse d'une patiente malade chronique («les femmes sortent de la physiologie», «elles doivent être orientées »).

Parmi celles n'accordant pas d'attention particulière, une sage-femme déclare que les femmes enceintes malades chroniques ne relèvent pas de sa prise en charge. Au contraire, quatre sages-femmes considèrent ces femmes comme les autres.

Dans les réponses « autre » (3.3\% des réponses), il est précisé que « les femmes sont déjà prises en charge en dehors de la grossesse et ne nécessitent pas plus d'attention ». Une autre sage-femme déclare que l'attention dépend de la pathologie de la patiente.

\section{Question 11: A quoi est liée la non-observance ?}

Pour cette question, plusieurs réponses étaient possibles. Ainsi, nous avons relevé les facteurs influençant la non-observance en fonction de la fréquence à laquelle ils ont été cités :

Tableau $n^{\circ} 2$

\begin{tabular}{|l|c|}
\hline \multicolumn{1}{|c|}{ Facteurs } & Fréquence \\
\hline Le déni de la maladie & 92 fois \\
\hline Le niveau socio-économique & 88 fois \\
\hline Les effets secondaires du traitement & 66 fois \\
\hline Le manque d'informations & 49 fois \\
\hline La complexité du traitement & 45 fois \\
\hline Le fait que la pathologie soit dépistée depuis longtemps & 43 fois \\
\hline L'âge de la patiente & 39 fois \\
\hline Le fait que la pathologie soit dépistée depuis peu de temps & 28 fois \\
\hline La polythérapie & 26 fois \\
\hline Autre & 3 fois \\
\hline
\end{tabular}

Dans les réponses « autre », il est précisé que le « manque de compréhension de la gravité de la pathologie » et la « grossesse » peuvent influencer la non-observance des traitements.

Questions 12 et 13: Par quoi la grossesse d'une patiente atteinte d'une pathologie chronique est compliquée pour elle? et pour vous, en tant que professionnel qui la prenez en charge ?

Pour ces deux questions, nous avons estimé qu'un facteur complique la grossesse lorsque plus de $50 \%$ des sages-femmes répondent « VRAI ». 
Tableau $n^{\circ} 3$

\begin{tabular}{|c|c|c|}
\hline Facteurs & $\begin{array}{c}\text { VRAI } \\
\text { Pour la patiente } \\
\text { (réponses données par les } \\
\text { sages-femmes) }\end{array}$ & $\begin{array}{c}\text { VRAI } \\
\text { Pour la sage-femme }\end{array}$ \\
\hline \multicolumn{3}{|c|}{ Facteurs compliquant la grossesse } \\
\hline Les examens paracliniques supplémentaires & $56 \%$ & $54 \%$ \\
\hline La multiplicité des professionnels intervenants & $71 \%$ & $52 \%$ \\
\hline Les risques encourus par le foetus & $89 \%$ & $83 \%$ \\
\hline Les risques encourus par la femme & $74 \%$ & $83 \%$ \\
\hline $\begin{array}{l}\text { Les interactions médicamenteuses possibles avec } \\
\text { les traitements }\end{array}$ & $54 \%$ & $72 \%$ \\
\hline $\begin{array}{l}\text { Les possibles contre-indications des traitements } \\
\text { avec la grossesse }\end{array}$ & $87 \%$ & $75 \%$ \\
\hline $\begin{array}{l}\text { Les possibles contre-indications des traitements } \\
\text { avec l'allaitement }\end{array}$ & $77 \%$ & $71 \%$ \\
\hline $\begin{array}{l}\text { Le besoin d'équilibration de la pathologie pour et } \\
\text { pendant la grossesse }\end{array}$ & $82 \%$ & $84 \%$ \\
\hline \multicolumn{3}{|c|}{ Facteurs compliquant peu ou pas la grossesse } \\
\hline les problèmes psycho-sociaux liés à la pathologie & $42 \%$ & $36 \%$ \\
\hline
\end{tabular}

Nous observons que les interactions médicamenteuses possibles avec les traitements compliquent davantage la grossesse pour le praticien. A l'inverse, les sages-femmes pensent que la multiplicité des professionnels intervenants est une complication perçue plus par la femme enceinte.

Selon notre population, les problèmes psycho-sociaux liés à la pathologie ne compliquent pas ou peu la grossesse.

$\underline{3^{\text {ème }} \text { partie : A propos de l'éducation thérapeutique ... }}$

Question 14: Avez-vous déjà entendu parler de l' «éducation thérapeutique du patient $»$ ?

89\% des sages-femmes ont connaissance de l'éducation thérapeutique du patient. 
Si oui, à quelle(s) occasion(s) ?

Graphique ${ }^{\circ} 9$

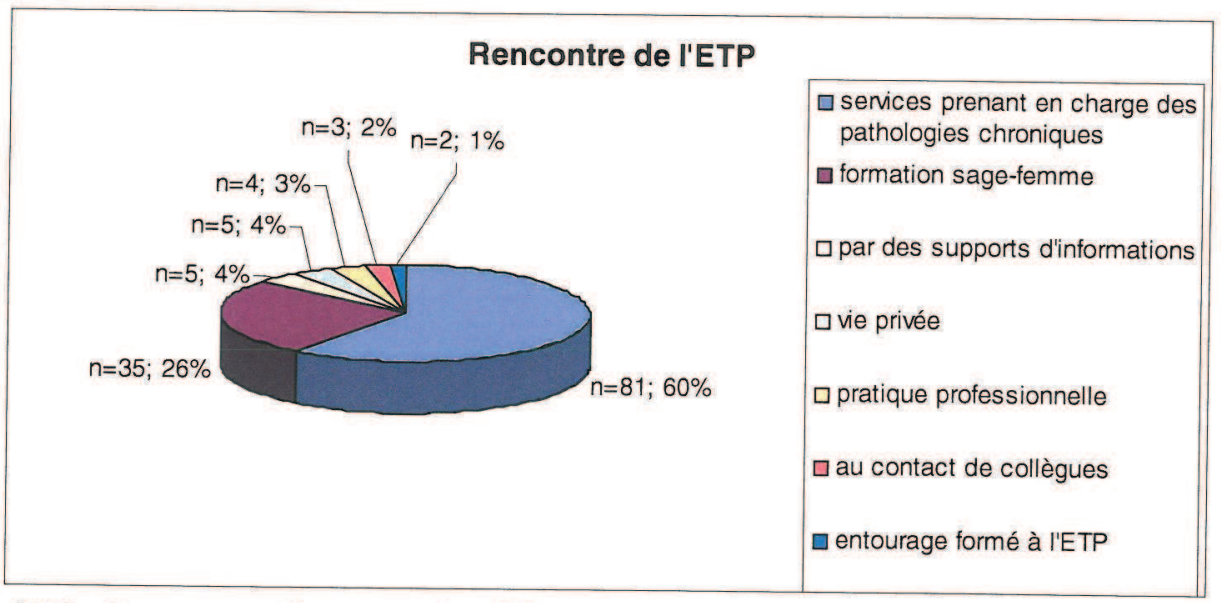

$60 \%$ des sages-femmes $(\mathrm{n}=81)$ ont entendu parler d'ETP au sein d'un service prenant en charge des pathologies chroniques. Pour 79 d'entre elles, il s'agit d'une unité de diabétologie.

La formation sage-femme (études et terrains de stage) a permis à 35 personnes de rencontrer l'éducation thérapeutique (26\%).

Seulement 3\% d'entre elles $(n=4)$ déclarent avoir entendu parler d'ETP au cours de leur pratique professionnelle.

Les supports d'information mentionnés par notre population sont la télévision, les colloques, les articles et notre questionnaire.

Question 15: Donnez votre définition d' «éducation thérapeutique du patient » :

Graphique $n^{\circ} 10$

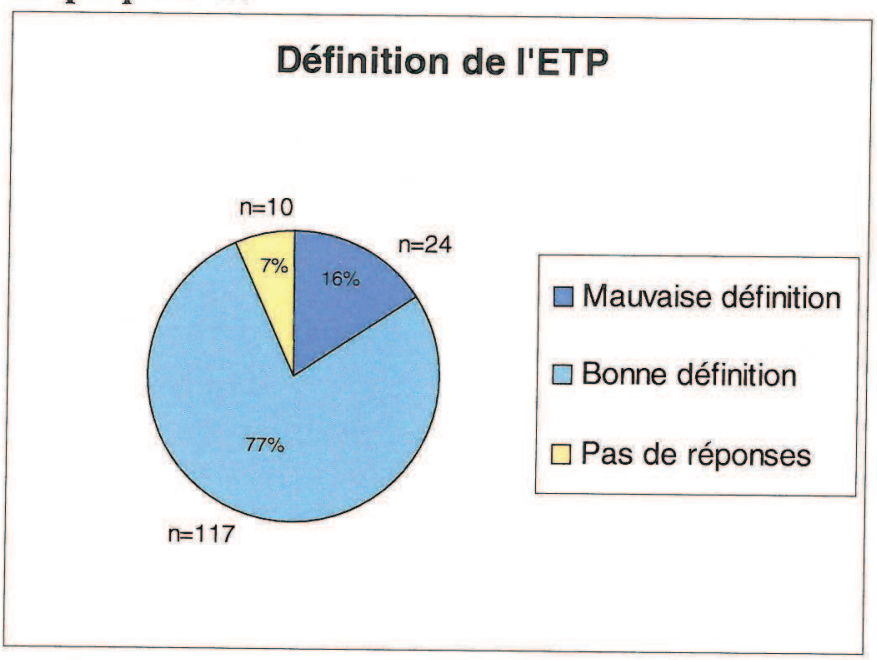

Parmi les mauvaises réponses étaient citées « informations » ou « explications de la pathologie ».

Les sages-femmes ayant donné la bonne réponse connaissaient au moins un des items de la définition. (cf méthodologie). 


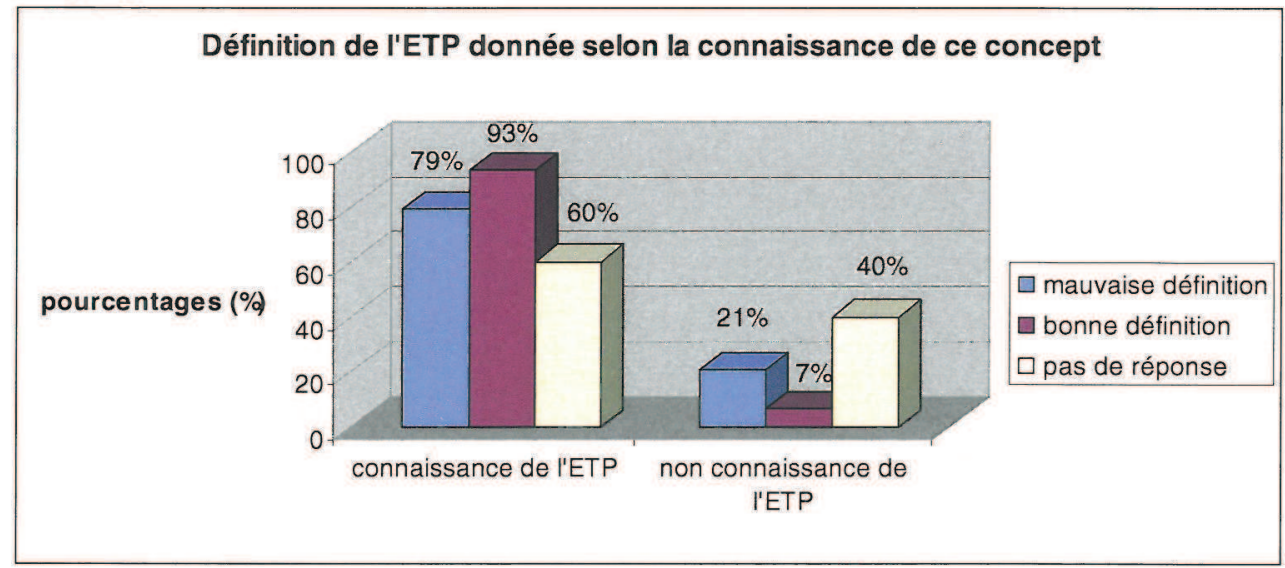

La connaissance de l'existence de l'ETP influence de manière significative (Test du chi2 : $\mathrm{p}=0.002$ ) la définition donnée de l'ETP.

Les sages-femmes qui ont déjà entendu parler de l'ETP donnent plus de bonnes réponses que de mauvaises réponses.

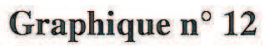

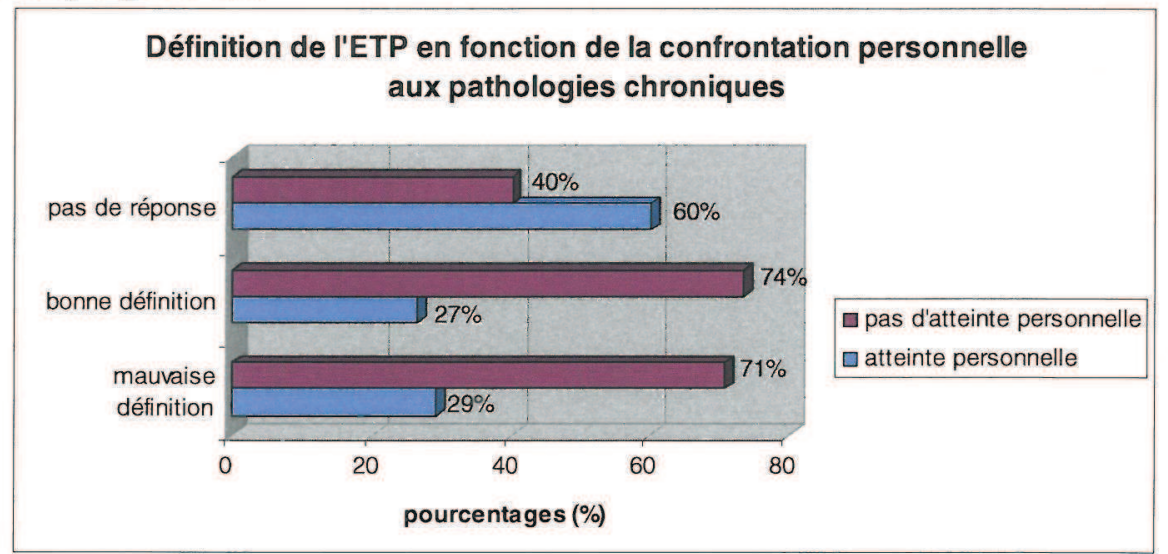

Le test de Fisher montre, avec une forte tendance $(\mathrm{p}=0.06)$, que la confrontation personnelle aux pathologies chroniques n'influence pas la définition donnée de l'ETP. Les taux de mauvaises ou bonnes réponses sont presque identiques quelque soit l'atteinte personnelle ou non.

Nous pouvons même remarquer que le taux de non réponse à cette question est plus important (60\%) en cas d'atteinte personnelle.

\section{Question 16 : Dans votre profession, pensez-vous éduquer les patientes ?}

Le taux de non réponse à cette question est de $2 \%$.

Plus de $80 \%$ des sages-femmes $(\mathrm{n}=121)$ pensent faire de l'éducation thérapeutique dans leur profession. 
Si oui, dans quelle(s) situations ?

Graphique ${ }^{\circ} 13$

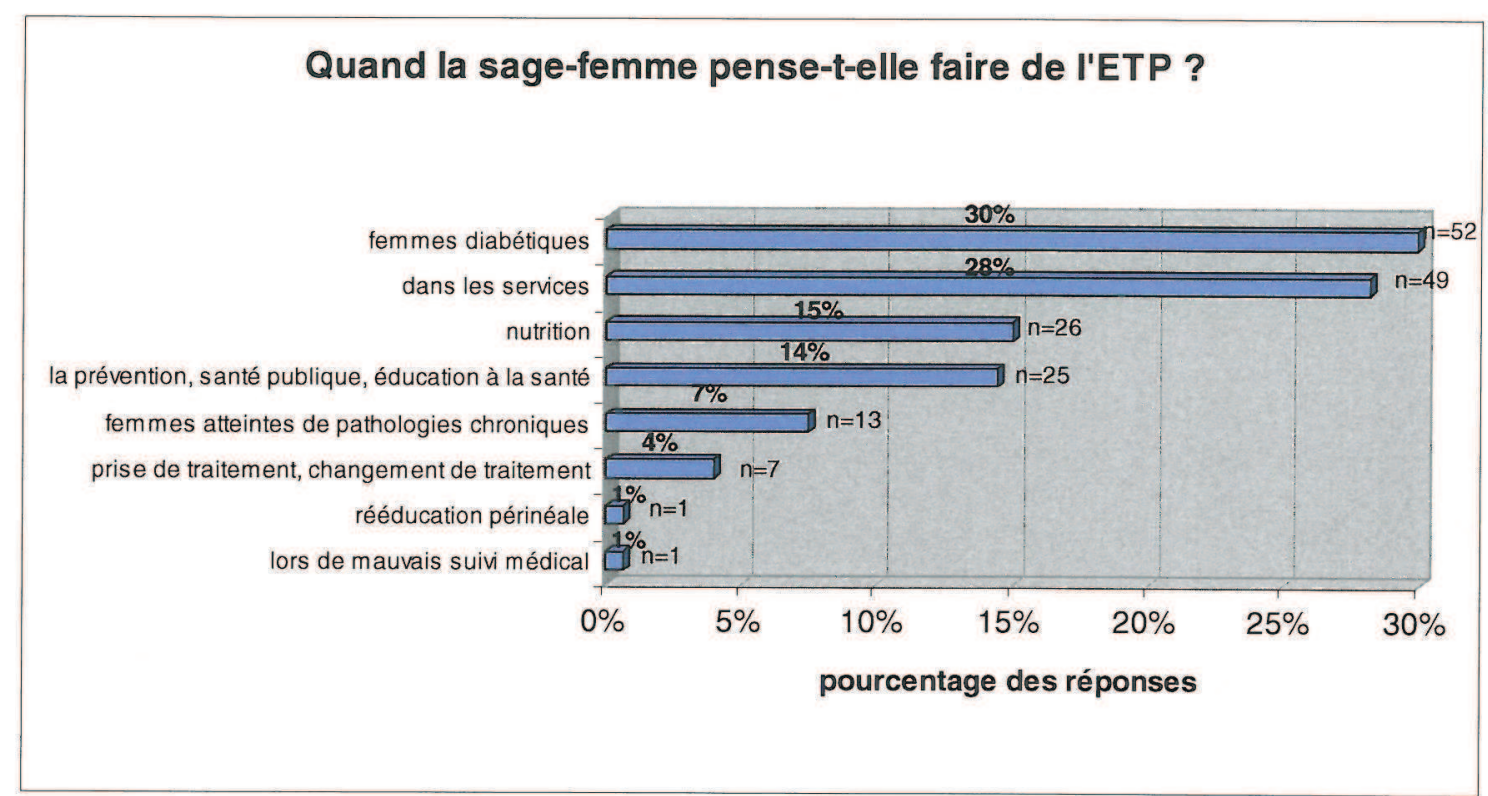

L'éducation thérapeutique des femmes diabétiques a été fortement mentionnée par nos sages-femmes.

La nutrition avec l'éducation alimentaire est mentionnée en troisième place.

Les services mentionnés sont les consultations prénatales et/ou d'urgences, le service de suites-de-couche, l'unité de grossesses pathologiques et la salle de naissance (gérer le travail, les efforts expulsifs).

En ce qui concerne la prévention, nous retrouvons la contraception, les addictions, l'hygiène de vie. 
Si non, pourquoi ?

Graphique n ${ }^{\circ} 14$

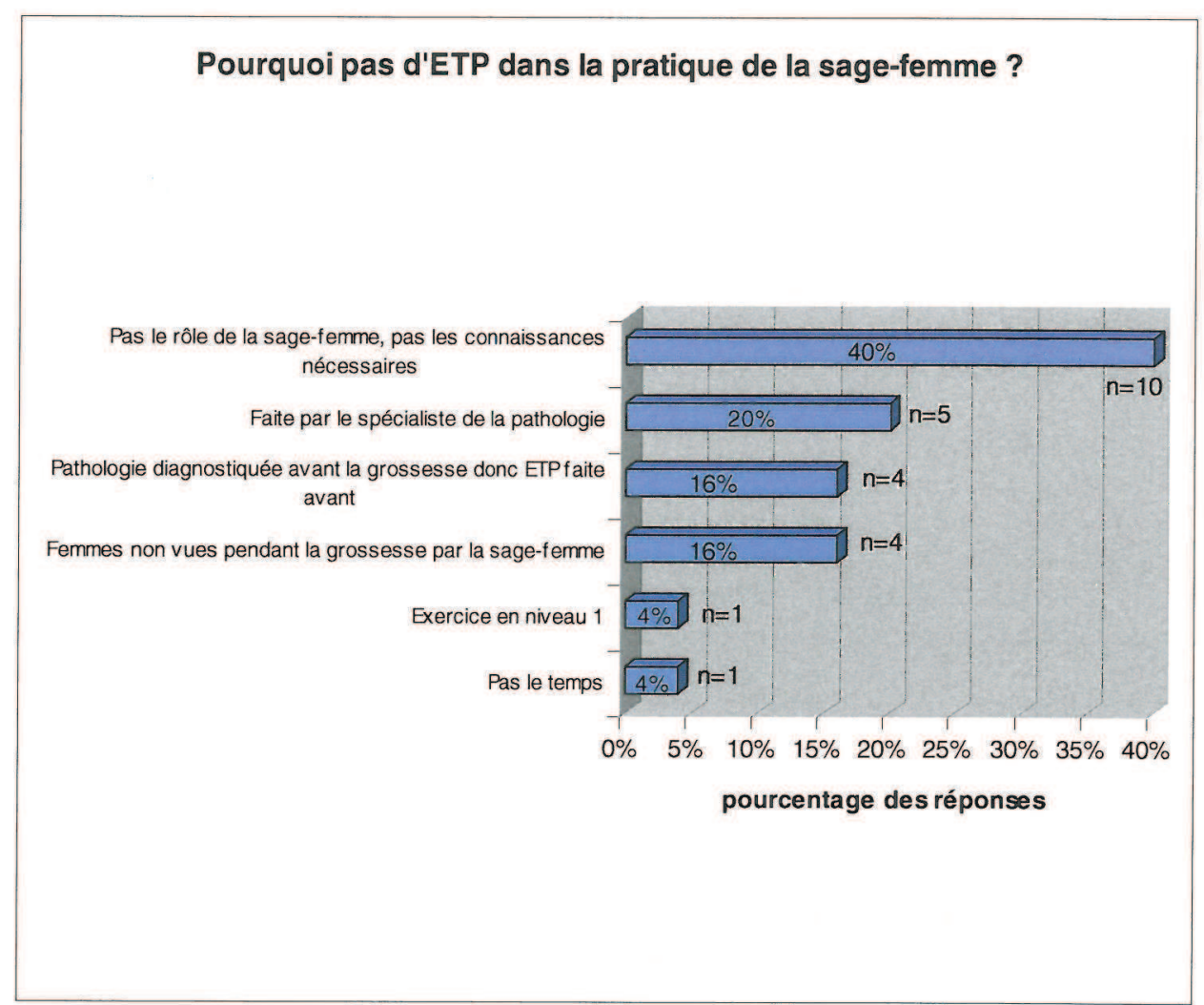

$40 \%$ des sages-femmes déclarant ne pas pratiquer d'ETP dans leur profession précisent qu'elles n'ont pas les connaissances nécessaires et qu'il ne s'agit pas de leur rôle.

Le rôle prédominant du médecin est souligné pour 36\% d'entre elles (« ETP faite par le spécialiste de la pathologie » et «Femmes non vues pendant la grossesse par la sagefemme $\gg$ ).

Question 17: Pensez-vous que la sage-femme peut s'inscrire dans des programmes d'éducation thérapeutique du patient ?

Les sages-femmes ont répondu « oui » pour plus de $90 \%$ d'entre elles $(n=136) .8 \%$ ont répondu défavorablement. Le taux de non réponse est faible de $2 \%$.

Pensez-vous que la sage-femme pourrait avoir une place dans un programme d'éducation thérapeutique destiné aux femmes atteintes de pathologies chroniques pendant la grossesse ?

Plus des trois quarts de notre population $(n=123)$ est favorable à ce que la sagefemme ait une place dans un programme d'ETP au cours de la grossesse. Le taux de non réponse est de $2,6 \%$. 


\section{Pourquoi oui ?}

\section{Graphique $n^{\circ} 15$}

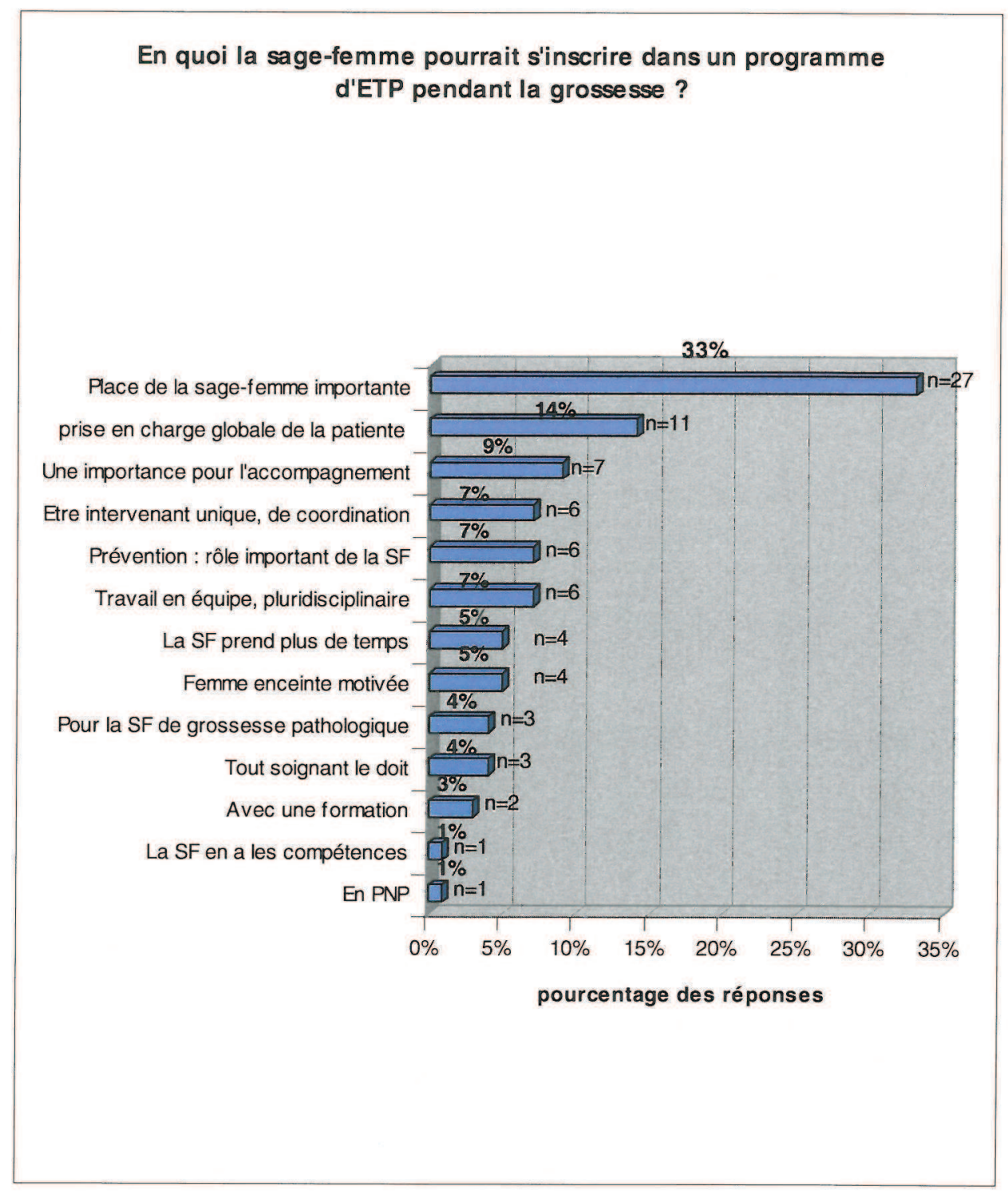

Les personnes favorables à cette proposition justifient dans un tiers des réponses que la place de la sage-femme est importante au cours de la grossesse. La globalité de la prise en charge est évoquée dans $14 \%$ des cas.

Nous remarquons que $5 \%$ des sages-femmes $(n=4)$ pensent prendre plus de temps avec leurs patientes.

\section{Pourquoi non ?}

Sur les 24 sages-femmes non favorables à cette idée, dix n'ont donné aucune justification (42\% des réponses).

$38 \%$ d'entre elles $(n=9)$ estiment que l'ETP sort de la physiologie.

Dans $8 \%$ des réponses ( $\mathrm{n}=2$ sages-femmes), elles pensent ne pas avoir assez de temps. $8 \%$ également déclarent que les patientes sont déjà prises en charge avant la grossesse. 
Enfin, une sage-femme précise que l'ETP ne serait utile que dans le service de grossesses pathologiques.

\section{Question 18: Pensez-vous qu'il existe une ou des formations en matière} d'éducation thérapeutique?

Un peu plus de la moitié des sages-femmes $(\mathrm{n}=80)$ a répondu favorablement à cette question. Un tiers d'entre elles a répondu qu'il n'existe pas de formation $(n=51) .13 \%$ n'ont donné aucune réponse $(\mathrm{n}=20)$.

\section{Question 19: Quel(s) types de formations?}

Près des deux tiers de notre population $(n=100)$ ne connaissent pas les formations d'ETP. 7\% des sages-femmes $(\mathrm{n}=10)$ pensent à un diplôme universitaire (DU) d'ETP et $7 \%$ mentionne le DU de pathologies maternelles et grossesse.

31 sages-femmes (21\%) ont donné de mauvaises réponses (stages, tables rondes, autres DU...).

\section{$>$ Question 20 : Seriez- vous intéressé (e) par une de ces formations ?}

$63 \%$ des sages-femmes interrogées $(\mathrm{n}=95)$ ont montré un intérêt pour une formation en ETP. Le taux de non réponse à cette question est de $4 \%$.

\section{Question 21: Pourquoi ?}

Tableau $n^{\circ} 4$

\begin{tabular}{|l|c|}
\hline \multicolumn{1}{|c|}{ Réponses } & Nombre de sages-femmes \\
\hline Encadrer mieux, être plus à l'aise & 33 \\
\hline Enrichissement théorique et pratique & 16 \\
\hline Dans le cadre de la formation continue & 12 \\
\hline Pour une meilleure compréhension des patientes, adapter son discours & 9 \\
\hline ETP pluridisciplinaire, travailler en équipe & 4 \\
\hline La sage-femme est en première ligne & 3 \\
\hline L'ETP pourrait avoir un intérêt en PMI & 1 \\
\hline Les pathologies chroniques sont en augmentation & 1 \\
\hline Formation déjà faite & 1 \\
\hline
\end{tabular}

Parmi les 95 sages-femmes intéressées par une formation en ETP, près d'un tiers pense que celle-ci pourrait améliorer leur prise en charge de femmes enceintes malades chroniques. 15 sages-femmes (16\% d'entre elles) n'ont pas explicité leur intérêt pour une formation d'ETP.

\section{Pourquoi non?}

50 sages-femmes (33\% de notre population) ne portent pas d'intérêt pour une formation d'ETP. 
Parmi elles, $23 \%$ sont simplement non intéressées $(n=10)$.

$30 \%$ ne le sont pas pour le moment $(n=15)$ et $8 \%$ par manque de temps $(n=4)$.

Dans $18 \%$ des réponses, elles soulignent qu'il ne s'agit pas de leur rôle $(n=5)$.

$11 \%$ des sages-femmes n'ont pas compris l'ETP $(n=6)$.

Une sage-femme pense que l'ETP n'a pas d'intérêt en PMI et une autre personne déclare que peu de femmes sont atteintes de pathologies chroniques.

\section{$\underline{4}{ }^{\text {ème }}$ partie : A propos de l'épilepsie ...}

\section{Questions 22 à 25 : Quizz sur l'épilepsie en général :}

\section{Graphique $n^{\circ} 16$}

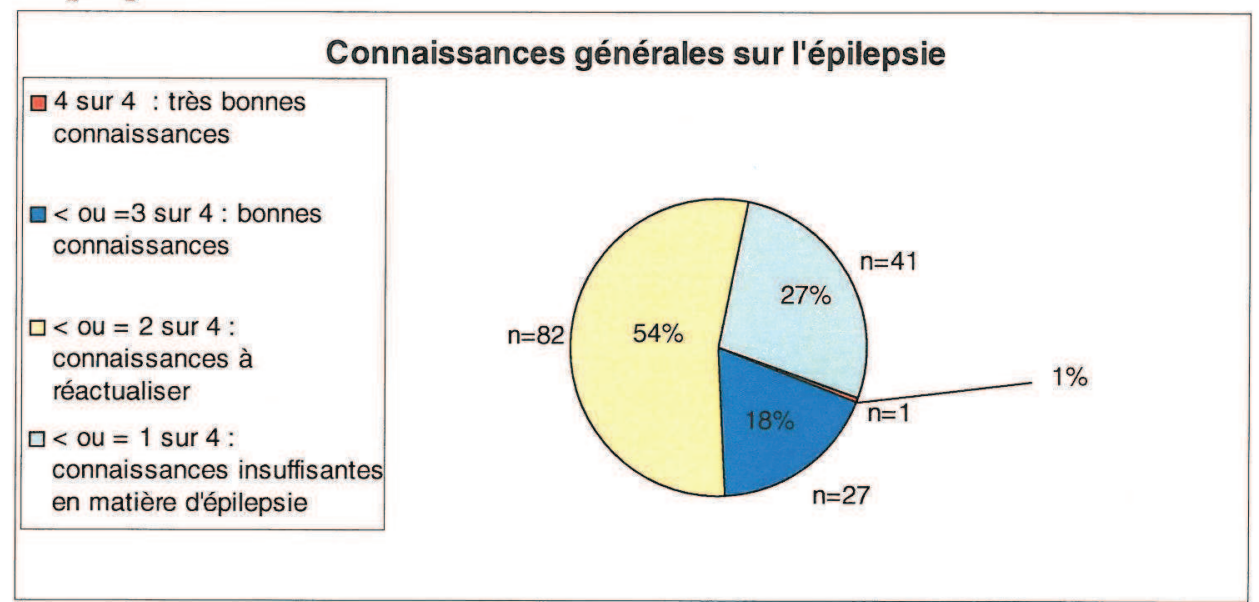

Moins d'un quart des sages-femmes a obtenu un score supérieur à 2 sur 4 .

Seul $28 \%$ de notre population a connaissance du chiffre seuil de crises épileptiques non provoquées à partir duquel nous pouvons parler d'épilepsie (question 23 : à partir de deux crises).

Pour la question 25, nous attendions cinq principales actions (cf méthodologie). $35 \%$ des sages-femmes $(\mathrm{n}=53)$ ont cité deux de ces actions.

$63 \%$ d'entre elles $(\mathrm{n}=95)$ ont cité plus de trois principales actions.

Enfin, trois sages-femmes ont utilisé les cinq actions (2\% des réponses)

Nous remarquons que huit sages-femmes de notre échantillon (5\%) ont cité la tentative d'enregistrement de l'activité cardiaque du fœtus. Nous pensons que la consigne ne devait pas être assez claire ou bien est-ce un oubli ? 


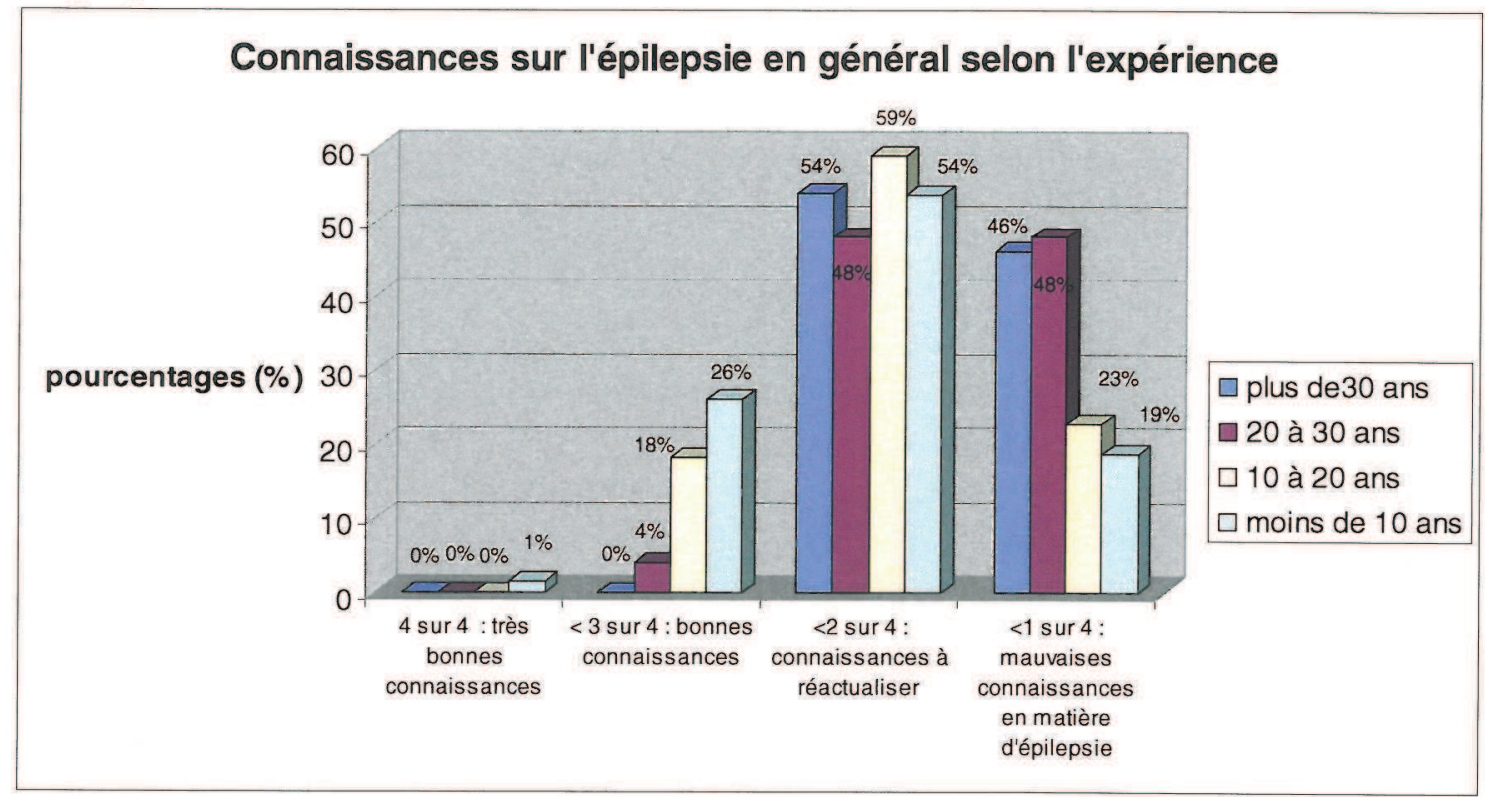

Les connaissances en matière d'épilepsie en général sont significativement meilleures chez les sages-femmes récemment diplômées (test de Fisher : p= 0.023). La sage-femme ayant obtenu un score de 4 sur 4 est diplômée depuis moins de 10 ans.

\section{Question 26: Avez-vous déjà dû prendre en charge une femme enceinte épileptique?}

$81 \%$ des sages-femmes interrogées $(\mathrm{n}=122)$ ont eu l'expérience de la prise en charge d'une femme épileptique.

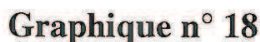

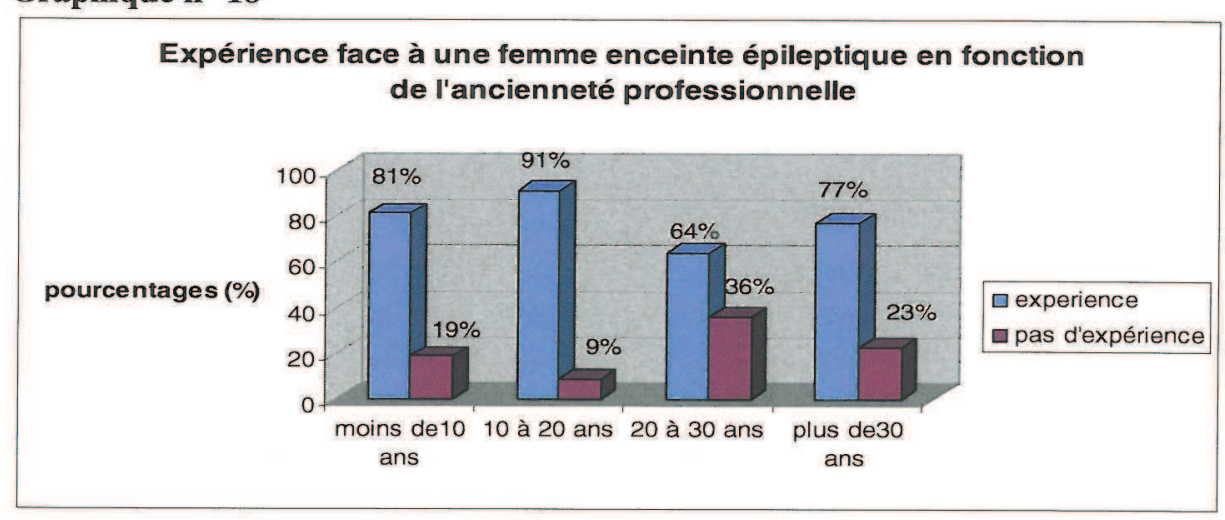

Le test de Fisher montre de manière significative $(\mathrm{p}=0.049)$ que, quelque soit leur durée d'exercice professionnel, le taux de sages-femmes ayant déjà pris en charge une femme enceinte épileptique est largement supérieur à celui des sages-femmes qui n'en ont jamais rencontrées. 


\section{Graphique ${ }^{\circ} 19$}

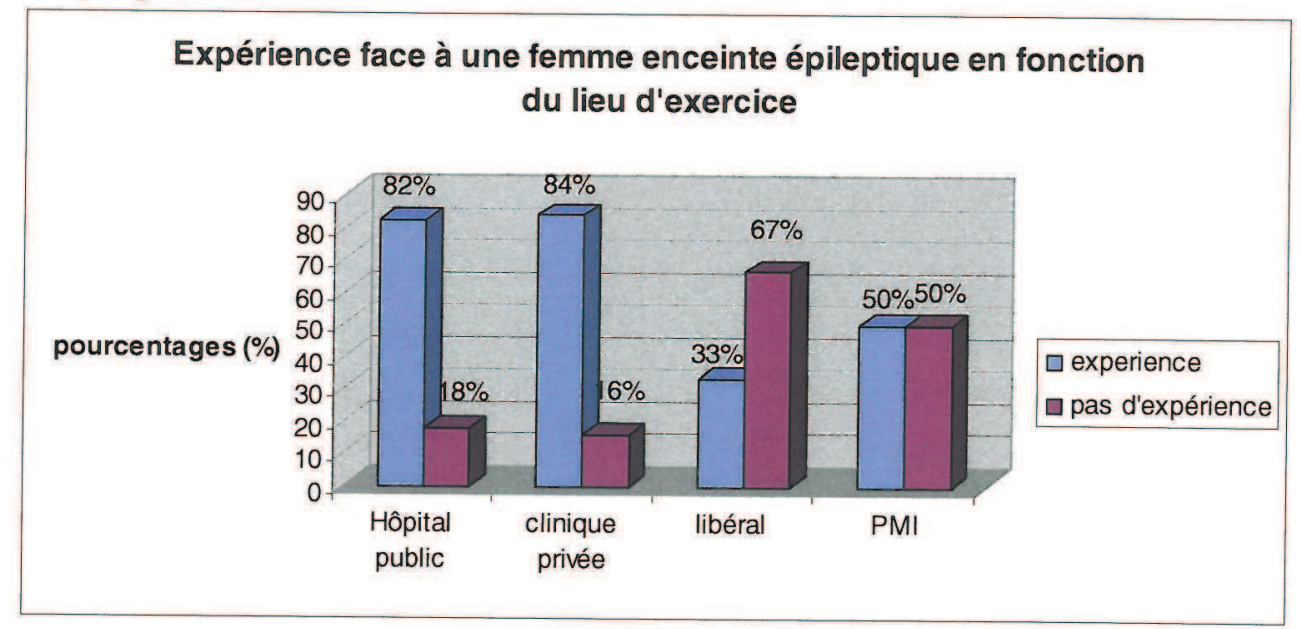

Sur ce graphique, nous constatons que l'expérience de la prise en charge de femmes épileptiques se fait plus en milieu hospitalier qu'en libéral.

En PMI, le taux de sages-femmes ayant déjà eu cette expérience est identique à celui de celles qui n'en ont jamais rencontré. Ces tendances ne peuvent être concluantes car les effectifs de sages-femmes libérales et territoriales sont insuffisants (sept questionnaires analysés au total).

\section{Question 27 : Si oui, dans quelle(s) situation(s) ?}

\section{Graphique $^{\circ} 20$}

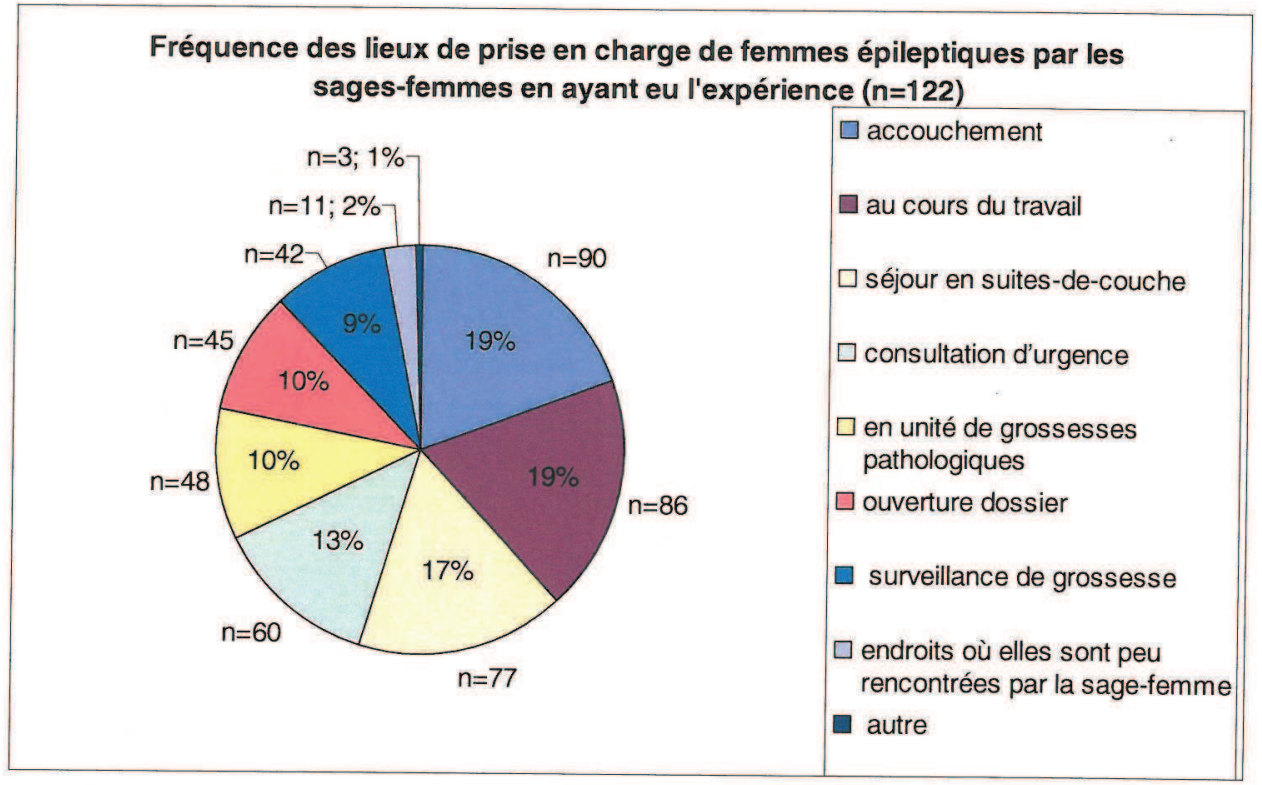

Par cette question, nous remarquons que les femmes enceintes épileptiques sont prises en charge par les sages-femmes majoritairement au bloc obstétrical (au cours du travail et à l'accouchement).

$\mathrm{Au}$ cours des consultations prénatales, les sages-femmes rencontrent fréquemment des femmes épileptiques (ouverture de dossier comme surveillance de grossesse). 
Peu de sages-femmes de notre population ont pris en charge ces patientes dans les services de diagnostic anténatal, d'échographie, planification familiale et de procréation médicalement assistée $(\mathrm{n}=3)$.

Deux sages-femmes territoriales disent avoir pris en charge une femme épileptique dans le cadre de la PMI.

Une sage-femme libérale a déjà été confrontée à cette patientèle.

Dans les réponses «autre », nous trouvons la préparation à la naissance et les visites à domicile.

\section{Question 28 : A quel(s) moyen(s) avez-vous eu recours ?}

Graphique ${ }^{\circ} 21$

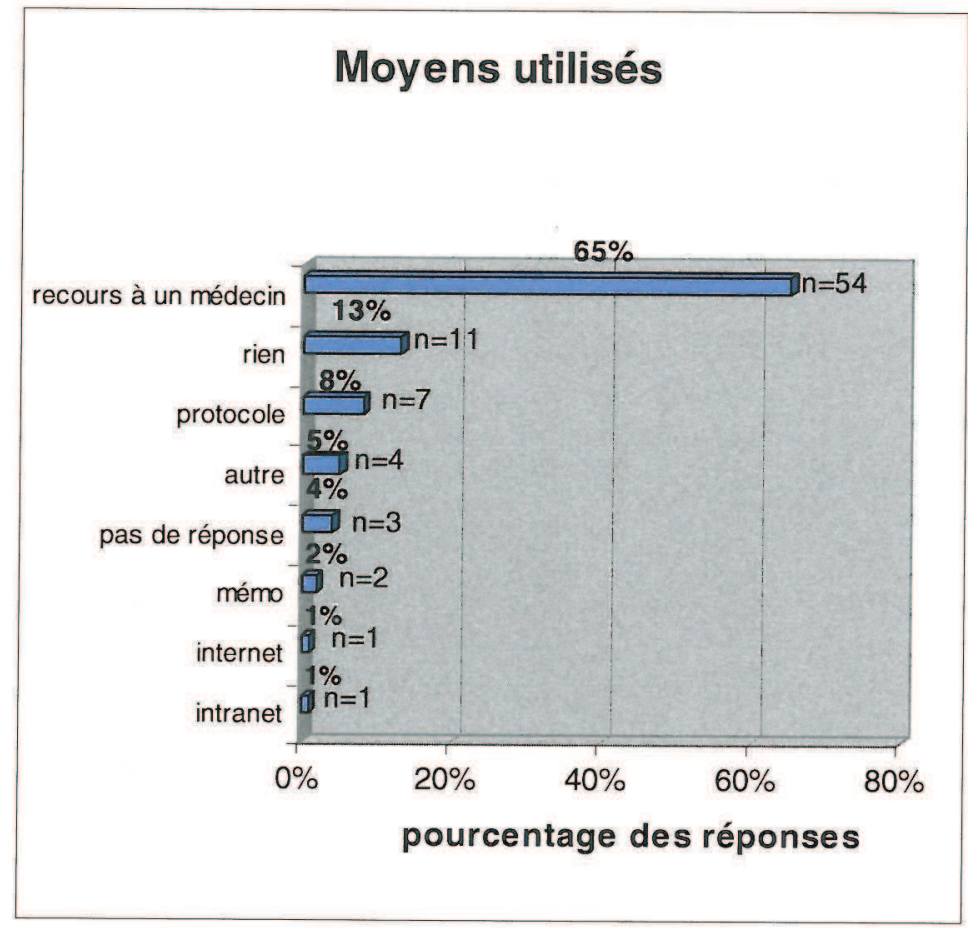

Préférentiellement (65\% des réponses), les sages-femmes font appel à un médecin. En deuxième position, notre population déclare n'avoir recours à rien ni à personne (13\%). Dans $8 \%$ des réponses, les sages-femmes ont utilisé le protocole.

30 sages-femmes (20\%) ont eu recours à plusieurs moyens. 21 d'entre elles ont associé le « recours à un médecin » et recours au « protocole». 
Graphique $n^{\circ} 22$

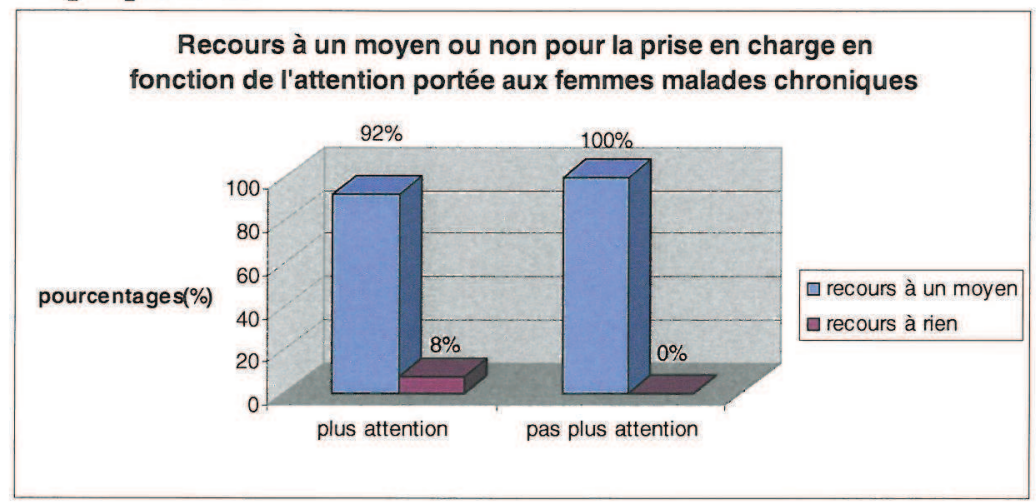

Nous constatons que les sages-femmes qui portent plus d'attention aux femmes malades chroniques, n'ont pas toujours recours à un moyen pour la prise en charge de ces patientes. Le test de Fisher est non significatif $(p=1)$.

Question 29 : Si non, quelles sont les propositions que vous associeriez à la prise en charge d'une femme enceinte épileptique si cette situation se présentait :

Graphique $n^{\circ} 23$

Qualifications données à la prise en charge d'une patiente épileptique par les sages-femmes n'ayant jamais rencontré cette situation $(n=29)$

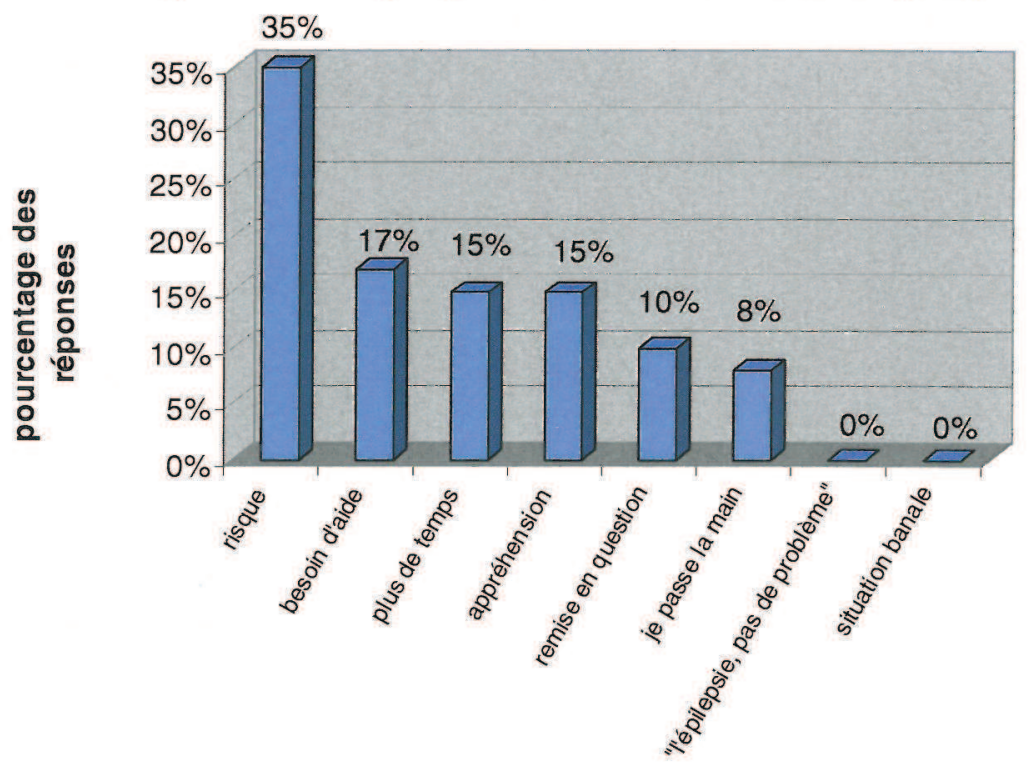

29 sages-femmes n'ont jamais rencontré de femmes enceintes épileptiques. Dans $35 \%$ des réponses, elles ont qualifié cette prise en charge comme étant à risque.

Aucune n'a retenu les propositions : «l'épilepsie, pas de problème ! » et « situation banale ». 


\section{Questions 30 à 35 : Quizz « épilepsie et grossesse »}

\section{Graphique ${ }^{\circ} 24$}

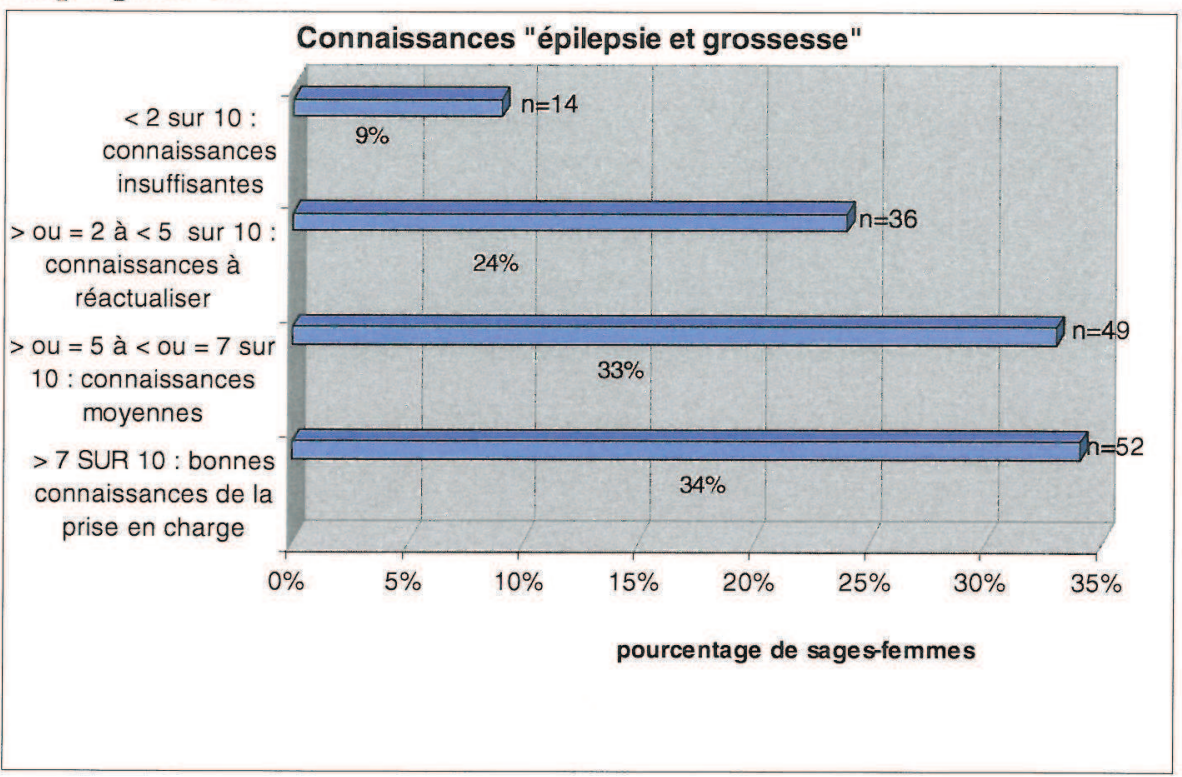

Les connaissances des sages-femmes en matière de prise en charge de patientes

épileptiques sont moyennes voire bonnes (près des deux tiers de notre échantillon).

9\% des sages-femmes interrogées ont montré des connaissances insuffisantes.

Par le biais de ce quizz, nous pouvons observer à la question 30 que la contraception d'une femme épileptique pose problème chez plus de la moitié des sagesfemmes $(56 \%)$ et notamment à cause des interactions médicamenteuses.

La question sur l'échographie supplémentaire a été majoritairement réussie (73\% de bonnes réponses) mais les raisons de cette échographie n'ont pas toujours été les bonnes (seulement $57 \%$ de réponses correctes).

Nous constatons que la question 31 portant sur les risques des femmes enceintes épileptiques pendant la grossesse a été moins réussie : 17\% des sages-femmes ont répondu correctement. Plus de la moitié $(55 \%)$ d'entre elles n'ont donné que deux bonnes réponses. $27 \%$ ont donné moins de deux réponses attendues.

A la question 33, $62 \%$ des sages-femmes savent que l'acide valproïque est contreindiqué pendant la grossesse mais seul 54\% d'entre elles en connaissent l'explication (le risque tératogène majeur). 
Graphique ${ }^{\circ} 25$

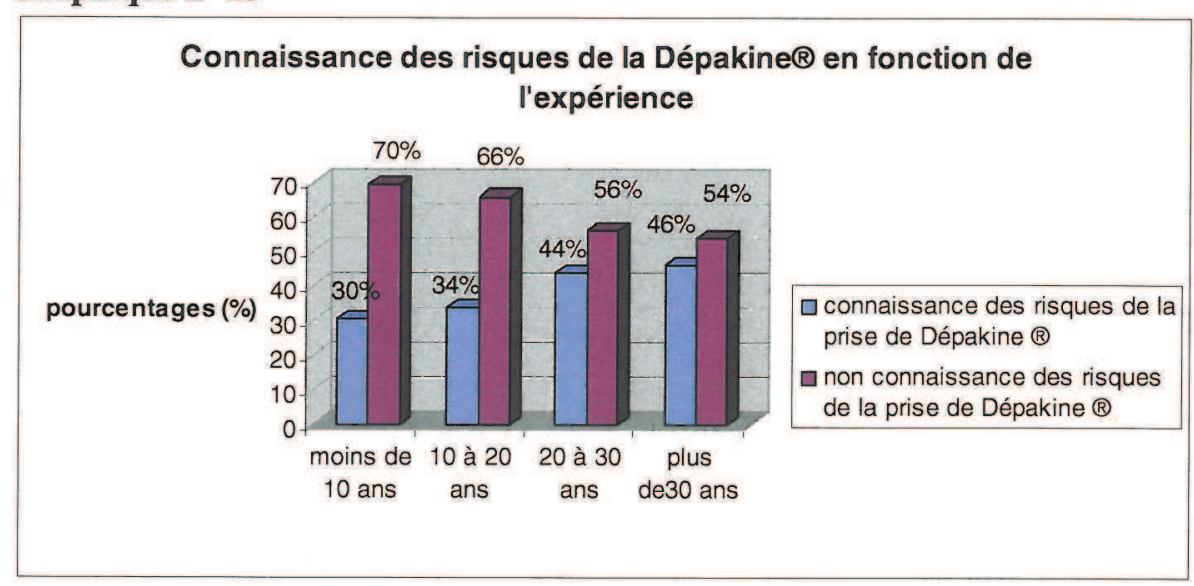

Nous constatons, de manière non significative (le test du chi2 donne un $\mathrm{p}=0.12$ ), que les recommandations sur la Dépakine @ ne sont pas connues pour plus de la moitié des sages-femmes quelque soit leur nombre d'années d'expérience.

Les sages-femmes exerçant depuis plus de 20 ans ont un taux supérieur de bonnes réponses. L'explication pourrait provenir du fait que les premiers résultats des études sur cette tératogénicité datent des années 1980.

A la question 35, seuls 5\% des sages-femmes ont su donner quatre risques potentiels d'un nouveau-né de mère épileptique. $27 \%$ d'entre elles n'ont donné aucun risque.

$>$ Question 36: Ressentez- vous le besoin d'améliorer votre gestion de ces situations?

$80 \%$ des sages-femmes souhaitent s'améliorer dans la prise en charge de ces patientes. $4 \%$ d'entre elles n'ont pas donné de réponses à cette question.

Graphique $n^{\circ} 26$

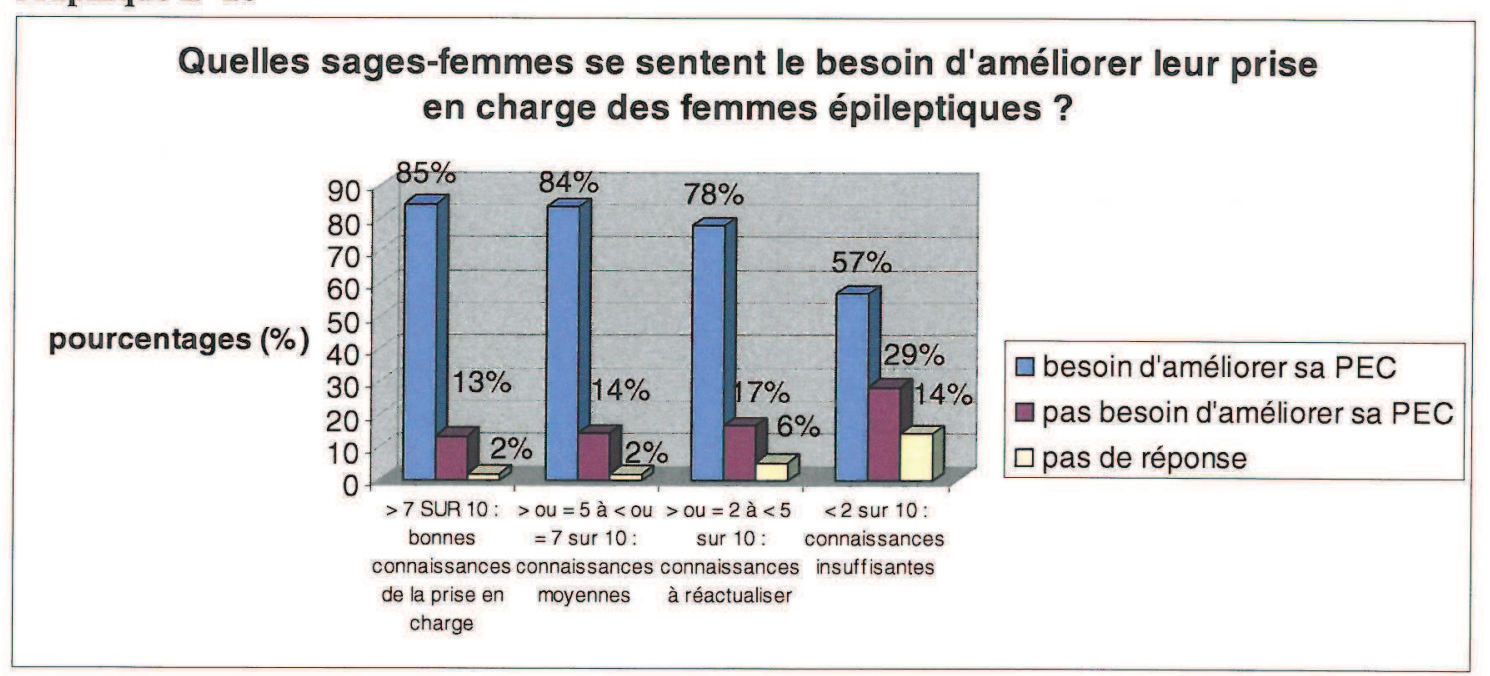

Quelque soit le score obtenu au quizz sur «épilepsie et grossesse», les sagesfemmes ressentent le besoin de s'améliorer (test de Fisher non significatif : $\mathrm{p}=0.242$ ). 
Cependant, nous remarquons que dans la catégorie des personnes ayant eu un score inférieur à 2 sur 10 ce besoin est moins fort (57\%) et 14\% de ces sages-femmes n'ont pas donné de réponses.

Question 37 : Des protocoles ou des guides sur la prise en charge des patientes épileptiques sont-ils disponibles dans les services pour que vous vous y référiez ?

$56 \%$ des sages-femmes affirment qu'il n'existe aucun protocole en matière d'épilepsie et grossesse dans leurs services. $8 \%$ ne le savent pas $(n=12)$.

Tableau $n^{\circ} 5$

\begin{tabular}{|l|c|c|c|}
\hline & Protocole & $\begin{array}{c}\text { Pas de } \\
\text { protocole }\end{array}$ & Ne sait pas \\
\hline HPE & 2 & 5 & 0 \\
\hline Dieppe & 6 & 2 & 0 \\
\hline Evreux & 2 & 13 & 1 \\
\hline CHU & 9 & 8 & 1 \\
\hline Belvédère & 16 & 2 & 2 \\
\hline Bernay & 5 & 1 & 1 \\
\hline Elbeuf & 7 & 2 & 1 \\
\hline Monod & 14 & 4 & 4 \\
\hline Mathilde & 12 & 5 & 0 \\
\hline Fécamp & 5 & 2 & 0 \\
\hline Lillebonne & 2 & 2 & 1 \\
\hline Gisors & 3 & 2 & 0 \\
\hline
\end{tabular}

Dans aucun des établissements interrogés, les sages-femmes sont en accord quant à la présence ou non d'un protocole de service.

\section{Question 38 : Avez- vous le sentiment qu'au sortir de l'école vous étiez apte à} prendre en charge une femme enceinte épileptique ?

$31 \%$ des sages-femmes interrogées se sentaient aptes à la sortie de l'école. $66 \%$ ne se sentaient pas capables et $3 \%$ de notre population n'a pas donné de réponse.

Quelque soit l'année d'obtention du diplôme, dans la majorité des réponses, les sages-femmes ne se sentaient pas aptes pour cette prise en charge à la sortie de l'école (test de Fisher significatif : $\mathrm{p}=0.013$ ).

\section{Question 39: Quels outils supplémentaires souhaiteriez-vous pour que votre} prise en charge soit optimale?

Les sages-femmes demandent en priorité des protocoles de service (19\% des réponses) et une formation continue (19\%).

La proposition de schémas thérapeutiques a été retenue dans $17 \%$ des réponses.

Dans les propositions faites par les sages-femmes, nous retrouvons le rôle que pourrait jouer le réseau périnatal. 


\section{Discussion}


La sage-femme exerce une profession médicale mais son champ de compétence est limité en cas de pathologie.

De nos jours, la prévalence des pathologies chroniques ne cesse d'augmenter. La grossesse des patientes présentant une maladie chronique relève alors du domaine de la pathologie et la sage-femme s'y trouve confrontée.

De ce fait, le but de notre enquête était d'évaluer la place de la sage-femme dans la prise en charge de ces patientes au cours de la grossesse.

\section{Le profill de la population étudiée}

En 2010, le nombre de sages-femmes en France s'élevait à 23365. On en dénombrait 488 en Seine-Maritime et 89 dans l'Eure soit 577 (59) pour ces deux départements.

Avec un retour de 151 questionnaires, notre échantillon représente un peu plus d'un quart de la population des sages-femmes de Haute Normandie. Notre étude est donc réalisée sur un faible effectif. En aucun cas, il ne peut être considéré comme représentatif d'un échantillon modèle des sages-femmes de France.

Depuis 1982, la profession de sages-femmes est accessible aux hommes. Elle se masculinise plus fortement depuis la sélection par les études de médecine en 2001. Au premier janvier 2011, le nombre d'hommes sages-femmes étaient de 7 (30) en Haute Normandie soit 1,4\%. En France, on parle de 1,7\% d'hommes.

Huit sages-femmes hommes ont répondu à notre questionnaire. Nous avons donc réussi à toucher la totalité des hommes de Seine-Maritime et de 1'Eure. Du fait de notre échantillon plus faible (151 sages-femmes), la proportion hommes-femmes est largement augmentée (5\% d'hommes) donc non représentative par rapport aux proportions régionale et nationale.

La moyenne des âges des sages-femmes que nous avons interrogées est d'un peu plus de 34 ans $(34,43$ ans), ce qui est en dessous de la moyenne nationale $\approx 42$ ans (29). Notre échantillon n'est donc pas représentatif à l'échelle nationale.

La tranche d'âge 25-30 ans a été largement représentée. Ce qui est retrouvé en termes d'ancienneté d'exercice puisque la catégorie des moins de 10 ans d'expérience professionnelle est également surreprésentée (46\%). Nous pensons que cette tendance s'explique par la part importante de sages-femmes hospitalières interrogées par rapport à celles de PMI et libérales (n=7). En effet, depuis le plan de périnatalité 1994-2000 (27) visant à des normes optimales de personnel et d'équipement, les sages-femmes 
nouvellement diplômées sont prioritairement embauchées au sein des établissements. De plus, depuis 2003, les pouvoirs publics ont régulièrement augmenté le quota d'entrée dans les écoles de sages-femmes pour faire face aux besoins : 900 places en 2002 à 1015 places en 2011 (59).

Nous émettons également l'hypothèse que les jeunes sages-femmes, dont les préoccupations étudiantes ne sont pas si lointaines, se sont senties plus investies à répondre à notre enquête.

En Haute-Normandie, en 2010, le Conseil de 1'Ordre des sages-femmes recensait un taux de $64 \%$ de sages-femmes hospitalières en établissement public, $10 \%$ en privé, 4 $\%$ d'entre elles exerçant en secteur PMI et $6 \%$ en libéral (59).

Dans notre étude, nous avons recensé respectivement $79 \%$ de sages-femmes en secteur public hospitalier, $16 \%$ en privé, $3 \%$ en PMI et $2 \%$ en libéral.

Nous pouvons donc conclure que les proportions de notre population sont assez proches et représentatives de celles de notre région, à l'exception des sages-femmes libérales (faible effectif).

En règle générale, notre questionnaire a suscité des difficultés quant à sa longueur et sa complexité. Certaines sages-femmes ont mentionné leur besoin, à la suite des questions, de réactualiser leurs connaissances.

Nous avions pensé qu'être confronté à titre personnel à une pathologie chronique aurait influencé les réponses mais, que ce soit en matière de pathologie chronique comme d'ETP, l'atteinte personnelle n'a joué aucun rôle.

\section{Les pathologies chroniques}

\subsection{Connaissances des sages=femmes}

La pathologie chronique est définie par les notions de durée, d'incurabilité, de dépendance vis-à-vis d'un traitement, d'ancienneté, d'évolution et de répercussions sur la vie quotidienne. Généralement, la notion de durée a été fortement soulignée par nos sages-femmes (dans $73 \%$ des réponses données). En revanche, elles ont peu perçu les répercussions sur la vie quotidienne (4\% des réponses). Pourtant, celles-ci sont la difficulté même des pathologies chroniques. Le diagnostic d'une pathologie chronique implique des changements d'habitude, des rapports à l'autre différents, une modification des rôles au sein de la famille, un nouvel apprentissage du malade... 
En pratique, les 2 pathologies chroniques citées majoritairement par les sagesfemmes (questions 7 comme 8) sont à l'image des chiffres mondiaux : le diabète et l'hypertension artérielle chronique.

En revanche, il est intéressant de constater que l'obésité a été très peu citée comme exemple de pathologie chronique (1.4\% des réponses) et comme pathologie fréquemment rencontrée (citée 4 fois). Elle atteint pourtant près de 300000000 de personnes dans le monde. Il s'agit d'un facteur d'évolution défavorable en obstétrique avec des risques plus élevés pour le déroulement de l'accouchement, la santé de l'enfant et de la mère (diabète gestationnel, hypertension...). On comptait, en France, 7,4 \% de femmes souffrant d'obésité en 2003 et 9,9\% en 2010 (26).

Etre malade chronique implique généralement la prise d'un traitement à vie. De nos jours, on constate des problèmes d'observance des traitements par le patient.

Cela concerne aussi bien les traitements prescrits que les comportements d'hygiène de vie des personnes impliquées : rendez-vous manqués, régime non suivi, posologie non respectée, arrêt prématuré de médicament, prescription non renouvelée.

Un certain nombre d'évaluations de l'OMS a établi que la proportion de malades chroniques respectant leur traitement n'était que de $50 \%$ dans les pays développés (52). Or, ce problème va s'amplifier à mesure que la charge mondiale des maladies chroniques va croître. Les professionnels de santé sont en première ligne pour tenter d'y remédier. Leur rôle est de dépister les facteurs pouvant diminuer l'observance de leur patient et surtout d'essayer de comprendre ce qui est difficile pour lui. Cela fait appel à toutes les capacités relationnelles du soignant (écoute active, empathie).

Les sages-femmes de notre étude pensent que les trois principaux facteurs influençant l'observance sont le déni de la maladie ( $21 \%$ des réponses), le niveau socioéconomique (20\%) et les effets secondaires des traitements (15\% des réponses).

La grossesse implique également, pour les femmes atteintes d'une pathologie chronique, une nouvelle adaptation du traitement de leur maladie. Cela peut également favoriser une mauvaise observance, notamment elles ont peur d'une atteinte pour le fœtus (soulignée par une personne dans notre étude).

Par contre, nous avons mis en évidence un paradoxe: pour nos sages-femmes, les problèmes psycho-sociaux liés à la pathologie ne sont pas des facteurs compliquant la grossesse d'une femme malade chronique alors qu'elles évoquent qu'ils favorisent la non observance. 
Ainsi, nous pensons qu'en général, les sages-femmes ont une connaissance partielle des pathologies chroniques, elles négligent les répercussions de la pathologie sur la vie quotidienne.

\subsection{L'implication des sages-femmes face aux patientes atteintes de pathologies chroniques}

En général, les sages-femmes ont répondu accorder une attention plus particulière à ces patientes ( $94 \%$ de notre population). Elles sont conscientes du caractère dangereux de leur grossesse et qu'une patiente malade chronique doit avoir un suivi médical.

Nous avons alors divisé les sages-femmes en deux catégories.

Dans un premier temps, l'analyse des réponses des sages-femmes de pmi et libérales montre un faible taux de retours de questionnaires. Il faut prendre en compte qu'elles ont été contactées par voie postale et que les questions ne s'accordaient pas toujours avec leur mode d'exercice. Elles nous ont d'ailleurs souvent fait remarquer qu'elles ne prennent pas en charge ces pathologies. Se sont-elles senties moins concernées ?

Nous savons que leur travail peut s'exercer en toute autonomie dans la physiologie. Pourtant, elles ont un rôle essentiel de coordination et d'orientation des femmes enceintes et de leur famille auprès des différents réseaux sanitaires et sociaux.

En outre, leurs missions précisent qu'elles peuvent être amenées à exercer au domicile des patientes lors de la surveillance de grossesses pathologiques (retour à domicile de patientes présentant une menace d'accouchement prématuré modérée, surveillance de la prise de traitements...).

Dans la seconde catégorie, nous avons regroupé les sages-femmes hospitalières du secteur privé et du secteur public.

L'analyse des réponses des sages-femmes des maternités de niveau 1 a montré que la physiologie est une priorité pour elles. Les patientes atteintes de pathologies chroniques ne relèvent pas de leur rôle.

Effectivement, le Plan de périnatalité 1994-2000 (27) a permis d'instaurer une typologie des maternités. Les niveaux de prise en charge ont été définis par le niveau de soin pédiatrique associé dans le même établissement afin de préserver le lien mère-enfant. Les maternités de type 1 sont habilitées à prendre en charge des grossesses sans facteur périnatal et associant des soins pédiatriques de niveaux I (surveillance de nouveau-nés bien portants en maternité) et II (nouveau-nés ayant des troubles mineurs avec possibilités de traitement auprès des mères). 
Celles de niveau 2 peuvent prendre en charge des grossesses susceptibles de donner naissance à des nouveau-nés nécessitant des soins de niveau III (nouveau-nés présentant des pathologies nécessitant une surveillance un service de pédiatrie néonatale).

Enfin, les maternités de niveau 3 doivent prendre en charge les grossesses susceptibles de donner naissance à des nouveau-nés nécessitant des soins de niveaux IV et V (soins dispensés en service de soins intensifs et de réanimation néonatale).

Nous comprenons que les sages-femmes de niveau 1 aient eu des difficultés en ce qui concerne notre sujet. Les grossesses des femmes atteintes de pathologies chroniques sont considérées comme étant « à risque » pour la mère et dans la majorité des cas pour l'enfant.

Pourtant, l'analyse de notre questionnaire a montré que parmi nos 24 sages-femmes travaillant en niveau 1, 18 d'entre elles (75\%) ont déclaré avoir eu l'expérience de prendre en charge une femme épileptique et $40 \%$ d'entre elles déclarent qu'un protocole en matière d'épilepsie est présent au sein des services.

Ces pourcentages sont élevés pour une prise en charge de grossesse pathologique.

Les recommandations de la HAS de 2007 quant au suivi et l'orientation des femmes enceintes en fonction des situations à risque identifiées (ANNEXE XI) nous permettent de comprendre ces taux. En effet, certaines pathologies chroniques peuvent avoir un suivi régulier par les sages-femmes et le choix de l'établissement est à adapter en fonction des risques.

En réalité, la sage-femme prend forcément en charge, à un moment de leur grossesse, une de ces patientes. Les premiers résultats de l'enquête nationale périnatale de 2010 précisent :

«En cas d'accouchement par voie basse, $80 \%$ des femmes mentionnent que 1'accouchement est réalisé par une sage-femme (92 \% en hôpital public et $55 \%$ en établissement privé) » (26).

Etre atteinte d'une pathologie chronique ne signifie pas avoir obligatoirement une césarienne. Ainsi, la prise en charge, au bloc obstétrical, pendant le travail et l'accouchement se fait par la sage-femme. Dans notre enquête, par exemple, 38\% des prises en charge de patientes épileptiques par les sages-femmes se sont faites au bloc obstétrical.

Au moment de l'ouverture du dossier obstétrical, la sage-femme joue également un rôle primordial. En effet, la consultation pré-conceptionnelle n'étant pas encore ancrée dans les mœurs, les sages-femmes de consultation, aux mêmes titres que les médecins 
généralistes et les gynécologues médicaux (tous trois acteurs de soins de santé primaires) ont la responsabilité d'évaluer le niveau de risque des femmes et d'en référer, en cas de présence ou d'apparition de facteurs de risque, auprès des gynécologuesobstétriciens (acteurs de soins de sante secondaires).

Par ailleurs, seules 4 sages-femmes (3\% de notre population) ont déclaré accorder autant d'attention aux femmes enceintes atteintes de pathologies chroniques qu'aux femmes «sans problème de santé ». Effectivement, la grossesse n'est pas une maladie mais il s'agit d'un bouleversement à la fois d'ordre physique et psychologique pour toute femme. Ça l'est d'autant plus pour les femmes malades chroniques.

Notre première hypothèse semble confïmée: les sages-femmes se sentent impliquées, malgré la pathologie, face aux patientes malades chroniques.

\section{L'éducation thérapeutique}

\subsection{Connaissances des sages-femmes}

L'éducation thérapeutique du patient gagne progressivement les corps de métier de la santé. En revanche, les sages-femmes semblent peu informées que l'ETP entre tout à fait dans leurs compétences.

Beaucoup de sages-femmes ont «entendu parler» de l'ETP (89\% dans notre enquête). Les services de diabétologie ont été fortement mentionnés dans ce cadre. Nous pouvons considérer qu'ils font partie, pour la majorité d'entre elles, des expériences de stage au cours de la formation sage-femme. D'ailleurs, nous constatons que cette discipline est rencontrée dans $26 \%$ des cas grâce à notre formation (études et terrains de stage).

Cependant, comme nous l'avons vu, contrairement aux professionnels de santé tels que les infirmiers, les sages-femmes n'ont pas une formation initiale en ETP dans leur cursus. Elles en sont informées de manière transversale à travers différents cours (santé publique notamment).

C'est pourquoi, on observe une certaine méconnaissance de la définition de ce concept. Nous constatons, dans un premier temps, que $7 \%$ de nos sages-femmes n'ont pas su donner de définition. 16\% d'entre elles ont confondu l'ETP avec le fait de transmettre une information, une explication au patient.

Ensuite, en analysant plus particulièrement les bonnes réponses, une remarque essentielle doit être faite: une seule sage-femme a explicité que cette discipline est 
utilisée dans le cadre de la prise en charge des pathologies chroniques. Il s'agit là de sa définition première.

En outre, $80 \%$ des sages-femmes pensent faire de l'éducation thérapeutique dans leur profession. Mais en réalité, seulement dans $41 \%$ des cas, il s'agit véritablement d'ETP (prise en charge de femmes diabétiques, de pathologies chroniques, adaptation de traitement pendant la grossesse). Pour les $59 \%$ des justifications restantes, il s'agit de l'éducation à la santé (contraception, nutrition, hygiène de vie, rééducation périnéale...).

Une partie de notre $2^{\text {ème }}$ hypothèse est confirmée, les sages-femmes méconnaissent le concept de 1'ETP.

Les sages-femmes semblent savoir que l'ETP leur est accessible : 90\% d'entre elles ont répondu que la sage-femme peut s'inscrire dans un programme d'ETP. Mais elles ne souhaitent pas forcément se former. $63 \%$ des sages-femmes interrogées ont montré un intérêt pour une formation en ETP. Une seule d'entre elles est déjà formée.

Un tiers d'entre elles souhaiterait être plus à l'aise avec les patientes malades chroniques et aimerait mieux les encadrer. Là encore, le terme est mal choisi. Cela provient sans doute toujours du flou de la définition des pathologies chroniques. Les professionnels de santé devraient plutôt parler de s'adapter au patient.

L'enrichissement de leurs connaissances et la formation continue constituent leur deuxième motivation. Cette réponse est intéressante compte tenu du changement actuel en matière de formation continue : le Développement Professionnel Continu (71) (72). Les sages-femmes ont désormais l'obligation annuelle de participer à un programme de développement professionnel continu.

La formation à $l^{\prime} E T P$ pourrait avoir un essor dans les prochaines années car elle se «marie » parfaitement avec la politique actuelle de la prise en charge des pathologies chroniques.

Pourtant, les sages-femmes sont peu au courant des formations existantes.

Elles n'ont pas connaissance qu'il existe, initialement, une formation minimale obligatoire de 40 heures. Seul un peu plus de la moitié des sages-femmes (53\%) ont répondu qu'il existe une formation en matière d'ETP. Près des deux tiers de notre population ne connaissent pas les formations d'ETP. 7\% des sages-femmes ont pensé à un diplôme universitaire (DU) d'ETP mais aucune d'entre elles ne connait le master en ETP. Pourtant, 1'université de Rouen est une des rares à le proposer. 
Un petit nombre de sages-femmes (7\% de notre population) a pensé au DU de pathologies maternelles et grossesse. Cette réponse est intéressante car elle souligne que les sages-femmes pensent d'abord à se former en matière de pathologie avant même d'éducation.

Les sages-femmes savent qu'elles peuvent être actrices de l'ETP mais méconnaissent les formations qui leur sont proposées.

\subsection{L'ETP au cours de la grossesse}

La sage-femme occupe une place importante auprès des patientes. Sa dimension relationnelle est un atout dans la prise en charge globale des femmes enceintes.

Actuellement, les patientes présentant un diabète insulinodépendant pendant la grossesse (diabète préexistant à la grossesse et diabète gestationnel) sont orientées vers un service de diabétologie afin d'être éduquées à l'insulinothérapie et au régime diabétique. Les sages-femmes sont écartées de 1'éducation thérapeutique.

Ainsi, il nous tenait à cœur de faire une proposition aux sages-femmes : inclure dans le suivi des femmes enceintes des actions d'ETP. $81 \%$ des sages-femmes se sont montrées favorables à cette proposition.

Elles ont d'ailleurs valorisé, dans leurs réponses, leur métier et leur place auprès des patientes (prise en charge globale de la patiente, plus de temps auprès des patientes, rôle d'accompagnement...).

La grossesse est un moment clé pour l'éducation des femmes en général. Elles sont plus motivées et réceptives, notamment pour le bien-être de leur futur enfant.

C'est pourquoi certaines sages-femmes (5\%) ont souligné que la grossesse serait un moment préférentiel pour mettre en place ce type de programme.

\section{L'exemple de l'épilepsie}

Dans la littérature, on retrouve toujours la même conclusion : 1a prise en charge des femmes épileptiques au cours de la grossesse est à améliorer (une meilleure information et planification de leur grossesse, une collaboration plus étroite entre les professionnels avec un discours plus cohérent et une plus grande information quant aux traitements).

\subsection{Connaissances des sages-femmes}

Du point de vue des connaissances générales, les scores des sages-femmes montrent que les connaissances de notre population sont à réactualiser (54\%). Nous avons conscience que les questions étaient difficiles car la neurologie n'est pas leur spécialité. 
Les meilleures connaissances en matière d'épilepsie reviennent aux sages-femmes récemment diplômées car sans doute plus récentes dans leur esprit.

Parmi les questions, nous pouvons tout de même regretter que la valeur seuil de crises épileptiques non provoquées à partir de laquelle on peut parler d'épilepsie est peu connue ( $28 \%$ de bonnes réponses).

En outre, les sages-femmes ne connaissent pas parfaitement les gestes d'urgence à appliquer face à une patiente en crise. 3 d'entre elles seulement ont évoqué les 5 principales actions à effectuer. Pourtant, il s'agit des mêmes gestes qu'en cas de crise d'éclampsie (excepté la drogue utilisée).

Huit sages-femmes seulement ont pensé à la tentative d'enregistrement des bruits du cour fotal. Ce chiffre est insuffisant compte-tenu du risque d'hypoxie du foetus au cours d'une crise même si déontologiquement la sécurité de la mère passe avant tout.

En ce qui concerne l'association «épilepsie et grossesse », les connaissances des sages-femmes sont moyennes voire bonnes (67\% des sages-femmes ont entre 5 et 10 sur 10) mais non optimales. Bien évidemment, la sage-femme n'a pas la prise en charge exclusive de la femme enceinte épileptique mais il est important qu'elle ait des connaissances suffisantes pour pouvoir informer ses patientes ou tout du moins les orienter au mieux (importance de la contraception, la supplémentation, l'échographie supplémentaire...).

Dans la question 33 de notre questionnaire, 38\% de nos sages-femmes n'ont pas été alertées par une grossesse sous Dépakine ${ }^{\circledR}$, le traitement le plus tératogène. Pourtant, «Les actions de tous les professionnels de santé concernés (gynécologues, neurologues, sages-femmes, généralistes...) doivent se conjuguer pour éviter la survenue d'une grossesse sous acide valproïque sans réévaluation de la situation avant la conception $»(10)$. De plus, dans le cadre de la formation continue, les sages-femmes devraient avoir pris connaissance de cette contre-indication.

Il existe également des risques non négligeables pour le nouveau-né de femmes épileptiques. En l'occurrence, le syndrome de sevrage, la léthargie, l'apparition de convulsions peuvent être dépistés en suites-de-couches. Il serait important que les sages-femmes les connaissent.

\subsection{Des moyens supplémentaires pour la prise en charge de ces femmes}

Nous pouvons noter que $66 \%$ des sages-femmes de notre enquête ne se sentaient pas aptes, à la sortie de l'école de sage-femme, à prendre en charge une femme enceinte épileptique. 
L'épilepsie est encore une pathologie stigmatisée et impressionnante. Les sagesfemmes n'ayant jamais rencontré de femmes enceintes épileptiques qualifient cette prise en charge comme étant à risque et dans $15 \%$ des réponses, nous retrouvons une certaine appréhension de cette situation.

$65 \%$ des sages-femmes font appel à un médecin lorsqu'elles rencontrent une patiente épileptique. $8 \%$ d'entre elles se réfèrent à un protocole. Ce faible pourcentage est expliqué par le fait qu'il subsiste un manque réel de protocole au sein des services $(56 \%$ des sages-femmes affirment qu'il n'en existe aucun et $8 \%$ d'entre elles ne le savent pas) ou bien ils sont mal accessibles (dans aucun des établissements interrogés, les sages-femmes sont en accord quant à leur présence ou non).

C'est pourquoi elles demandent en priorité des protocoles de service et une formation continue pour améliorer leur pratique (38\% des réponses).

Nous pouvons affirmer notre troisième hypothèse : les sages-femmes n'ont pas les moyens nécessaires et suffisants pour assurer ces prises en charge en terme de connaissances initiales et continues mais aussi du point de vue des aides qui leurs sont proposées.

\section{La place de la sage-femme dans la prise en charge des patientes atteintes de} pathologies chroniques pendant la grossesse : propositions d'action

L'activité des sages-femmes s'est considérablement modifiée au cours de ces dernières années. La formation évolue. Les capacités de diagnostic ont nettement progressé, bouleversant la perception de la grossesse normale. La participation des sages-femmes au suivi et au traitement des grossesses pathologiques est de plus en plus importante. Ainsi, les sages-femmes ont une place dans la prise en charge de ces patientes mais actuellement, elle n'est pas vraiment définie.

Les sages-femmes perçoivent bien la complexité de la grossesse pour les femmes malades chroniques et notamment la multiplicité des professionnels intervenant auprès d'elles. En outre, la coordination interprofessionnelle est encore insuffisante. D'autant plus, lorsque des facteurs psycho-sociaux se surajoutent (on observe une surveillance prénatale tardive plus fréquente et une moins bonne observance des traitements chez les femmes vivant dans un contexte social difficile).

Nous avons réfléchi alors à des propositions d'actions : 


\section{BIBLIOGRAPHIE}

\section{Ouvrages:}

(1) JAMES - Neuro-anatomie $3^{\text {ème }}$ édition - Edition de boeck -2006

(2) Lacroix et Assal - l'éducation thérapeutique des patients Accompagner les patients avec une maladie chronique : nouvelles approches. $3^{\text {ème }}$ édition revue et complétée

(3) LANSAC, MAGNIN - Obstétrique $5^{\text {ème }}$ édition pour le praticien - Issy-lesMoulineaux- Edition MASSON - 2008 - pages 205 à 208

(4) MARPEAU- Traité d'obstétrique - Issy-les-Moulineaux- Edition MASSON 2010 - pages 61-62, pages $317-318$

(5) NGUYEN THE TICH - Exposés didactiques, Epilepsie et grossesse : le point sur le devenir des enfants de mères traitées avec les nouveaux antiépileptiques $39^{\text {èmes }}$ Journées nationales de la Société de Médecine prénatale - Angers Edition ARNETTE - 14, 15 et 16 octobre 2009 - pages 245 à 241.

(6) PERKIN - Neurologie Manuel et Atlas - Edition De boeck - 2002 - pages 47 à 56

(7) SIMON, TRAYNARD, BOURDILLON, GRIMALDI - $\quad$ Education thérapeutique Prévention et maladies chroniques - Issy-les-moulineaux - Edition Masson -2007 - pages 177 à 183 et 260 à 268.

\section{Articles:}

(8) ARTAMA, ISOJARVI, RAITANEN, and A. AUVINEN - Birth Rate among Patients with Epilepsy: A Nationwide Population-based Cohort Study in Finland "AMERICAN JOURNAL OF EPIDEMIOLOGY" - 2004 - 157(11) - 1057 à 1063. 
(9) DUPONT, BIRABEN, CRESPEL ET AL. - Epilepsies «LA REVUE DU $\underline{\text { PRATICIEN } »-2005-55-249 \text { à } 300 .}$

E. ELEFANT , F. ASSARI, M.-P. COURNOT, C. VAUZELLEGARDIER - Antiépileptiques et grossesse - Mises à jour en Gynécologie et Obstétrique - Tome XXXI - publié le 12.12.2007 - TRENTE ET UNIÈMES JOURNÉES NATIONALES - Paris, 2007 - COLLÈGE NATIONAL DES GYNÉCOLOGUES ET OBSTÉTRICIENS FRANÇAIS - Pages 73 à93

EURAP EPILEPSY PREGNANCY REGISTRY Seizure Control and Treatment in Pregnancy: "NEUROLOGY" - 2006 - 66 - 354 à 360.

HERITIER BARRAS, CHAMBOULEYRON - Patients et soignants face à l'épilepsie - Revue de la Société d'Education Thérapeutique Européenne -2010 - 2 (1) - pages 39 à 46.

HOCHLAND \& HOCHLAND HALE, CHESHIRE - Midwifery basics : caring for women with medical conditions. Neurological conditions during pregnancy and birth "PRACTISING MIDWIFE" - 2009 - 12 - 44 à 49.

HUARD, SCHALLER - Améliorer la prise en charge des pathologies chroniques Pratiques et Organisation des Soins - juillet-septembre 2010 - 41 (3) - pages 237 à 254

ITALIAN LEAGUE AGAINST EPILEPSY - Italian Consensus Conference on Epilepsy and Pregnancy, Labor and Puerperium "EPILEPSIA" $2009-50($ suppl 1) - 7 à 23.

KDOUS, FERCHIOU, HACHIDA, CHAKER, ZHIOUA, MERIAH Epilepsy and pregnancy: necessity of a combined care "LA TUNISIE MEDICALE" - 2004 - 82 - 662 à 667.

LURON- Plaidoyer pour une consultation préconceptionnelle- Brèves du CNGOF- 2007-14- article en éditorial page 1 
Éducation thérapeutique : la valeur ajoutée à la prise en charge maternofotale du diabète gestationnel (DG) La revue Sage-femme 2004 - 3 (5) - pages 204 à 209

OMS - - Journal LeParisien- 26 août 2010- rubrique santé- Epilepsie : l'OMS réclame une amélioration de l'accès aux soins en Europe

REPORT OF THE QUALITY STANDARDS SUBCOMMITEE AND THERAPEUTICS AND TECHNOLOGY ASSESMENT SUBCOMMITEE OF THE AMERICAN ACADEMY OF NEUROLOGY AND THE AMERICAN EPILEPSY SOCIETY- Management issues for women with epilepsy-Focus on pregnancy (an evidence-based review): I. Obstetrical complications and change in seizure frequency "EPILEPSIA" - 2009 - 50(5) - 1229 à 1236.

SECTION OF ELECTROENCEPHALOGRAPHY DEPARTMENT OF NEUROLOGY, SREE CHITRA TIRUNAL, INSTITUTE FOR MEDICAL SCIENCES AND TECHNOLOGY, TRIVANDRUM, INDIA - Pseudoseizures versus epileptic seizures in pregnancy "THE LANCET" - 2000 - 355 - page 318.

\section{Autres documents publiés:}

(22) Protocole du CHU de Rouen du Dr Brossard - $\underline{\text { Nouveau-né de mère }}$ épileptique- Mars 2003

GUIDE MÉTHODOLOGIQUE Structuration d'un programme d'éducation thérapeutique du patient_dans le champ des maladies chroniques Juin 2007-HAS et INPES

(24) Guide - affection de longue durée La prise en charge de votre épilepsie. Vivre avec une épilepsie sévère- HAS- novembre 2007- pages 1 à 8

(25) Guide - affection de longue durée Epilepsies graves- HAS- novembre 2007- pages 1 à 26 
Etude et Résultats Premiers résultats de l'enquête périnatale de la DREES, $\mathrm{n}^{\circ} 775 \mathrm{La}$ situation périnatale en France en 2010 et $\mathrm{n}^{\circ} 776$ Les Maternités en 2010 - octobre 2011

La sécurité et la qualité de la grossesse et de la naissance : Pour un nouveau plan périnatalité - Ministère des Affaires sociales, de la Santé et de la Ville, Haut Comité de la Santé Publique - ÉDITIONS ENSP -1994 Rennes

$\underline{\text { PLAN «périnatalité » 2005-2007 Humanité, proximité, sécurité, qualité }}$ - Ministère de la Santé - Novembre 2004

(29) Les sages-femmes en France - Rapport de recherche - Philippe Charier Université de Lyon - Janvier 2011

(30) Les professionnels de santé au $1^{\text {er }}$ janvier 2011 - Séries statistiques Daniel SICART - publié par la DREES - n 158 - juillet 2011

(31) Grossesses à risque : orientation des femmes enceintes entre les maternités en vue de l'accouchement - Argumentaire de l'HAS - Décembre 2009

$\underline{\text { Suivi et orientation des femmes enceintes en fonction des situations à }}$ risque identifiées - Recommandations de 1'HAS - Mai 2007

\section{Documents non publiés:}

Mémoires :

AISSAOUI (Djamila) - Epilepsie et grossesse - Mémoire sage-femme Rouen - 2000 .

MARTIN (Eloïse) - Prise en charge de la grossesse chez la femme épileptique - Mémoire de sage-femme - Poitiers - 2008.

MOSCA (Caroline) - La grossesse et l'accouchement des femmes épileptiques - Mémoire de sage-femme - Bordeaux - 2002.

MORIN (Charlotte) - Le vécu des sages-femmes face à la mort en périnatalité : enquête auprès de 123 sages-femmes - Rouen - 2010. 

nouvelles compétences attribuées par la loi HPST du 21 juillet 2009 - Rouen 2010.

$$
\text { FELIX (Marie) - Grossesse, moment privilégié d'informations, }
$$
d'éducation et de prévention nutritionnelles : rôle de la sage-femme - Rouen 2008.

NICOLLE (Elise) - Le déni de grossesse : enquête auprès de 169 sagesfemmes - Rouen - 2011.

Thèses :

(40) N'DIAYE (Titia) - Epilepsie et grossesse - Thèse d'exercice de médecine - Poitiers - 2008.

\section{Autres sources:}

Sites internet:

(41) http://www.vulgaris-medical.com/encyclopedie/-pilepsie-generalites7079.html (septembre 2010)

(42) http://www.fondation-epilepsie.fr/ consulté régulièrement

(43) Site du Collège National des Obstétriciens et Gynécologues français: http://www.cngof.asso.fr 31 èmes Journées nationales du CNGOF du 14 décembre 2007 - conférence de M.P. COURNOT - Anti-épileptiques et grossesse - consulté en décembre 2010.

(44) http://www.obesite-sante.com/obesite-chiffre-france-monde.php

(45) http://www.eurekalert.org/pub releases ml/2009-09/aaft-t 1091409.php

(46) http://www.sep-info.fr/fr FR/members/sclerose-en-plaques/chiffressep/index.jsp

(47) http://www.commentguerir.com/epilepsie/grossesse

(48) www.au-coeur-de-la-prevention.fr/index.php/2008/07/08/epilepsie-halteaux-idees-recues

consultés en février 2011. 
Site du Réseau science et développement :

http://www.scidev.net/fr/health/maladies-chroniques-1/features/- article Maladies chroniques : faits et chiffres - Priya Shetty - 23 juillet 2008

$$
\text { glossaire multilangue de la Banque de données en santé publique : }
$$

www.bdsp.tm.fr

Site de l'assurance maladie :

http://www.ameli.fr/professionnels-de-sante/medecins/exercer-au-quotidien/lesaffections-de-longue-duree/qu-est-ce-qu-une-affection-de-longue-duree/les-aldexonerantes.php

$$
\text { Sites de l'OMS : }
$$

$>$ http://www.who.int/mediacentre/factsheets/fs $999 / \mathrm{fr} /$

$>$ http://www.who.int/mediacentre/news/releases/2003/pr54/fr/ Communiqués de presse 2003

$>$ http://www.who.int/topics/chronic diseases/fr/chiffres épidémiologiques

$>$ http://www.who.int/whosis/whostat/FR WHS2011_Full.pdf Statistiques sanitaires mondiales 2011 Adapté des Mythes sur les Maladies Chroniques The Oxford Health Alliance, avec des informations supplémentaires tirées du supplément de The Lancet sur les maladies chroniques (Décembre 2007)

$>$ http://www.who.int/nmh/publications/ncd report-summary fr.pdf

Rapport sur la situation mondiale des maladies non transmissibles 2010. Résumé d'orientation, Organisation mondiale de la Santé 2011.

$$
\begin{aligned}
& \text { Site de l'ONU : } \underline{\text { http://www.un.org/fr/ga/ncdmeeting2011/index.shtml }} \\
& \text { http://www.un.org/apps/newsFr/storyF.asp?NewsID=26337 }
\end{aligned}
$$

(54) Site du C.R.A.T: recherches sur les traitements antiépileptiques http://www.lecrat.org/articleSearch.php3?id groupe $=15$ http://educapic.com/educ the formations.html\#100019841

$$
\text { http://www.fondationroche.org/portal/eipf/france/fondation/fondationroc }
$$

he/definition maladie chronique 
http://www.ordre-sagesfermmes.fr/NET/fr/document/2/menu/les

statistiques/nombre des sagesfemmes par departement/index.htm

http://www.ordre-sages-

femmes.fr/NET/fr/document/2/exercice de la profession/la profession et mod es dexercice/la sagefemme de pmi/index.htm

(60) http://www.ipcem.org/FORUMCl

(61) http://www.medecine.u-

psud.fr/ resources/Ressources\%2520FMC/DU/Pathologies-maternelles-etgrossesse.pdf?download=true

l'ensemble de ces sites, (49) à (61), ont été consultés de mai 2011 à février 2012

\section{$\underline{\text { Cours : }}$}

(62) Cours de PCEM1 du professeur Czernichow

(63) Cours de Pharmacologie de madame Donnadieu- Médicaments et grossesse -2011

(64) Cours de pédiatrie du Dr Lardennois NOUVEAU NE AFFECTE PAR UNE PATHOLOGIE MATERNELLE - décembre 2010

Articles de lois :

l'article L. 322-3 du Code de la Sécurité Sociale

Décret $n^{\circ}$ 2010-904 du 2 août 2010 relatif aux conditions d'autorisation des programmes d'éducation thérapeutique du patient paru dans le JOURNAL OFFICIEL DE LA REPUBLIQUE FRANCAISE le 4 août 2010- texte 25 sur 128- fait par le Premier ministre François Fillon, la ministre de la santé et des 
sports Roselyne Bachelot-Narquin, les ministres de l'intérieur, de l'outre-mer et des collectivités territoriales Brice Hortefeux et Marie-Luce Penchard.

Arrêté du 2 août 2010 relatif au cahier des charges des programmes d'éducation thérapeutique du patient et à la composition du dossier de demande et de leur autorisation de la ministre de la santé et des sports Roselyne BachelotNarquin.

Décret n²010-906 du 2 août 2010 relatif aux compétences requises pour dispenser l'éducation thérapeutique du patient paru dans le JOURNAL OFFICIEL DE LA REPUBLIQUE FRANCAISE le 4 août 2010- texte 27 sur 128- fait par le Premier ministre François Fillon, la ministre de la santé et des sports Roselyne Bachelot-Narquin, les ministres de l'intérieur, de l'outre-mer et des collectivités territoriales Brice Hortefeux et Marie-Luce Penchard.

Arrêté du 2 août 2010 relatif aux compétences requises pour dispenser l'éducation thérapeutique du patient de la ministre de la santé et des sports Roselyne Bachelot-Narquin.

Code de déontologie des sages-femmes : articles repris du Code de la Santé Publique sur les compétences générales des sages-femmes: Articles L.4151-1 ( $1^{\text {er }}$ et $3^{\text {ème }}$ alinéas) et L.4151-3.

Décret $n^{\circ}$ 2011-2117 du 30 décembre 2011 relatif au développement professionnel continu des sages-femmes paru dans le JOURNAL OFFICIEL DE LA REPUBLIQUE FRANCAISE le $1^{\text {er }}$ janvier 2012 - texte 19 sur 44 - fait par le Premier ministre François Fillon, le ministre du travail, de l'emploi et de la santé, Xavier Bertrand et le le ministre de la défense et des anciens combattants, Gérard Longuet.

Décret $n^{\circ}$ 2012-27 du 9 janvier 2012 relatif à la commission scientifique du Haut Conseil des professions paramédicales paru dans le JOURNAL OFFICIEL DE LA REPUBLIQUE FRANCAISE le 11 janvier 2012 - texte 13 sur 120 - fait par le Premier ministre François Fillon et le ministre du travail, de l'emploi et de la santé, Xavier Bertrand. 


\section{Sommaire des annexes:}

ANNEXE I : Les ALD

ANNEXE II : Les comportements face à l'annonce d'une pathologie chronique

ANNEXE III : Les compétences en ETP

ANNEXE IV : Les crises d'épilepsie

ANNEXE V : Les antiépileptiques

ANNEXE VI : Tératogénicité des antiépileptiques

ANNEXE VII : Recommandations pour la prise en charge d'une femme enceinte épileptique

ANNEXE VIII : Antiépileptiques et contraception

ANNEXE IX : Conduite à tenir en cas de crise chez la femme enceinte

ANNEXE X : Questionnaire

ANNEXE XI : Recommandations de l'HAS 2007 sur l'orientation des femmes enceintes

ANNEXE XII : Exemple de schéma thérapeutique 
- ALD30: environ 400 pathologies différentes regroupées dans 30 ALD (nombre fixé depuis le 31 décembre 1986 et dont la réactualisation dépend de la Haute Autorité de santé depuis la loi du 13 août 2004 portant sur la réforme de l'assurance maladie;

- $\quad$ ALD31 : affections « hors liste » (affections graves, de forme évolutive ou invalidante et reconnues par le contrôle médical, ne figurant pas sur la liste ALD30 mais nécessitant des soins continus d'une durée prévisible supérieure à 6 mois et une thérapeutique coûteuse) ;

- ALD32: polypathologies invalidantes nécessitant des soins continus d'une durée prévisible supérieure à 6 mois.

\section{Liste des 30 Affections Longue Durée (ALD 30) :}

La liste des Affections de Longue Durée a été modifiée par le décret n 2004 - 1049 du 4 Octobre 2004. (Journal Officiel. du 0510 2004)

- Accident vasculaire cérébral invalidant.

- Aplasie médullaire et autres cytopénies chroniques

- Artériopathies chroniques avec manifestations ischémiques.

- Bilharziose compliquée.

- Insuffisance cardiaque grave, troubles du rythme graves, cardiopathies valvulaires graves, cardiopathies congénitales graves

- Maladies chroniques actives du foie et cirrhoses.

- Déficit immunitaire primitif grave nécessitant un traitement prolongé, infection par le Virus de l'Immunodéficience Humaine (VIH).

- Diabète de type 1 et diabète de type 2

- Formes graves des affections neurologiques et musculaires (dont myopathie), épilepsie grave.

- Hémoglobinopathies, hémolyses, chroniques constitutionnelles et acquises sévères.

- Hémophilies et affections constitutionnelles de l'hémostase graves.

- Hypertension artérielle sévère.

- Infarctus coronaire.

- Insuffisance respiratoire chronique grave.

- Maladie d'Alzheimer et autres démences.

- Maladie de Parkinson.

- Maladies métaboliques héréditaires nécessitant un traitement prolongé spécialisé.

- Mucoviscidose.

- Néphropathie chronique grave et syndrome néphrotique primitif.

- Paraplégie.

- Périarthrite noueuse, lupus érythémateux aigu disséminé, sclérodermie généralisée évolutive.

- Polyarthrite rhumatoïde évolutive grave.

- Affections psychiatriques de longue durée.

- Rectocolite hémorragique et maladie de Crohn évolutives.

- Sclérose en plaques.

- Scoliose structurale évolutive (dont l'angle est égal ou supérieur à 25 degrés) jusqu'à maturation rachidienne.

- Spondylarthrite ankylosante grave

- Suites de transplantation d'organe

- Tuberculose active, lèpre.

- Tumeur maligne, affection maligne du tissu lymphatique ou hématopoiétique. 
ANNEXE II Les comportements face à l'annonce d'une pathologie chronique

\section{Acceptation ou résignation}

Le résultat de deux processus différents face à la maladie.

\section{ANNONCE DU DIAGNOSTIC}

\section{$\mathrm{CHOC}$ \\ (stupeur)}
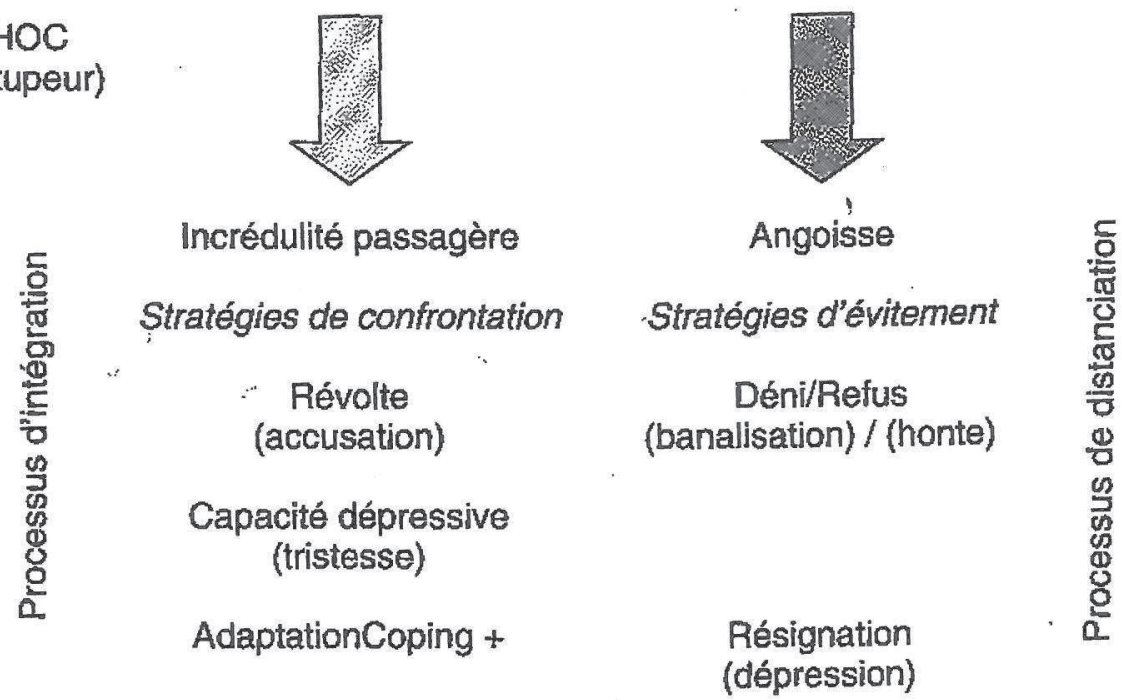

Travail du deuil inspiré de freud [1] 

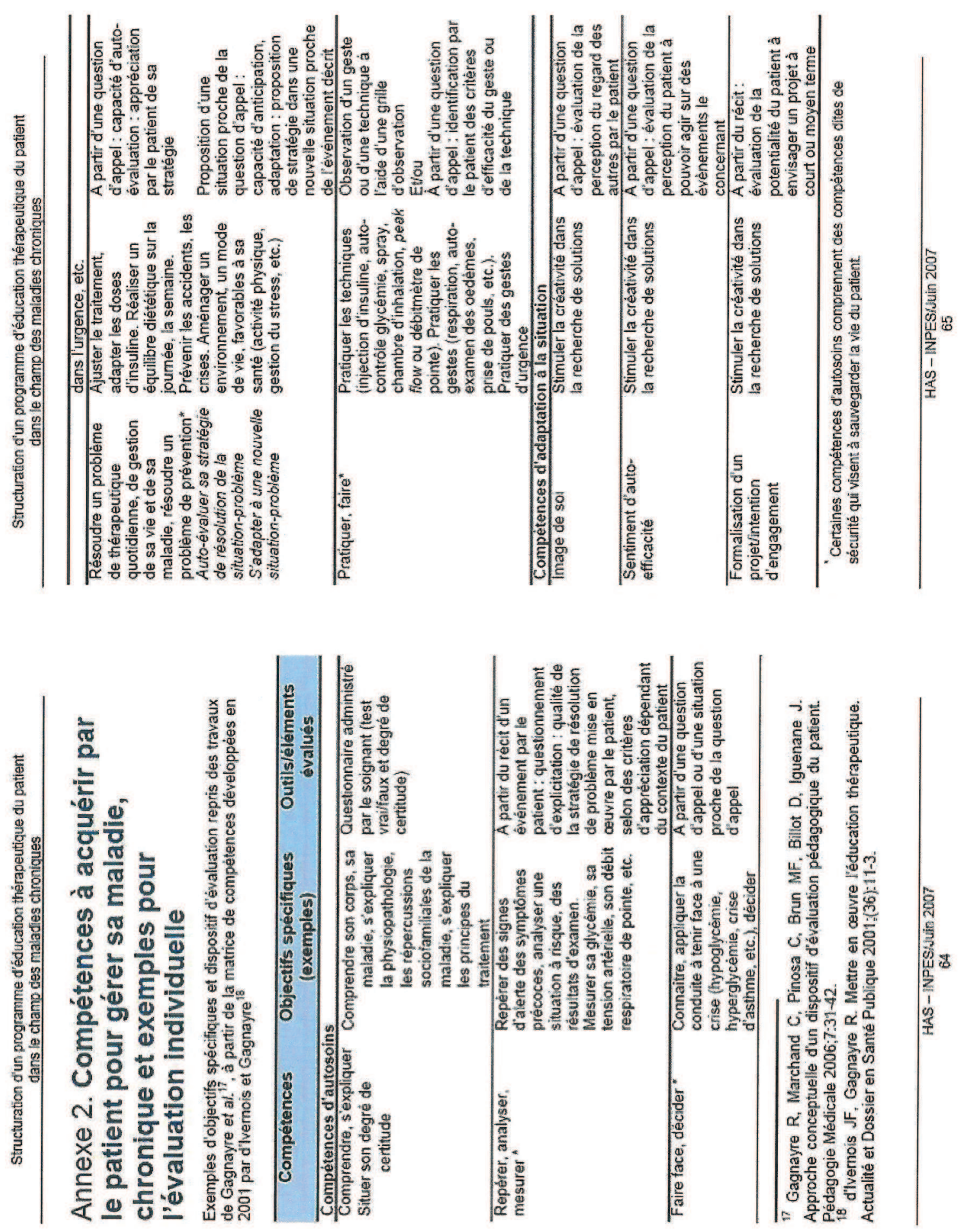


\section{ANNEXE IV Les crises}

\section{Crises épileptiques}

\section{crises partielles}

\begin{tabular}{|l|}
\hline \multicolumn{1}{|c|}{ simples } \\
\hline motrice (clonies dun membre ou doun hémicorps) \\
sensitive ( paresthésies) \\
sensorielle (visuelle, auditive, gustative) \\
végétative (pesanteur épigastrique ascendante) \\
psychique ( peur, impression de déjà w...) \\
dysphasique (vocalisation, mots, aphasie...) \\
sans altérations de la conscience
\end{tabular}

\begin{tabular}{|c|}
\hline complexes \\
\hline crises de types identiques \\
avec altérations de la conscience \\
et suspension d'activités ou activités \\
automatiques stéréotypées
\end{tabular}

\section{crises généralisées}

\section{Crise tonico-clonique $=$ crise grand $\mathrm{mal}$}

1 ère phase tonique :contraction intense

et généralisée de tous les muscles

apnés, cyanose

morsure de langue

2ème phaseclonique: secousses musculaires

brusques, généralisées, synchrones

3ème phase résolutive : coma post-critique

hypotonie généralisế, perte d'urines, reprise

de la respiration stertoreuse

confusion post-critique, amnésie

Crises myocloniques

secousses musculaires brères synchrones, bilatérales prédominant aux membres supérieurs et la face

sans perte de conscience

\section{les états de mal}

\begin{tabular}{|c|}
\hline État de mal conwulsif généralisé \\
\hline $\begin{array}{c}\text { crises tonico-cloniques avec } \\
\text { retentissements respiratoire et cérébral }\end{array}$ \\
\hline
\end{tabular}

État de mal partiel

Succession de crises partielles risque de généralisation

\begin{tabular}{|c|}
\hline État de mal partiel \\
\hline $\begin{array}{c}\text { Succession de crises partielles } \\
\text { risque de généralisation }\end{array}$ \\
\hline
\end{tabular}

\section{Absences= crise petit mal}

suspension brève de la conscience avec

internuption de factivité

souvent associée à des donies palpébrales crises récurrentes

sans intersalle de conscience normale ou crise unique prolongée

$>5 \mathrm{~min}$

\begin{tabular}{|c|}
\hline État de mal non conwulsif \\
\hline $\begin{array}{c}\text { absences, clonies discrètes, } \\
\text { troubles du comportement }\end{array}$ \\
\hline
\end{tabular}

Risques importants de sequelles dans tous les états de mal 
ANNEXE V Les antiépileptiques

Tableau 1. Conditions principales d'utilisation des traitements antléplleptlques

\begin{tabular}{|c|c|c|}
\hline Antiepileptique & $\begin{array}{c}\text { Indications } \\
\text { Posologie ( } \mathrm{P} \text { en mgikg) }\end{array}$ & $\begin{array}{c}\text { Risque d'aggravation de } \\
\text { certains syndromes } \\
\text { epileptiques }\end{array}$ \\
\hline Carbamazépine & $\begin{array}{l}\text { Épilepsies partielles ou } \\
\text { généralisées } \\
\text { c en monotherapie ou } \\
\text { en association s } \\
P=10-20\end{array}$ & $\begin{array}{l}\text { Spasmes infantiles, épilepsies } \\
\text { uvec crises myocloniques, } \\
\text { absences, POCS, abserices du } \\
\text { syndrome de Lennox-Gastaut, } \\
\text { crise tonique du syndrome de } \\
\text { Lennox-Gastaut. rares cas } \\
\text { d'aggravation des épilepsies } \\
\text { bennignes a pointes centro- } \\
\text { temporales }\end{array}$ \\
\hline Clobazam & $\begin{array}{l}\text { Tous types d'épilepsies } \\
\text { a en association s } \\
P=0.5-1\end{array}$ & \\
\hline clonazépam & $\begin{array}{l}\text { Tous types d'epilepsies } \\
\text { a en association ou en } \\
\text { monotheraple temporaire } y \\
P .=0.1-0.2\end{array}$ & \\
\hline Dlazépam & $\begin{array}{l}\text { Traitement d'urgence de retat } \\
\text { de mal } \\
\mathrm{P}=0.5 \mathrm{mg} / \mathrm{kg} \text {. total max. } 10 \mathrm{mg}\end{array}$ & \\
\hline Ethosuximide & $\begin{array}{l}\text { Epilepsie genéralisee (absence) } \\
\text { \& en monotherapte ou } \\
\text { en association s } \\
P=20-30\end{array}$ & \\
\hline Felbamate & $\begin{array}{l}\text { Syndrome de Lennox-Gastaut } \\
i \text { en association , } \\
P=15-45\end{array}$ & \\
\hline Fosphénytome & $\begin{array}{l}\text { Etat de mal } \\
\text { Substitution de la phénytoine } \\
\text { orale lorsque radministration } \\
\text { orale est impossible } \\
\text { ou contre-indiqueee }\end{array}$ & \\
\hline Gabapentine & $\begin{array}{l}\text { Epllepsies partielles } \\
\text { « en monothérapie ou } \\
\text { en association } \\
\mathrm{P}=20-35\end{array}$ & $\begin{array}{l}\text { Absences du syndrome de } \\
\text { Lennox-Gastaut } \\
\text { Clonies } \\
\text { Myoclonies }\end{array}$ \\
\hline Lamotrigine & $\begin{array}{l}\text { Épilepsies partielles ou } \\
\text { généralisées } \\
\text { Syndrome de L_ennox-Gastaut } \\
\text { « en monothérapie ou } \\
\text { en association \$ } \\
P=1-5 \text { (variable selon AE associé) }\end{array}$ & $\begin{array}{l}\text { Syndrome de Dravet } \\
\text { Éplepsie myoclonique }\end{array}$ \\
\hline Lévétiracétam & 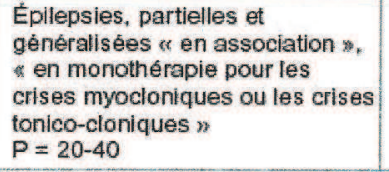 & \\
\hline Oxcarbazépine & $\begin{array}{l}\text { Epilepsies partielles } \\
\text { a en monothérapie ou } \\
\text { en association s } \\
\mathrm{P}=30-45\end{array}$ & \\
\hline Phenobarbital & $\begin{array}{l}\text { Epilepsles partielles ou } \\
\text { généralisées } \\
\text { État de mal (IV) } \\
\text { Convulsions néonatales } \\
\text { o en monothérapie : } \\
P=15-20 ; I V ; \text { nouveau-nés } \\
3-5<5 \text { ans ; } 2-3>5 \text { ans }\end{array}$ & $\begin{array}{l}\text { Spasmes infantlles; } \\
\text { Syndrome de Dravet } \\
\text { Pocs }\end{array}$ \\
\hline Phénytoine & $\begin{array}{l}\text { Epliepsles partielles ou } \\
\text { géneralisees } \\
\text { * en monothérapie ou } \\
\text { en association } s \\
P=15-20 ; \text {;V; nouveau-nés } \\
8-10<3 \text { ans; } 4-7>3 \text { ans }\end{array}$ & $\begin{array}{l}\text { POCS } \\
\text { Absences } \\
\text { Myocionies } \\
\text { myocionique progressive }\end{array}$ \\
\hline Prégabaline & $\begin{array}{l}\text { Épllepsies partielles avec ou } \\
\text { sans gênéralisation secondaire } \\
\text { ' en association } \\
\text { Indiqué chez l'adulte } \\
\mathrm{P}=150-600 \text { (mg/jour) }\end{array}$ & \\
\hline
\end{tabular}




\begin{tabular}{|c|c|c|}
\hline Primidone & $\begin{array}{l}\text { Épilepsles partielles et } \\
\text { gèneralisèes } \text { an monothérapie } \\
\text { ou en association } w \\
P=10-40\end{array}$ & \\
\hline Rufinamide & $\begin{array}{l}\text { Syndrome de Lennox-Gastaut } \\
\text { is en association i }\end{array}$ & \\
\hline Stiripentol & $\begin{array}{l}\text { Syndrome de Dravet } \\
\text { * en association au clobazépam } \\
\text { et à l'acide valproique " } \\
\text { indication pédiatrique } \\
\text { Autres indications, en ATU } \\
\text { nominative } \\
\mathrm{P}=50\end{array}$ & \\
\hline Sulthiame & $\begin{array}{l}\text { Epilepsie à paroxysmes } \\
\text { rolandiques } \\
\text { En ATU nominative } \\
\mathrm{P}=5-15\end{array}$ & \\
\hline Tiagabine & $\begin{array}{l}\text { Épilepsies partielles } \\
\text { "en association * } \\
P=0,5-2\end{array}$ & Absences \\
\hline Topiramate & $\begin{array}{l}\text { Épilepsies géneralisées } \\
\text { et partielles } \\
\text { Syndrome de Lennox-Gastaut } \\
\text { (hors AMM) } \\
\text { 4 en association ou en } \\
\text { monotherapie en deuxième } \\
\text { intention : } \\
\mathrm{P}=4-6\end{array}$ & \\
\hline $\begin{array}{l}\text { Valproate } \\
\text { de sodium }\end{array}$ & $\begin{array}{l}\text { Épilepsies partielles } \\
\text { ou généralisées } \\
\text { " en monotherapie ou } \\
\text { en association " } \\
P=15-40\end{array}$ & POCS \\
\hline Vigabatrine & $\begin{array}{l}\text { Spasmes infantlles } \\
\text { \& en monotherapie : } \\
\text { Epilepsies partielles } \text { « en } \\
\text { association en derniere intention } " \\
P=100-150: \text { spasmes infantiles } \\
P=20-80: \text { autres indications }\end{array}$ & $\begin{array}{l}\text { Épllepsies myocloniques } \\
\text { Absences }\end{array}$ \\
\hline Zonisamide & $\begin{array}{l}\text { Epilepsies partielles, épilepsies } \\
\text { myocloniques \& en association } n \\
\mathrm{P}=4-12\end{array}$ & \\
\hline
\end{tabular}

$\mathrm{AE}$ : antiépileptique.

W: intraveineux.

POCS : pointes-ondes ontinues du sommeil (aspect de l'EEG) ; celles-ci cowrent plus de 80 os du sommeil lent et se rencontrent dans différentes ëpilepsies graves.

Depuis 1985, le nombre de nouveaux $\mathrm{AE}$ a considérablement augmenté. Les plus

Le vigabatrin (Sabril ?mise sur le marché en 1990), lamotrigine (Lamictal, 1996$)$, le topiramate (Epitomax $\$ 1996$ ), la gabapentine (Neurontin 1996), loxcarbazépine (Trileptal 2000 ), le lévétiracétam (Keppra $\stackrel{3}{3} 2003$ ), le zonizamide (Zonegran 2008). 


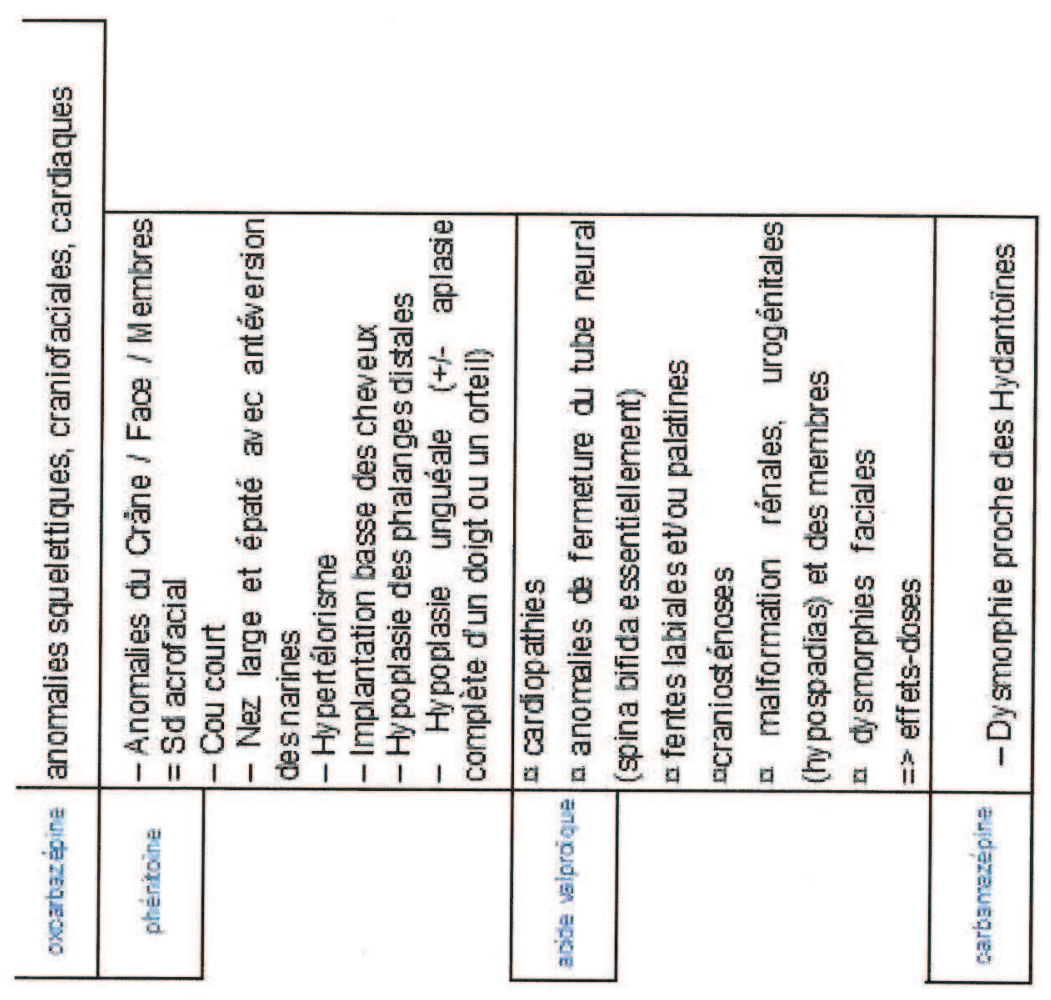

\begin{tabular}{|c|c|c|c|c|c|c|c|c|c|c|c|}
\hline 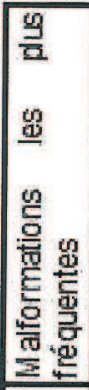 & 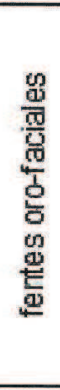 & 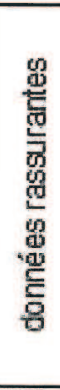 & & & & 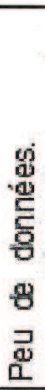 & & 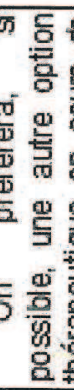 & & & \\
\hline 领 & $\begin{array}{l}\text { 흠 } \\
\text { 흘 } \\
\text { 듬 }\end{array}$ & 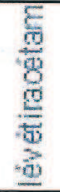 & 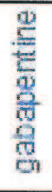 & 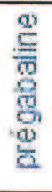 & 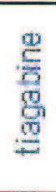 & 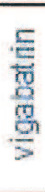 & 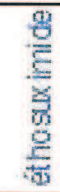 & 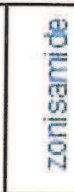 & 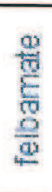 & 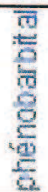 & 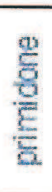 \\
\hline
\end{tabular}



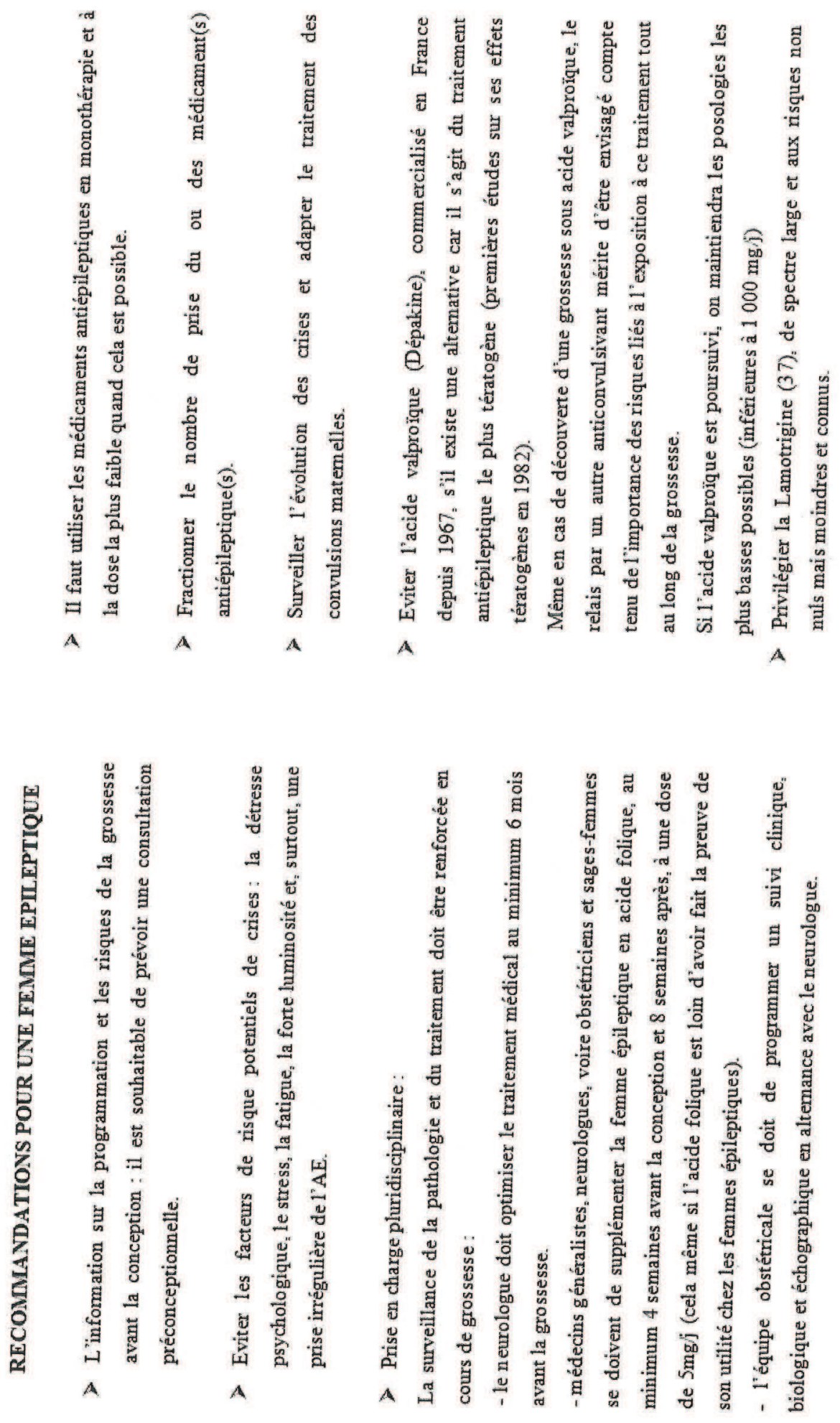
(suite)
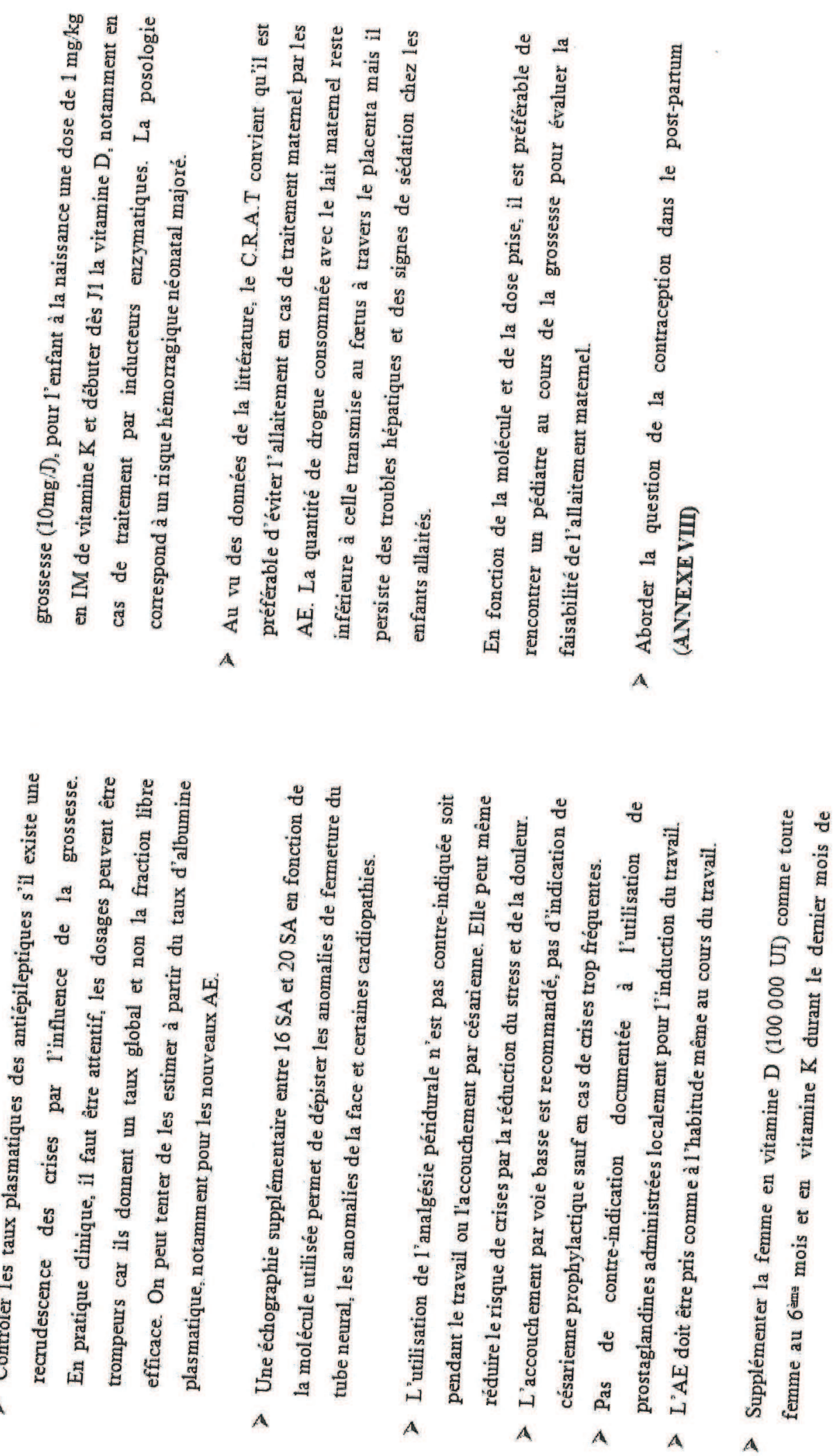


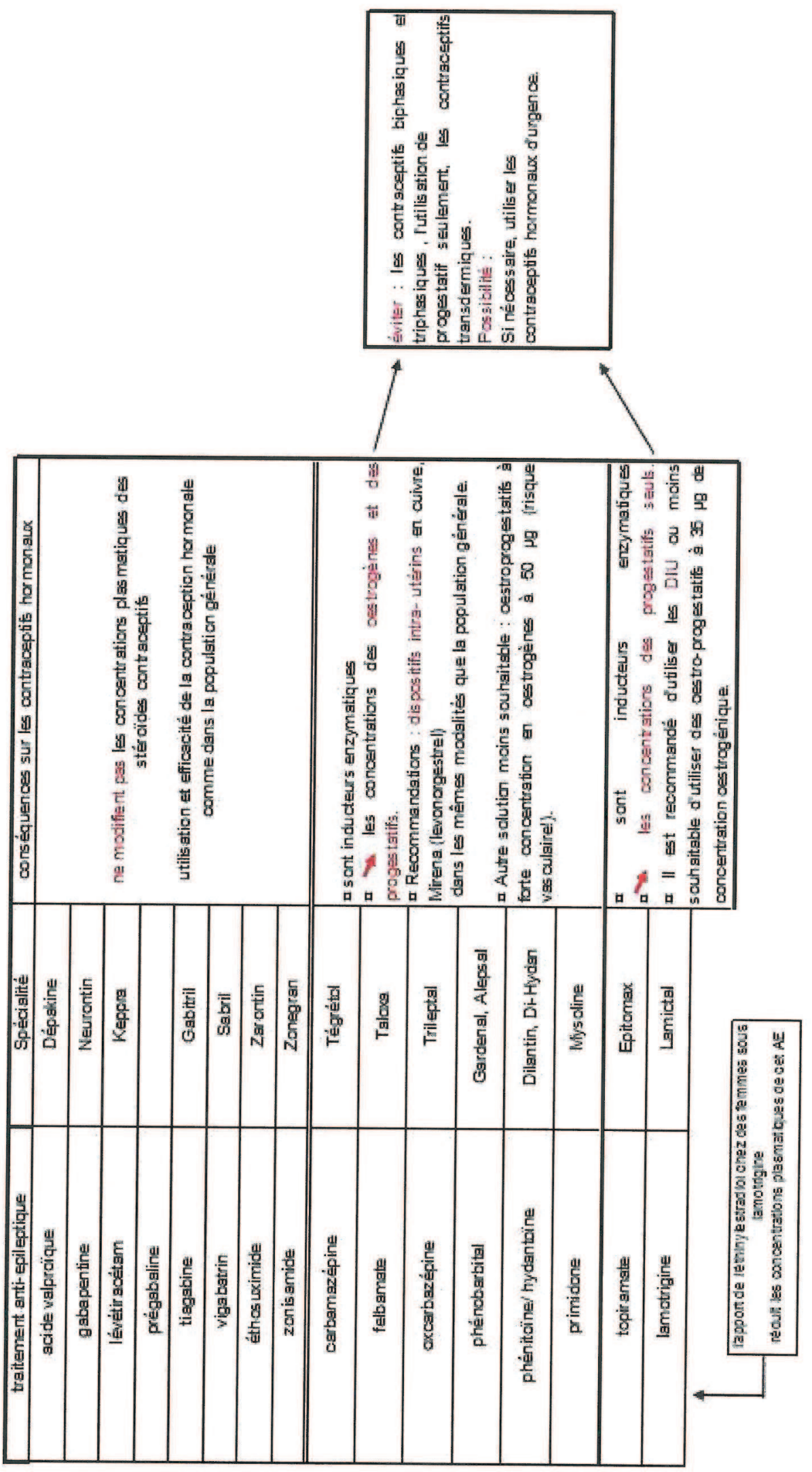




\section{ANNEXE IX Conduite à tenir en cas de crise chez la femme enceinte}

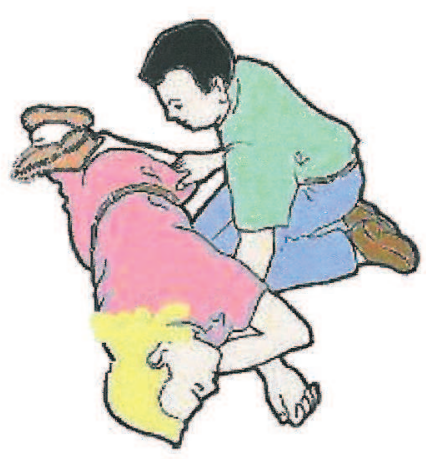

Devant une crise $=$ -Alerter

-Eviter les trauma : PLS, éloigner les objets dangereux, protéger -Eviter l'asphyxie : Canule de Guedel, O2, scope

-Si possibilité, écouter les bruits du coeur foetal

-Chronométrer pour reconnaître un êtat de mal

-Dextro (I'hypoglycêmie pouvant être une cause de crise)

-VAV : si crise $>1 \mathrm{~min}$ : prêparer 1 ampoule de Rivotril,

si crise $>\mathbf{5} \mathbf{m i n}:$ injecter en IV sur $1 \mathrm{~min}$

autres anti-convulsants utilisés : Valium $@$ en $\Gamma$ V

Si la crise a été provoquée :

-Trouver et traiter le facteur déclenchant

-Pas de traitement antiépileptique nécessaire a priori 


\section{ANNEXE X Questionnaire}

\section{Questionnaire}

Madame (nom de la sage-femme) ou Monsieur (nom),

Etudiante sage-femme en dernière année à l'école de Rouen, je réalise, dans ce cadre, mon mémoire de fin d'études.

En tant que future professionnelle, j'ai choisi d'évaluer la place de la sage-femme dans la prise en charge des patientes atteintes de pathologies chroniques.

Ainsi, je me permets de tester certaines de vos connaissances, les moyens à votre disposition et votre expérience de sage-femme face aux pathologies chroniques et notamment à un cas qui m’interpelle davantage : les patientes épileptiques.

Ce travail est basé sur un questionnaire. Je me permets de vous solliciter quelques minutes.

Il sera anonyme. Après avoir retiré cette première page nominative, vous pourrez le déposer dans l'urne qui se trouve dans votre unité d'exercice.

Je sais que votre emploi du temps est bien chargé, je vous remercie d'avance pour votre intérêt et du temps que vous m'accorderez.

Adeline VOISIN 

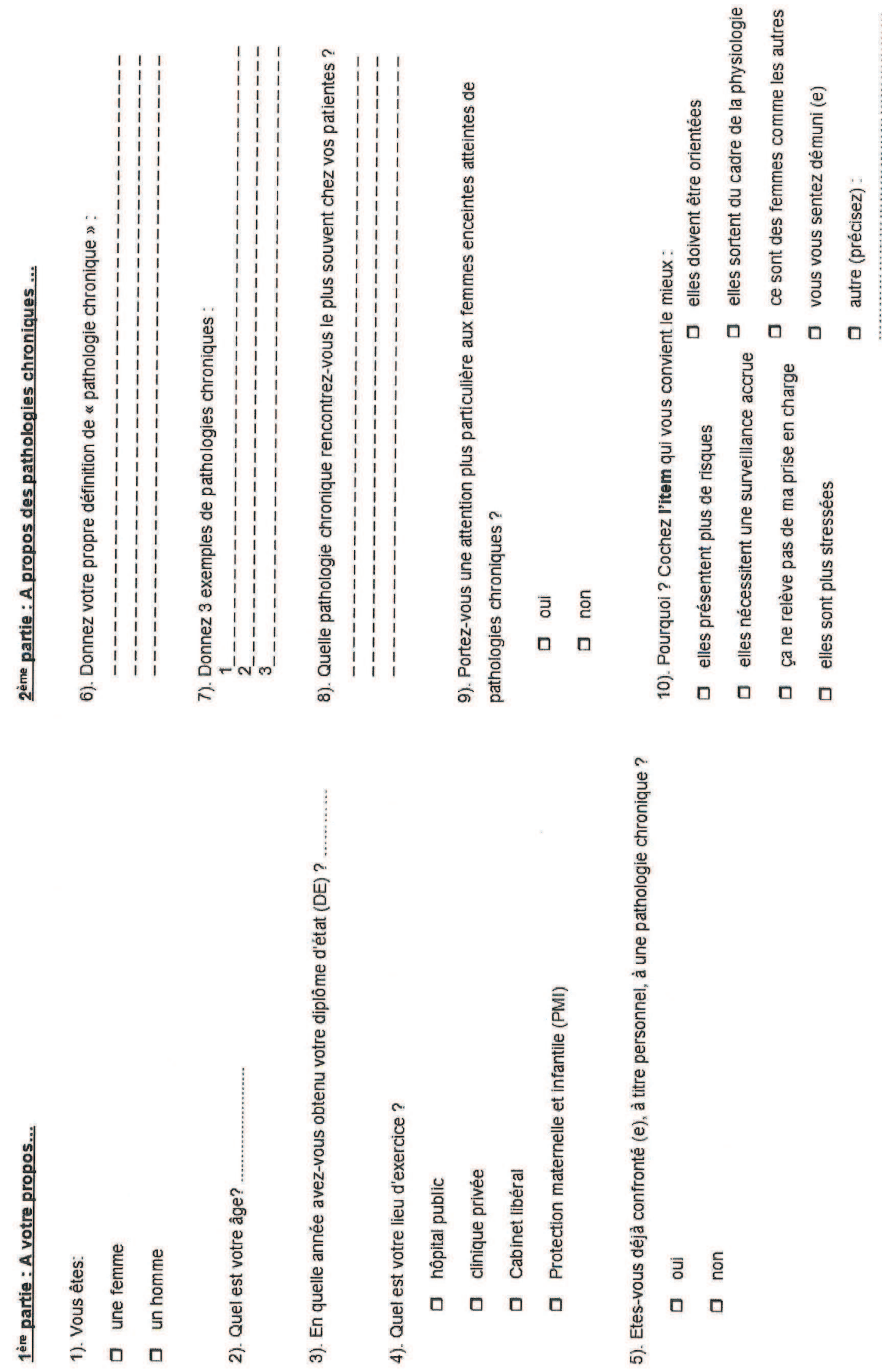

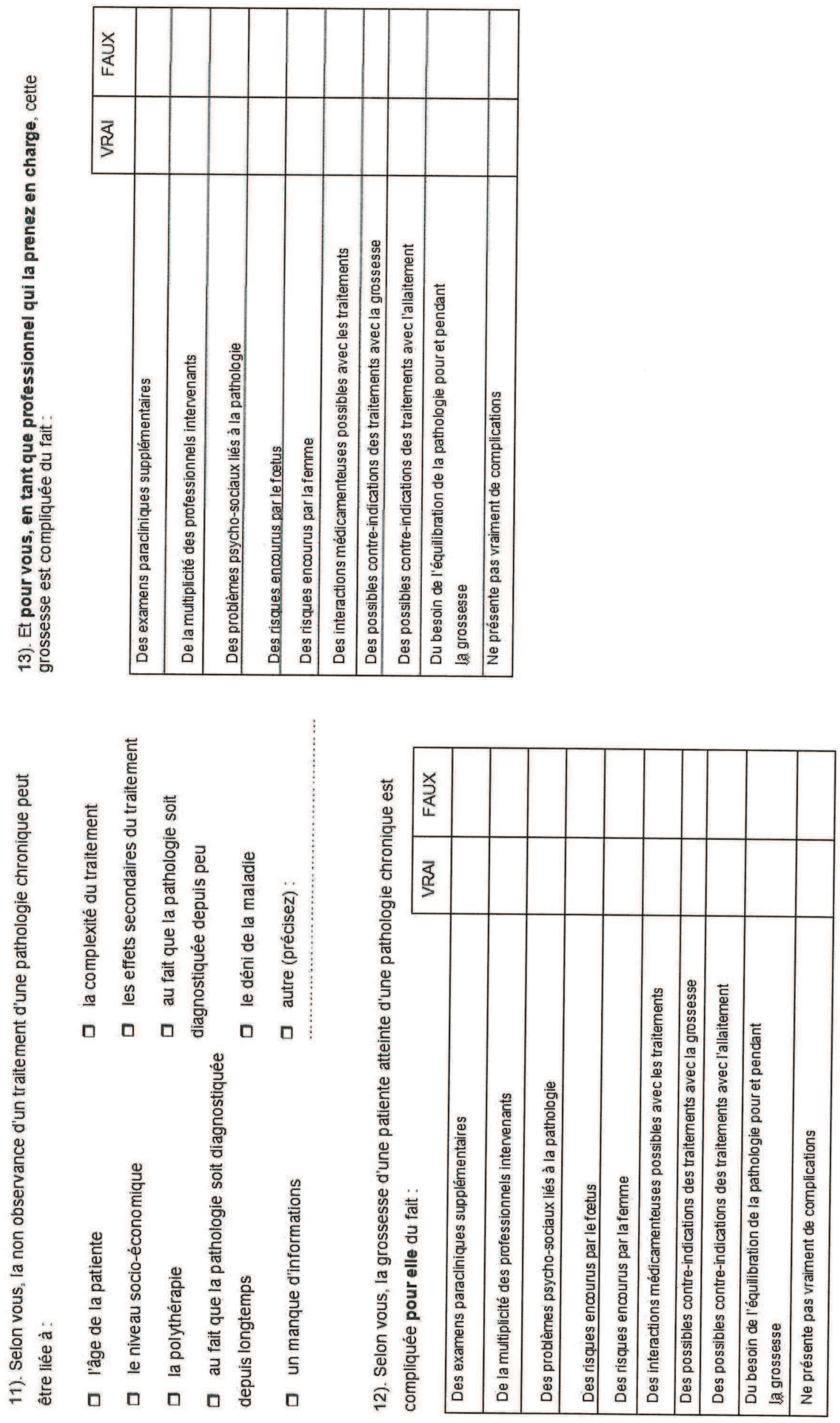

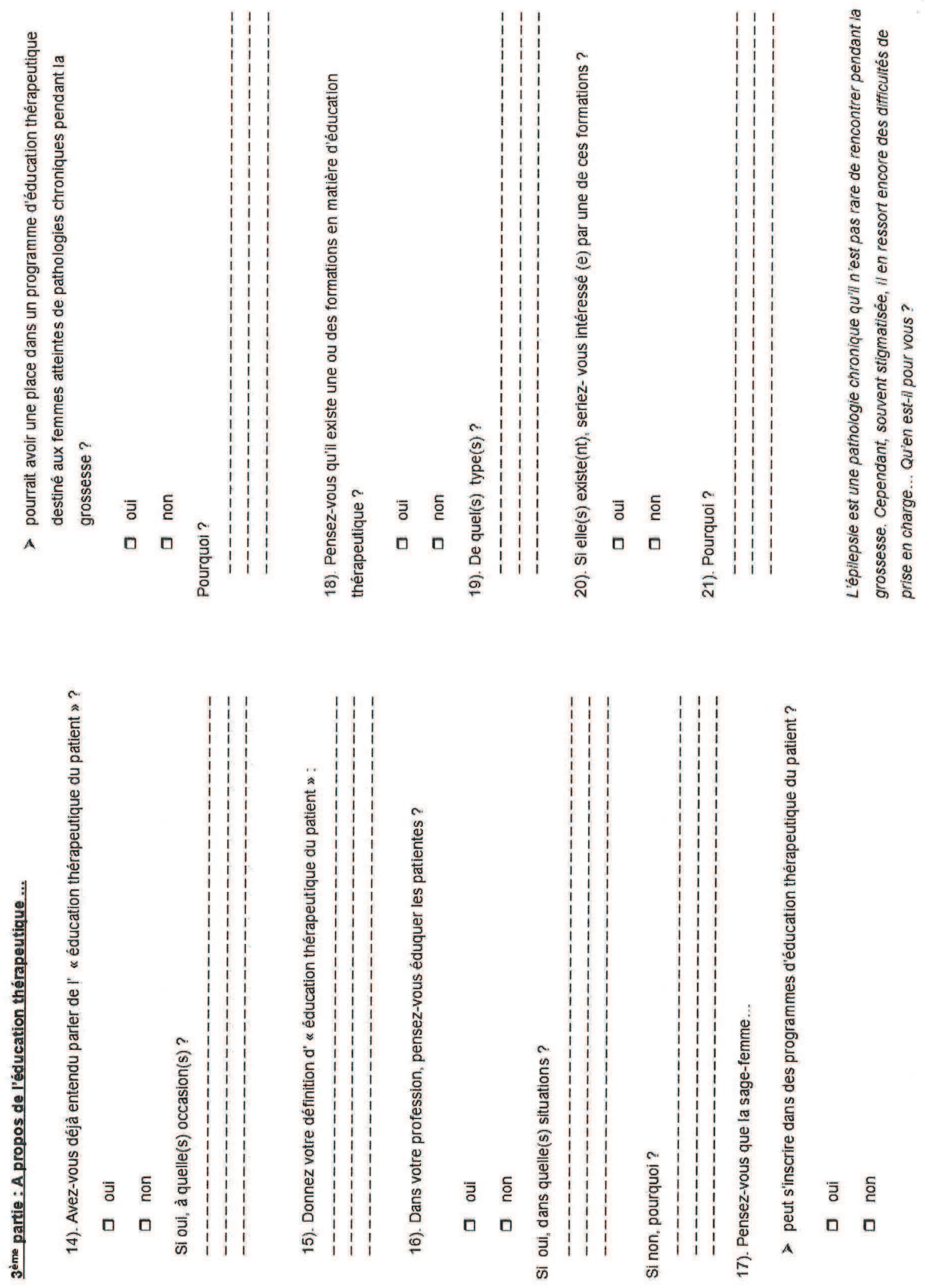

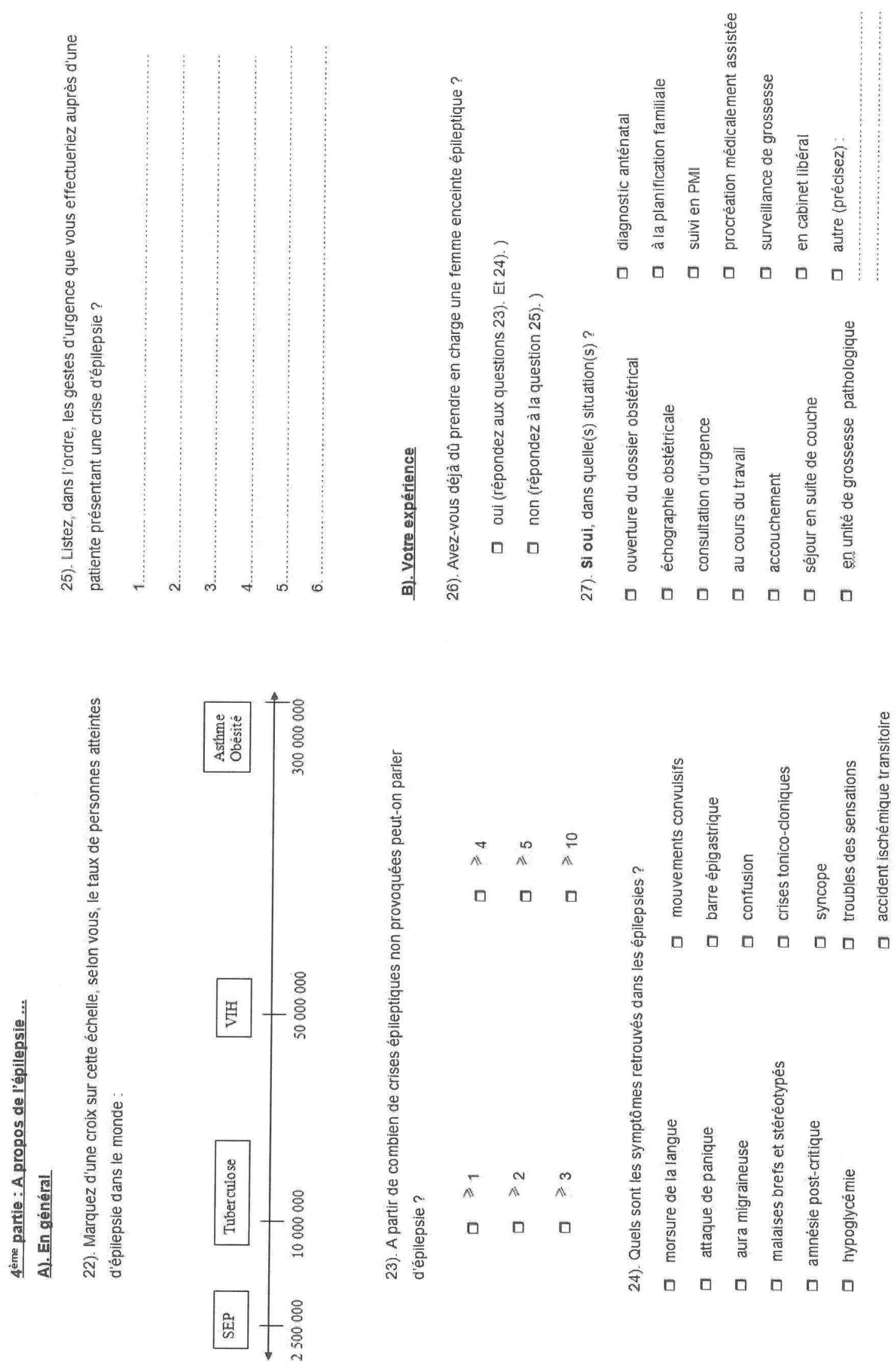

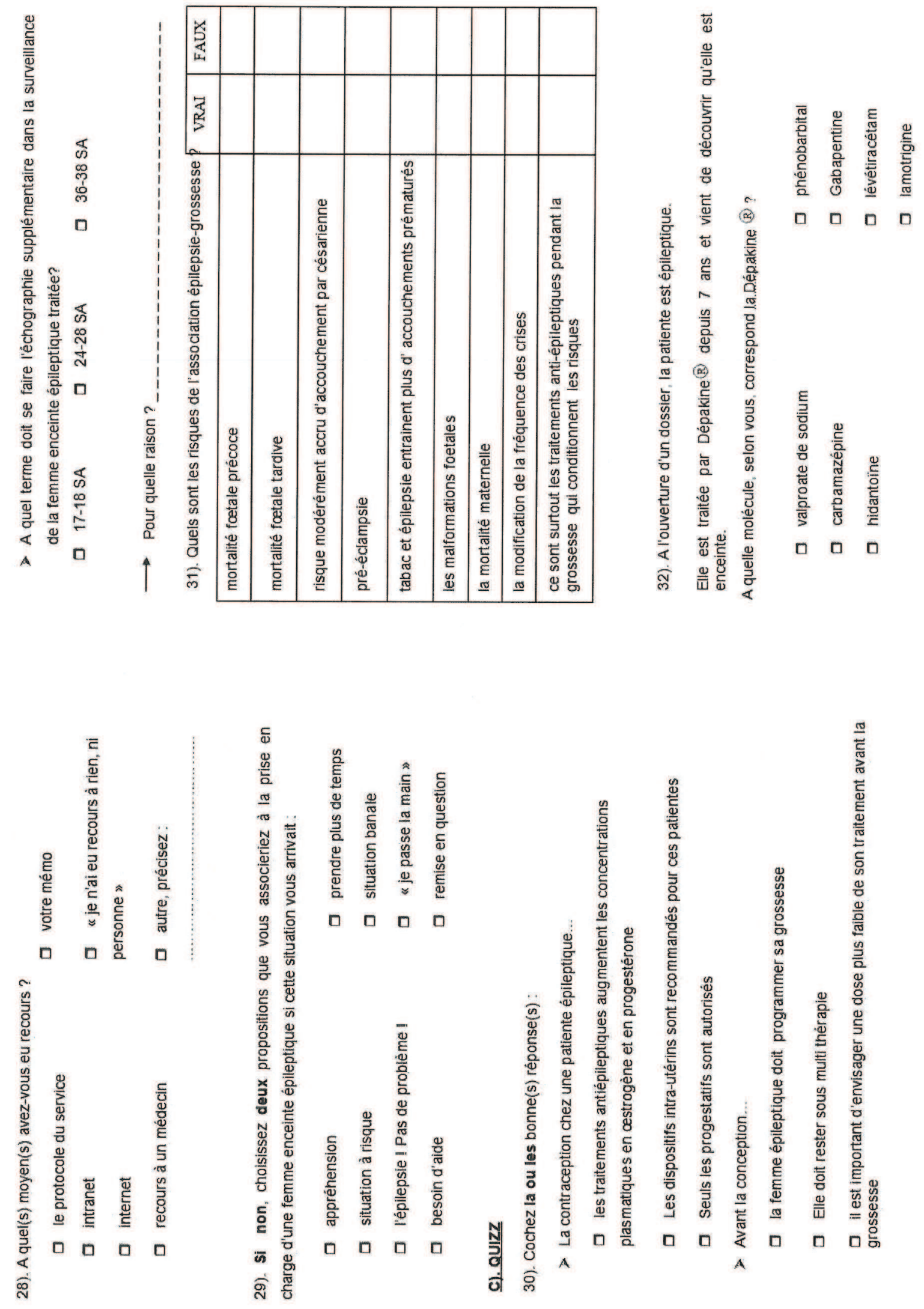

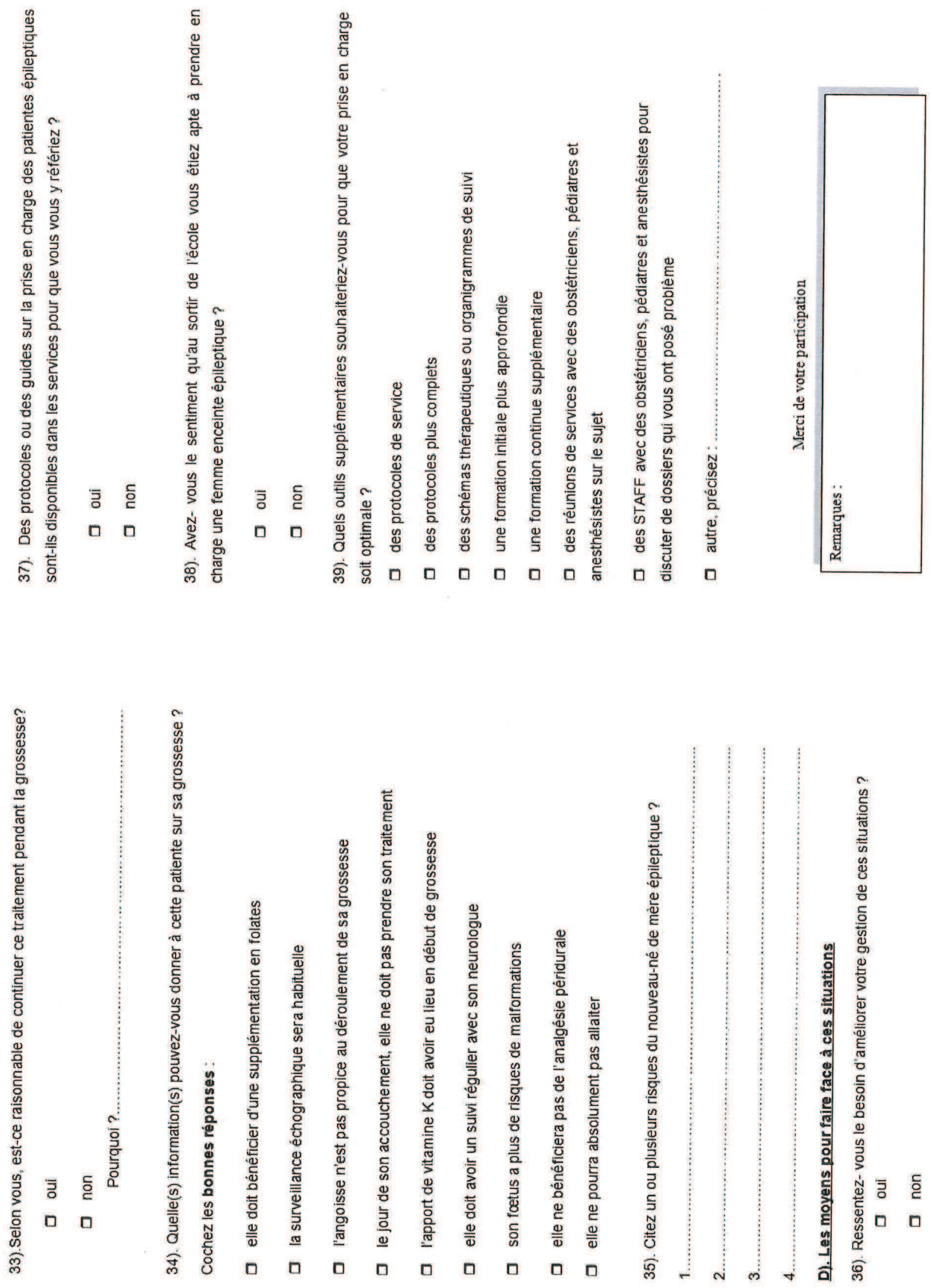


\section{Recommandations de l'HAS 2007 sur l'orientation des femmes enceintes}

Types de suivi recommandés

- Suivi A: selon le choix de la femme, le suivi régulier peut être assuré par une sage-femme ou un médecin (généraliste, gynécologue médical ou gynécologue-obstétricien).

- Avis A1 : l'avis d'un gynécologue-obstétricien et' ou d'un autre spécialiste est conseillé.

- Avis A2 : l'avis d'un gynécologue-obstétricien est nécessaire. L'avis complémentaire d'un autre spécialiste peut également être nécessaire.

- Suivi B : le suivi régulier doit être assuré par un gynécologue-obstétricien.

\section{Types de structures requises}

- Unité d'obstétrique : telle que définie dans l'article R. 6123-43 du Code de la santé publique « une unité dobstétrique assure, tous les jours de lannée, vingt-quatre heures sur vingt-quatre, les accouchements ainsi que les actes de chirurgie abdomino-pelvienne liés à la grossesse, à laccouchement et à la délvrance. Elle participe également au dépistage des facteurs de risques durant la grossesse, notamment dans le cadre des consultations prénatales. Elle assure les soins suivant laccouchement pour la mère et les enfants nouveaunés dont la naissance est intervenue dans l'établissement. Si létablissement dans lequel est né l'enfant ne peut assurer sa prise en charge adaptée, il organise son transfert vers un autre établissement apte à déliver les soins appropriés. "

- $\dot{A}$ adapter en fonction du risque pour la mère et l'enfant à naître.

Période préćconceptionnelle ou $1^{\text {th }}$ consultation de grossesse Situations à risque Suivi Structure

Antécédents médicaux et pathologies préexistantes non gynécologiques Usage de drogues illicites (héroine, ecstasy, cocaine sauf cannabis) et substituts (méthadone) et sevrage

Hypertension artérielle

Diabètes

Thrombose veineuse profonde-embolie pulmonaire

- Affections thyroidiennes équilibrées (selon la pathologie) hors maladie de Basedow

- Maladie de Basedow

Epilepsie traitée ou non traitée

- Asthme ou pathologie pulmonaire (avec incidence sur la fonction respiratoire)

- Asthme modéré, ancien, contrôlé

Maladies inflammatoires digestives

(maladie de Crohn ou rectocolite hémorragique traitée)

Drépanocytose et autres hémoglobinopathies :

- mère homozygote

- mère hétërozygote

Anomalies de la coagulation, thrombopénie maternelle

et purpura thrombopénique auto-immun

Maladies de système et maladies rares (sclérose en plaques,

Addison, Cushing, LED, SAPL, sclérodermie, polyarthrite rhumatoìde)

- Néphropathie

- Uropathie

Affection hépatique (cholestase, maladie biliaire, adénome)

Chimiothérapie et radiothérapie

Maladies psychiatriques sévères (pathologie elle-même et facteurs de risque liés à la

thérapeutique) : schizophrénie, troubles bipolaires, syndromes dépressifs sévères

\begin{tabular}{|c|c|}
\hline Suivi & Structure \\
\hline A1 & $\begin{array}{l}\text { Unité d'cbstétrique si antécédent } \\
\text { ancien }\end{array}$ \\
\hline B & A adapter \\
\hline B & A adapter \\
\hline B & $A$ adapter \\
\hline A1 & Unité d'obstétrique \\
\hline B & A adapter \\
\hline A2 précoce & A adapter \\
\hline B & A adapter \\
\hline A & Unitè d'obstétrique \\
\hline A2 précoce & A adapter \\
\hline B & $\begin{array}{l}\text { À adapter } \\
\text { Unité d'ohstétrique }\end{array}$ \\
\hline A2 & \\
\hline B & A adapter \\
\hline B & A adapter \\
\hline $\begin{array}{c}\mathrm{B} \\
\mathrm{A} 2\end{array}$ & $\begin{array}{l}\text { Á adapter } \\
\text { Unité d'obstétrique si sans } \\
\text { retentissement }\end{array}$ \\
\hline A2 précoce & A adapter \\
\hline A2 & Unité d'obstétrique \\
\hline A2 précoce & A adapter \\
\hline
\end{tabular}


ANNEXE XII Exemple de schéma thérapeutique

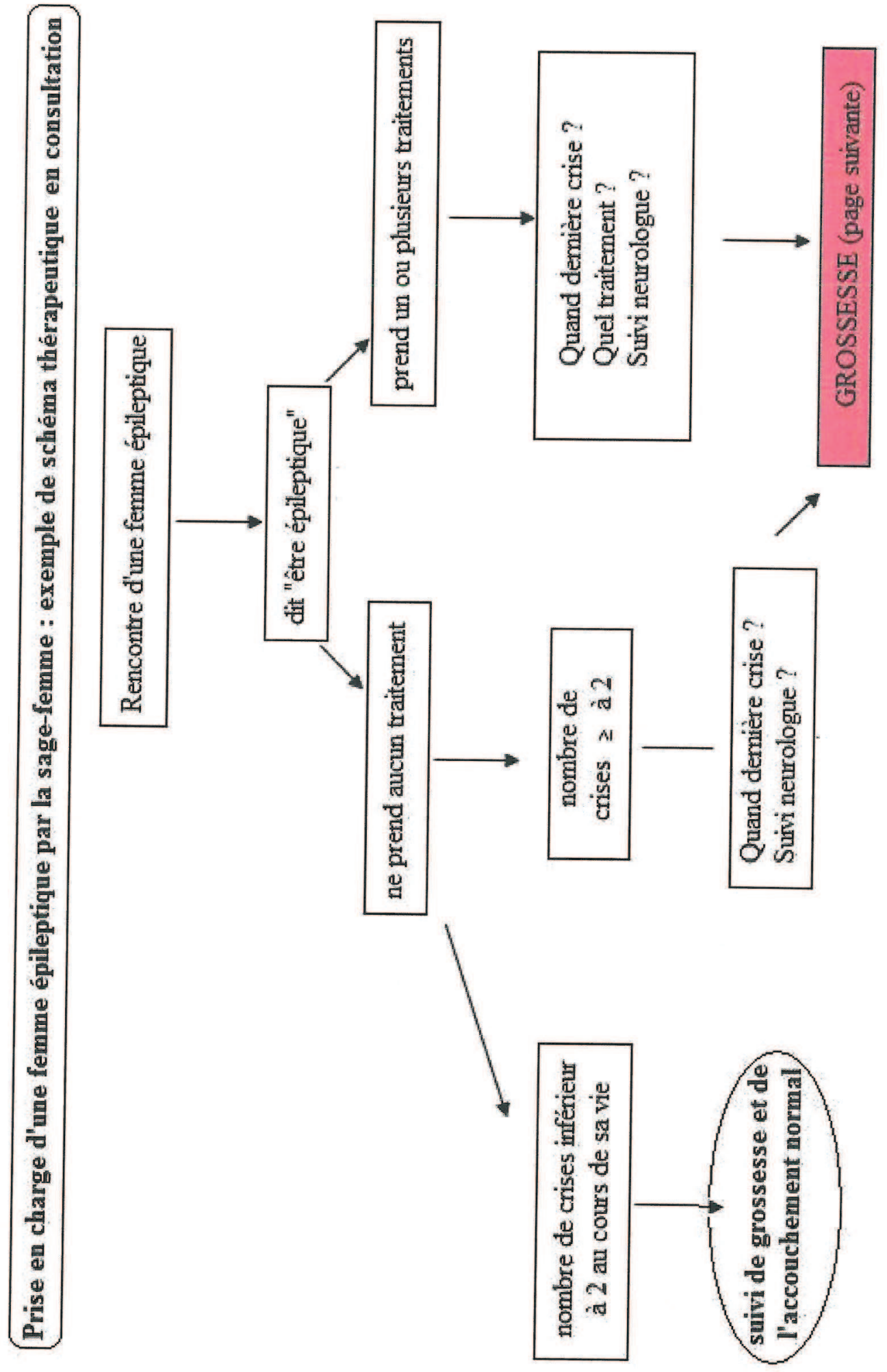




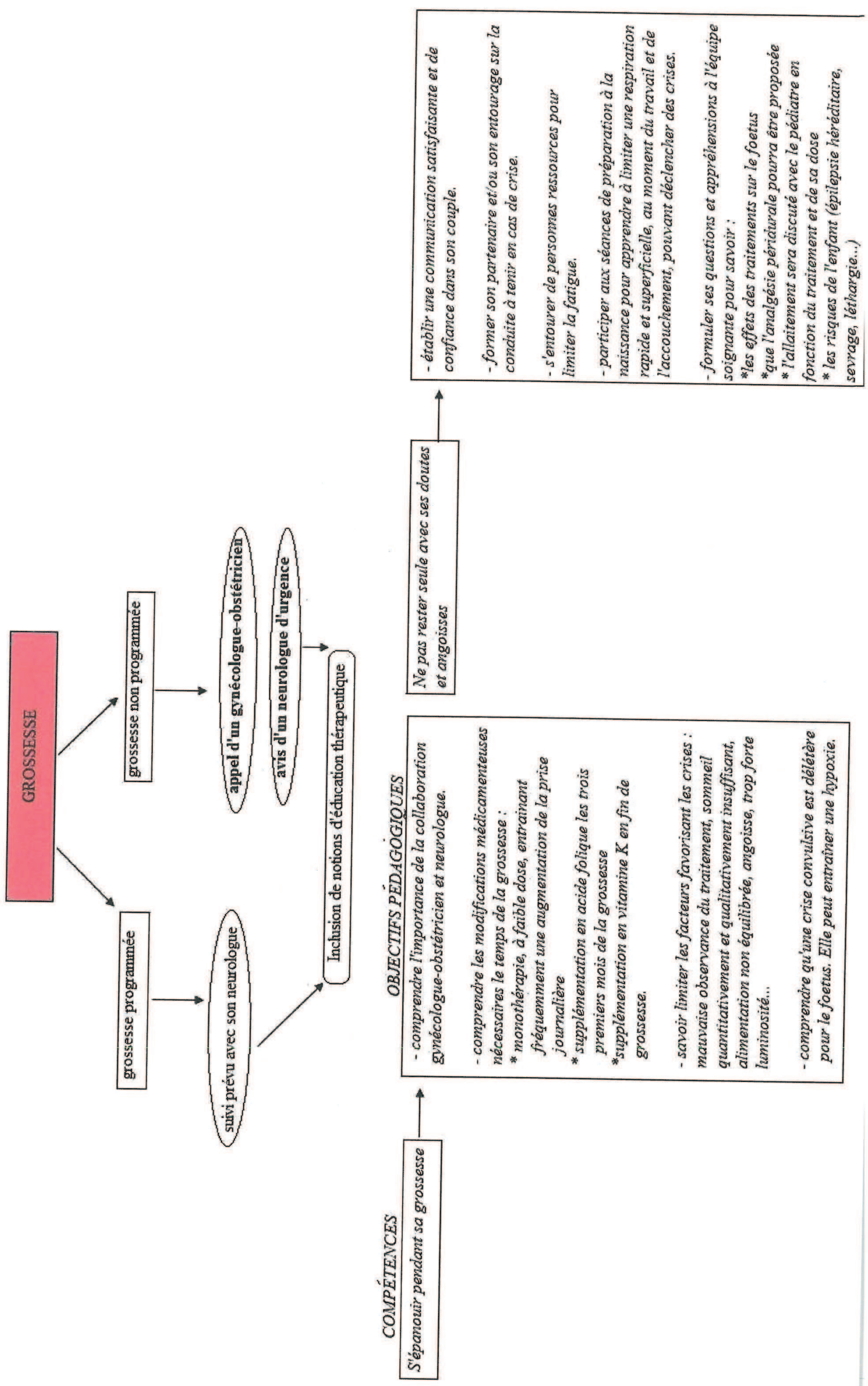

